\title{
Detachable Pairs in \\ 3-Connected Matroids
}

\author{
Alan Williams
}

A thesis

submitted to Victoria University of Wellington

in fulfilment of the requirements for the degree of

Doctor of Philosophy

in Mathematics.

Victoria University of Wellington

2015 


\begin{abstract}
The classical tool at the matroid theorist's disposal when dealing with the common problem of wanting to remove a single element from a 3-connected matroid without losing 3-connectivity is Tutte's Wheels-and-Whirls Theorem. However, situations arise where one wishes to delete or contract a pair of elements from a 3-connected matroid whilst maintaining 3-connectedness. The goal of this research was to provide a new tool for making such arguments. Let $M$ be a 3 -connected matroid. A detachable pair in $M$ is a pair $x, y \in E(M)$ such that either $M \backslash x, y$ or $M / x, y$ is 3-connected. Naturally, our aim was to find the necessary conditions on $M$ which guarantee the existence of a detachable pair. Triangles and triads are an obvious barrier to overcome, and can be done so by allowing the use of a $\Delta-Y$ exchange. Apart from these matroids with three-element 3-separating sets, the only other class of matroids that fail to contain a detachable pair for which no bound can be placed on the size of the ground set is the class of spikes. In particular, we prove the following result. Let $M$ be a 3 -connected matroid with at least thirteen elements. If $M$ is not a spike, then either $M$ contains a detachable pair, or there exists a matroid $M^{\prime}$ where $M^{\prime}$ is obtained by performing a single $\Delta-Y$ exchange on either $M$ or $M^{*}$ such that $M^{\prime}$ contains a detachable pair. As well as being an important theorem in its own right, we anticipate that this result will be essential in future attempts to extend Seymour's Splitter Theorem in a comparable manner; where the goal would be to obtain a detachable pair as well as maintaining a 3-connected minor. As such, much work has been done herein to study the precise configurations that arise in 3-separating subsets which themselves yield no detachable pair.
\end{abstract}




\section{Acknowledgements}

Thanks first and foremost to Geoff Whittle.

Thank you also to Carolyn Chun, Peter Donelan, Richard, and Mary. 



\section{Contents}

1 Introduction 1

1.1 Definitions . . . . . . . . . . . . . . . . 6

1.2 Further Preliminary Lemmas . . . . . . . . . . . . . . . . 13

2 Obstacles 21

2.1 Triangles . . . . . . . . . . . . . . . . . . . . . . . 21

2.25 -Point Planes . . . . . . . . . . . . . . . . . . . 25

2.3 Specific Triad Situations . . . . . . . . . . . . . . 31

2.4 Flans . . . . . . . . . . . . . . . . . . 36

3 Global Structure in a Minor 43

3.1 Sequential Minor . . . . . . . . . . . . . . . . . . . 43

3.2 Internally 4 -Connected Minor . . . . . . . . . . . 50

4 Troublesome 3-Separating Sets $\quad 59$

4.1 To a More Structured 3-Separating Set . . . . . . . . . . . . . 59

4.2 The Triad Case . . . . . . . . . . . . . . . . . . . 63

4.3 The Non-Triad Case . . . . . . . . . . . . . . . . 85 
CONTENTS

5 Putting the Pieces Together $\quad 97$

5.1 Further Lemmas . . . . . . . . . . . . . . . . . . . . 97

5.2 Proof of Main Theorem . . . . . . . . . . . . . . . . . 100 


\section{Chapter 1}

\section{Introduction}

Matroids were originally formalised [21] in an attempt to generalise the concept of independence that occurs in several branches of mathematics; in particular, the concepts of linear independence in vector spaces, and edge sets of forests in graph theory. It quickly became clear however that the notion of independence captured in the definition was far more general than any one area for which it was first intended. There exist matroids which cannot be constructed from graphs; taking any field $\mathbb{F}$, there exist matroids which cannot be constructed from a finite collection of vectors in $\mathbb{F}$; and, indeed, there exist matroids which cannot be constructed from any field whatsoever (see [20]). A matroid which can be constructed from a vector space over some field $\mathbb{F}$ is said to be representable over $\mathbb{F}$, and certainly, a given matroid may be representable over one field but not another. Whether or not it is possible to represent a matroid over a particular field is a matter which is essentially decided by two closely related concepts. The underlying geometry of the vector space over the field and the degree of freedom the space allows in accepting new points, together with the pigeonholing of the raw combinatorial possibilities that arise from the matroidal definition.

Thus, since its inception, one of the primary areas of research in matroid theory has been in attempting to understand this distinction between 
representable and non-representable matroids. With the class of matroids representable over a particular field being closed under taking minors, the predominant approach has been in aiming to classify these various classes according to some list of excluded minors. We know from Tutte [18] that there exists exactly one excluded minor for the class of matroids which are representable over the two-element field; in particular, a matroid is representable over GF(2) if and only if it has no minor isomorphic to $U_{2,4}$. Next, Bixby [2] and Seymour [16] proved independently that the class of GF(3)-representable matroids has exactly three excluded minors. The complete characterisation for GF(4) in terms of excluded minors was then given by Geelen, Gerards, and Kapoor [5] in 2000 with seven such matroids. To date, the four-element field remains the largest field for which such an explicit characterisation has been arrived at.

In stark contrast to these encouraging results for small fields, such a concise classification has been shown to be not possible over the real numbers. Indeed, letting $\Gamma$ be the class of all real-representable matroids, Mayhew, Whittle, and Newman [9] have shown that for any real-representable matroid $M$, there exists an excluded minor for $\Gamma$ which itself has $M$ as a minor. However, in the case of finite fields, it has long been suspected that a finite classification will always be possible. Formally, Rota [15] conjectured that for any finite field $\mathbb{F}$, there exists only finitely many excluded minors for the class of $\mathbb{F}$-representable matroids. Indeed, it has recently been announced [6] that Rota's Conjecture has been resolved in the affirmative as a consequence of the work done in the matroid minor structure theorems of Geelen, Gerards, and Whittle.

So, whilst Rota's conjecture has been resolved, the solution we have is very much one of a qualitative, existential nature. The task of explicitly finding and listing the particular excluded minors for some given field is essentially another matter altogether. Obtaining such precise classifications has proven to be difficult to say the least for all but the most simple of fields. The GF(2) case is trivial, the GF(3) straightforward enough, the GF(4) man- 
ageable, and anything larger; as we have mentioned, remains out of our reach at this time due to the explosion in complexity as more allowable structure is able to be captured. One aspect contributing to this lies in the fact that for larger fields, a given matroid may have many different representations which are inequivalent in the natural algebraic sense. The fact that this phenomenon does not occur in the very smallest of fields simplifies matters greatly. In particular, all matroids representable over $\mathrm{GF}(2)$ and $\mathrm{GF}(3)$ are uniquely representable in the sense that every representation is algebraically equivalent, so that this issue is a non-issue. Over GF(4), the problem with the existence of inequivalent representations starts to arise. However, matroids representable over $\mathrm{GF}(4)$ are uniquely representable provided they are 3 -connected and we fix a representation of a $U_{2,4}$ minor [7]. The matroid $U_{2,4}$ is said to be a stabilizer [22] for the class of $\mathrm{GF}(4)$-representable matroids in this sense. The classification of the excluded minors for GF(4) relies crucially on this notion of a stabilizer, and it is hoped that it will also be of use in future attempts at classifications of larger fields. As the underlying field is enlarged, the problem with inequivalent representations only worsens. Oxley, Vertigan, and Whittle [13] showed that 3-connected GF(5)-representable matroids can have up to six inequivalent representations, and that, for any larger field, no such bound exists. Nevertheless, it is hoped that by generalising the techniques implemented in the GF(4) case [5], we may be able to gain significant traction on the class of matroids representable over GF(5), as well as the class of 2-regular matroids and the closely related class of matroids representable over all fields $\mathbb{F}$ for which $|\mathbb{F}| \geq 4$.

A key component of the work done in [5] lies in analysing when one can remove elements from a 3 -connected matroid which has a $U_{2,4}$ minor without losing 3-connectivity whilst simultaneously maintaining a copy of the minor. Essentially this boils down to the use of Seymour's Splitter Theorem [17], together with some consequences of it in order to bound the size of a potential excluded minor. Recall that the classical splitter theorem states that provided our original matroid is not a wheel or a whirl, we are always 
guaranteed the existence of a single element which is either deletable or contractable whilst maintaining 3-connectivity, and, moreover, after doing so will leave us with a matroid which preserves the property of containing a particular 3-connected minor of interest. While the splitter theorem has proved invaluable, there has been growing desire in recent times to find a stronger analogue to help deal with situations in which we wish to delete a pair of elements, or contract a pair of elements, all the while keeping 3-connectivity and a particular 3-connected minor. In such a setting, the original splitter theorem provides no guarantees as it is only equipped to deal with single element removals. Indeed, if it turned out to be the case that we could always delete or contract a pair of elements in this way, it has been noted by Jim Geelen (private communication) that the proof of the excluded-minor characterization for $\mathrm{GF}(4)$ could be simplified greatly into a short succinct argument.

Moreover, in recent work of Clark [4], attempts have been made to generalise the techniques of [5] to arbitrary (partial) fields and stabilizers for those fields. Much of the work done therein requires that precisely such an aforementioned analogue of the splitter theorem be obtainable. In particular, he has shown that; modulo the acquisition of a 2-element splitter theorem, and up to $\Delta-Y$ equivalence, an excluded minor for the class of $\mathbb{P}$-representable matroids has its size bounded by the size of a stabilizer $N$ for that class, or else is a two-element extension or coextension of an $N$-fragile matroid.

This is certainly an encouraging result, however the splitter theorem required for it to hold remains a conjecture at this stage. Thus, the search for such a theorem is certainly well motivated, and was one of our primary reasons for pursuing the line of enquiry contained in this work. Indeed, initially in this research project, we attempted to tackle the splitter problem directly; navigating all potential counterexamples that arose at once. After some time, it became clear that such a brute force approach was not gaining a huge amount of traction and was making it difficult to isolate and study the key problematic structures that arise. We decided that the best way to 
proceed, and, in retrospect, the most natural way to proceed, was to split the problem into its two essential components. First, there was the issue of finding a two-element analogue of Tutte's Wheels-and-Whirls Theorem [19]. In particular, we wanted to know when it was possible to either delete a pair of elements from a 3-connected matroid whilst maintaining 3-connectivity, or contract a pair of elements from a 3-connected matroid whilst maintaining 3 -connectivity. From there, the goal would be to use what was learnt to extend this result to a splitter theorem analogue with the added requirement of maintaining a 3-connected minor.

Our efforts from then on were primarily concentrated on completing such an appropriate analogue of the Wheels-and-Whirls Theorem. If $M$ is a 3connected matroid with $x, y \in E(M)$, we say that $\{x, y\}$ is a detachable pair if either $M \backslash x, y$ or $M / x, y$ is 3 -connected. The main result of this thesis is the following:

Theorem 1.0.1. Let $M$ be a 3-connected matroid with $|E(M)|>12$. If $M$ is not a spike, then either $M$ contains a detachable pair, or there exists a matroid $M^{\prime}$ where $M^{\prime}$ is obtained by performing a single $\Delta-Y$ exchange on either $M$ or $M^{*}$ such that $M^{\prime}$ contains a detachable pair.

Note that allowing ourselves use of the $\Delta-Y$ operation is perfectly safe in the sense that if $M$ is an excluded minor for a class of matroids representable over a partial field $\mathbb{P}$, and $M^{\prime}$ is obtained from $M$ via a $\Delta-Y$ operation, then $M^{\prime}$ is also an excluded minor for that class (See [1] and [12]). Our research into the above Wheels-and-Whirls Theorem analogue has been approached with a view towards a future splitter theorem extension. To help illuminate this, consider the situation which occurs when one has a 3-connected matroid $M$ with a 3-connected minor $N$ of interest. An element $e$ is then removed from $M$ in such a way that the $N$ minor is retained however a non-trivial 2-separation $(X, Y)$ is exposed in the process. It is readily deduced that, apart from perhaps one single plug element, the elements of the matroid $N$ must lie entirely within $X$, or else entirely within $Y$. Returning then to 
our original matroid $M$, we have an exact 3-separation in which the minor $N$; except at most one plug element, lies entirely on one side. If we then concentrate our efforts on attempting to remove elements from the side of the 3 -separation which does not contain the minor $N$, then we can be sure to retain $N$ in any subsequent removals. In this way, the problem of finding a two-element splitter theorem analogue is reduced to that of a two-element Wheels-and-Whirls Theorem analogue in which we are restricted to only allowing ourselves to remove pairs of elements from one side of a 3-separation. The work we have done herein reveals precisely when such a task is possible. Indeed, from here, we anticipate that a splitter theorem extension is not far off at all, and will for the most part be able to be drawn directly from the structures studied in this investigation. The only obvious extra obstacle to overcome at this point is to understand precisely how a potential plug element interacts with the various possible problematic 3-separating sets.

An interesting related problem to consider would be to study the structures that arise when one disallows the use of the $\Delta-Y$ operation. Much of the simplicity in the statement of Theorem 1.0.1 would no doubt be lost, as more classes of counterexamples are possible; the most obvious two of which being the wheels and the whirls. However, it's conceivable that such a problem could be tackled in time. We should also note that while initial impetus for this research was drawn from other closely related research projects and the current desire to obtain a two-element extension of the splitter theorem, the generalisation of Tutte's Wheels-and-Whirls Theorem contained within this volume is strong, clean, and most certainly of independent interest for matroid theory at large. As such, we expect that it will be of use for researchers in the field for some time to come.

\subsection{Definitions}

We begin by making some definitions which will be used in what follows. Of utmost importance is the notion of matroid connectivity. Let $M$ be a 
matroid on a groundset $E$. The connectivity function; denoted $\lambda_{M}$, which takes subsets of $E$ to the natural numbers, is defined by

$$
\lambda_{M}(X)=r(X)+r(E-X)-r(M) .
$$

A set $X$ or a partition $(X, E-X)$ of $E$ is said to be $k$-separating if $\lambda_{M}(X)<k$ and exactly $k$-separating if $\lambda_{M}(X)=k-1$. A $k$-separating partition $(X, E-$ $X)$ is furthermore a $k$-separation whenever $|X| \geq k \leq|E-X|$, and an exact $k$ separation in the natural sense. The matroid $M$ is $n$-connected provided no $k$ separations of $M$ exist for any $k<n$. We use the term connected in place of $2-$ connected. The matroid $M$ is internally 4-connected if it is 3-connected, and, whenever $(X, E-X)$ is a 3 -separation of $M$, either $|X|=3$ or $|E-X|=3$. A vertical $k$-separation is a triple $(X,\{e\}, Y)$ which partitions $E$ such that both $(X \cup\{e\}, Y)$ and $(X, Y \cup\{e\})$ are exact $k$-separations, $r(X) \geq k \leq r(Y)$, and $e \in \operatorname{cl}(X) \cap \mathrm{cl}(Y)$. A cyclic $k$-separation is a triple $(X,\{e\}, Y)$ which partitions $E$ such that both $(X \cup\{e\}, Y)$ and $(X, Y \cup\{e\})$ are exact $k$-separations, both $X$ and $Y$ contain circuits of $M$, and $e \in \operatorname{cl}^{*}(X) \cap \operatorname{cl}^{*}(Y)$. Note that while elsewhere in the literature a vertical or a cyclic $k$-separation may refer to perhaps something slightly different, in this thesis whenever we use these terms we are explicitly referring to a triple of the aforementioned form. The local connectivity function, denoted $\sqcap_{M}$, which takes pairs of subsets of $E$ to the natural numbers, is defined by

$$
\sqcap_{M}(X, Y)=r(X)+r(Y)-r(X \cup Y) .
$$

Intuitively, it can be helpful to think of the local connectivity function as a measure of the intersection of the closures of the two corresponding sets. Note that when $X$ and $Y$ partition $E$, local connectivity essentially reduces to regular connectivity.

Lemma 1.1.1. Let $M$ be a matroid with $X, Y \subseteq E(M)$. The connectivity function $\lambda_{M}=\lambda$ satisfies the following inequality:

$$
\lambda(X \cap Y)+\lambda(X \cup Y) \leq \lambda(X)+\lambda(Y) .
$$


The above lemma will be used numerous times throughout. In words, it refers to the fact that the connectivity function is submodular. As such, whenever applying Lemma 1.1.1, we shall simply use the phrase 'by submodularity of $\lambda^{\prime}$ or something to that effect. As a direct consequence of submodularity we also have the following useful result which we will apply on a number of occasions, using the term 'by uncrossing' as opposed to directly referencing.

Lemma 1.1.2. Let $M$ be a 3-connected matroid, and let $X$ and $Y$ be 3separating subsets of $E(M)$. Then the following properties hold:

(i) if $|X \cap Y| \geq 2$, then $X \cup Y$ is 3-separating, and

(ii) if $|E(M)-(X \cup Y)| \geq 2$, then $X \cap Y$ is 3-separating.

The following result describing the relationship between vertical and cyclic 3-separations is straightforward, but should be noted as it is frequently used implicitly and without reference.

Lemma 1.1.3. Let $(X,\{e\}, Y)$ be a partition of a matroid $M$. Then $(X,\{e\}, Y)$ is a vertical 3-separation of $M$ if and only if it is a cyclic 3separation of $M^{*}$.

Let $M$ be matroid with an element $e$ such that there exists an exact 3separation $(X, Y)$ of $M \backslash e$. We say that the element $e$ blocks the 3-separation $(X, Y)$, or blocks the exactly 3 -separating set $X$ if neither $X$ nor $Y$ is exactly 3 -separating in $M$. The following equivalent definition of a blocking element is readily checked.

Lemma 1.1.4. A 3-separation $(X, Y)$ of a matroid $M \backslash e$ is blocked by e in $M$ if and only if $e \notin(\operatorname{cl}(X) \cup \operatorname{cl}(Y))$.

A subset $X$ of a matroid $M$ is fully closed if $\operatorname{cl}(X)=X=\operatorname{cl}^{*}(X)$. The full closure of a set $X$; denoted $\operatorname{fcl}(X)$, is the intersection of all fully closed sets 
which contain $X$. We shall write $x \in \mathrm{cl}^{(*)}(X)$ to denote that $x \in \operatorname{cl}(X)$ or $x \in \mathrm{cl}^{*}(X)$. A 3-separating subset $X$ of a matroid $M$ is said to be sequential if there exists an ordering $\left(x_{1}, \ldots, x_{n}\right)$ of the elements of $X$ such that $\left\{x_{1}, \ldots, x_{i}\right\}$ is 3 -separating for all $i$ where $1 \leq i \leq n$. In such a situation, $\left(x_{1}, \ldots, x_{n}\right)$ is a sequential ordering of $X$. A matroid $M$ is itself said to be sequential if $E(M)$ has some sequential ordering. The following result on sequential orderings is straightforward.

Lemma 1.1.5. Let $\left(x_{1}, \ldots, x_{n}\right)$ be a sequential ordering of a sequential set $X$ in a 3-connected matroid $M$. Then, for $i \in\{3, \ldots, n-2\}$, one of the following holds:

(i) $i \in \operatorname{cl}\left(\left\{x_{1}, \ldots, x_{i-1}\right\}\right) \cap \operatorname{cl}\left(E(M)-\left\{x_{1}, \ldots, x_{i}\right\}\right)$, or

(ii) $i \in \mathrm{cl}^{*}\left(\left\{x_{1}, \ldots, x_{i-1}\right\}\right) \cap \mathrm{cl}^{*}\left(E(M)-\left\{x_{1}, \ldots, x_{i}\right\}\right)$.

If outcome (i) of Lemma 1.1.5 holds for a particular element $x_{i}$ of a sequential set $X$, then $x_{i}$ is said to be a guts element. In the case that (ii) holds, $x_{i}$ is a coguts element.

Let $M$ be a matroid with a subset $Z \subseteq E(M)$ where $|Z| \geq 3$. The set $Z$ is a segment if every triple of $Z$ is a triangle. Dually, $Z$ is a cosegment if every triple of $Z$ is a triad. A subset $D$ of $M$ is a quad if $|D|=4$ and $D$ is both a circuit and a cocircuit.

Definition 1.1.6. Let $F=\left(f_{1}, \ldots, f_{n}\right)$ be an ordered sequence of distinct elements of $M$ where $n \geq 3$. Then $F$ is a fan if for all $i \in\{1, \ldots, n-2\}$,

(i) $\left\{f_{i}, f_{i+1}, f_{i+2}\right\}$ is a triangle or a triad, and

(ii) whenever $\left\{f_{i}, f_{i+1}, f_{i+2}\right\}$ is a triangle, $\left\{f_{i+1}, f_{i+2}, f_{i+3}\right\}$ is a triad, and, whenever $\left\{f_{i}, f_{i+1}, f_{i+2}\right\}$ is a triad, $\left\{f_{i+1}, f_{i+2}, f_{i+3}\right\}$ is a triangle.

The fan $F=\left(f_{1}, \ldots, f_{n}\right)$ is maximal in $M$ provided there does not exist a fan $G=\left(g_{1}, \ldots, g_{m}\right)$ such that $\left\{g_{1}, \ldots, g_{m}\right\}$ properly contains $\left\{f+1, \ldots, f_{n}\right\}$. If $F=\left(f_{1}, \ldots, f_{n}\right)$ is a maximal fan, then $f_{1}$ and $f_{n}$ are said to be the ends of the fan. For a proof of the following result, see [14]. 
Lemma 1.1.7. Let $M$ be a 3-connected matroid which is not a wheel or a whirl, and let $F$ be a maximal fan in $M$ containing at least four elements. If $e$ is an end of $F$ and $e$ is contained in a triangle of $M$, then $M \backslash e$ is 3-connected.

Definition 1.1.8. Let $F=\left(f_{1}, f_{2}, \ldots, f_{n}\right)$ be an ordered sequence of distinct elements of a 3-connected matroid $M$ where $n \geq 4$. Then $F$ is a flan of $M$ if the following hold.

(i) If $i$ is an odd member of $\{1, \ldots, n-2\}$, then $\left\{f_{i}, f_{i+1}, f_{i+2}\right\}$ is a triad.

(ii) If $i$ is an even member of $\{4, \ldots, n\}$, then $f_{i} \in \operatorname{cl}\left(\left\{f_{1}, f_{2}, \ldots, f_{i-1}\right\}\right)$.

The flan $F$ is maximal if there is no element $f_{n+1}$ such that $\left(f_{1}, f_{2}, \ldots, f_{n}, f_{n+1}\right)$ is a flan. $\mathrm{A}$ fan whose ordering begins with a triad is of course a specific example of a flan. Flans in general are an important structure encountered throughout this work and we shall take a closer look at some results related to them in Section 2.4.

Definition 1.1.9. A paddle in a 3-connected matroid $M$ is a partition $\left(P_{1}, \ldots, P_{n}\right)$ of $E(M)$ where $n \geq 3$ such that the following hold:

(i) $\left|P_{i}\right| \geq 2$ for every $i \in\{1, \ldots, n\}$,

(ii) $\lambda\left(\bigcup_{s \in S} P_{s}\right) \leq 2$ for every subset $S$ of $\{1, \ldots, n\}$, and

(iii) $\sqcap\left(P_{i}, P_{j}\right)=2$ for all distinct $i, j \in\{1, \ldots, n\}$.

Let $\Delta=\{a, b, c\}$ be a triangle of $M$ and consider a copy of $M\left(K_{4}\right)$ having $\Delta$ as a triangle with $\left\{a^{\prime}, b^{\prime}, c^{\prime}\right\}$ as the complementary triad labelled such that $\left\{a, b^{\prime}, c^{\prime}\right\},\left\{a^{\prime}, b, c^{\prime}\right\}$ and $\left\{a^{\prime}, b^{\prime}, c\right\}$ are triangles. Let $P_{\Delta}\left(M, M\left(K_{4}\right)\right)$ denote the generalised parallel connection of $M$ with $M\left(K_{4}\right)$ across the triangle $\Delta$. Consider then the matroid $P_{\Delta}\left(M, M\left(K_{4}\right)\right) \backslash \Delta$ where the elements $a^{\prime}, b^{\prime}$ and $c^{\prime}$ are relabelled as $a, b$ and $c$ respectively. This resulting matroid, denoted $\Delta M$, is said to be obtained from $M$ via performing a $\Delta-Y$ exchange on 
the triangle $\Delta$. Dually, a matroid $N$ is obtained from $M$ via performing a $Y-\Delta$ exchange on the triad $\{a, b, c\}$ if $N^{*}$ is obtained from $M^{*}$ via a $\Delta-Y$ exchange on $\{a, b, c\}$.

Definition 1.1.10. Let $M$ be a 3-connected matroid with $x, y \in E(M)$. The pair $\{x, y\}$ is a detachable pair if either $M \backslash x, y$ or $M / x, y$ is 3-connected.

As alluded to in the introduction, the bulk of the work contained in this thesis is concerned with locating detachable pairs within a specific side of a 3-separation and studying the structures that arise when such a task is not possible. Quads are the simplest examples of exactly 3-separating sets which yield no detachable pair. As shall become clear in the later chapters, the other troublesome counterexamples can be classified into four specific subtypes which we capture in the following four definitions.

Definition 1.1.11. Let $M$ be a matroid with a 3 -separating subset $X$. The set $X$ is a spike-like 3 -separator if there exists a partition $\left\{X_{1}, \ldots, X_{n}\right\}$ of $X$ with $n \geq 3$ such that the following properties hold:

(i) $\left|X_{i}\right|=2$ for every $i \in\{1, \ldots, n\}$, and

(ii) $X_{i} \cup X_{j}$ is a quad for all distinct $i, j \in\{1, \ldots, n\}$.

In such a situation, $\left\{X_{1}, \ldots, X_{n}\right\}$ is said to be the associated partition of the spike-like 3-separator $X$. Furthermore, if $E(M)$ is a spike-like 3-separator, then $M$ itself is a spike.

Definition 1.1.12. Let $M$ be a 3 -connected matroid containing a quad $P$ with $q_{1}, q_{2} \in E(M)-P$. Then $X=P \cup\left\{q_{1}, q_{2}\right\}$ is a partial spider-like 3-separator with associated partition $\left\{P,\left\{q_{1}, q_{2}\right\}\right\}$ if there exists a labelling $P=\left\{p_{1}, p_{2}, p_{3}, p_{4}\right\}$ of $P$ such that:

(i) $\left\{p_{1}, p_{2}, q_{1}, q_{2}\right\}$ and $\left\{p_{3}, p_{4}, q_{1}, q_{2}\right\}$ are circuits, and

(ii) $\left\{p_{1}, p_{3}, q_{1}, q_{2}\right\}$ and $\left\{p_{2}, p_{4}, q_{1}, q_{2}\right\}$ are cocircuits. 


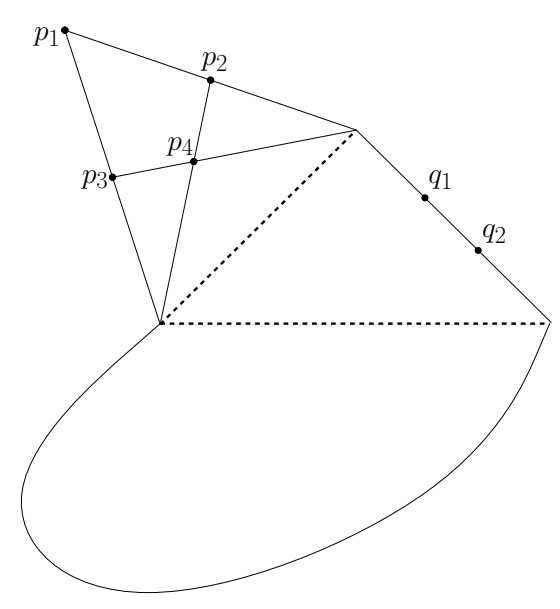

(a) Partial Spider-Like 3-Separator

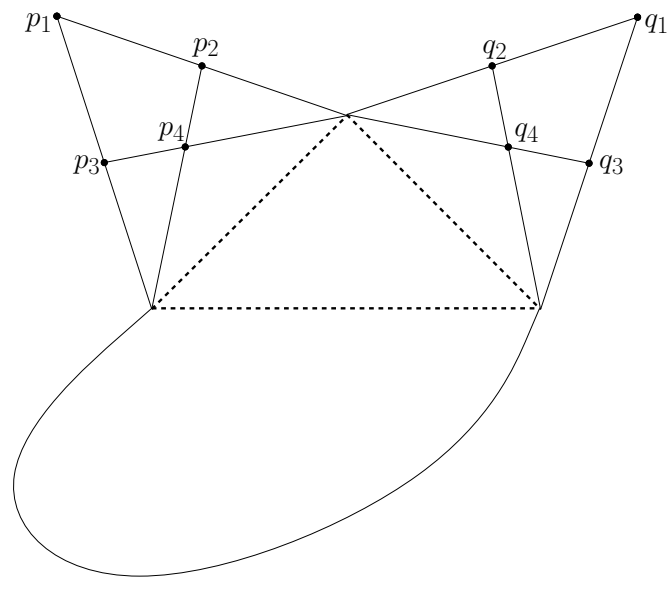

(b) Spider-Like 3-Separator

Figure 1.1: Geometric Representations of Definitions 1.1.12 and 1.1.13

Definition 1.1.13. Let $M$ be a 3-connected matroid containing disjoint quads $P$ and $Q$. Then $X=P \cup Q$ is a spider-like 3 -separator with associated partition $\{P, Q\}$ if there exists a labelling $P=\left\{p_{1}, p_{2}, p_{3}, p_{4}\right\}$ and $Q=\left\{q_{1}, q_{2}, q_{3}, q_{4}\right\}$ of $P$ and $Q$ such that:

(i) $\left\{p_{1}, p_{2}, q_{1}, q_{2}\right\},\left\{p_{1}, p_{2}, q_{3}, q_{4}\right\},\left\{p_{3}, p_{4}, q_{1}, q_{2}\right\}$ and $\left\{p_{3}, p_{4}, q_{3}, q_{4}\right\}$ are circuits, and

(ii) $\left\{p_{1}, p_{3}, q_{1}, q_{3}\right\},\left\{p_{1}, p_{3}, q_{2}, q_{4}\right\},\left\{p_{2}, p_{4}, q_{1}, q_{3}\right\}$ and $\left\{p_{2}, p_{4}, q_{2}, q_{4}\right\}$ are cocircuits.

Definition 1.1.14. Let $(X, Y)$ be an exact 3-separation of the matroid $M$. Then $X$ is a twisted 3-separator if $|X|=6$ and there exists a labelling $\left\{r_{1}, r_{2}, s_{1}, s_{2}, t_{1}, t_{2}\right\}$ of the elements of $X$ such that the following hold.

(i) $\left\{r_{1}, r_{2}, s_{2}, t_{1}\right\},\left\{s_{1}, s_{2}, r_{1}, t_{2}\right\}$ and $\left\{t_{1}, t_{2}, s_{1}, r_{2}\right\}$, are the circuits of $M$ contained in $X$.

(ii) $\left\{r_{1}, r_{2}, s_{1}, s_{2}\right\},\left\{s_{1}, s_{2}, t_{1}, t_{2}\right\}$ and $\left\{t_{1}, t_{2}, r_{1}, r_{2}\right\}$ are the cocircuits of $M$ contained in $X$. 


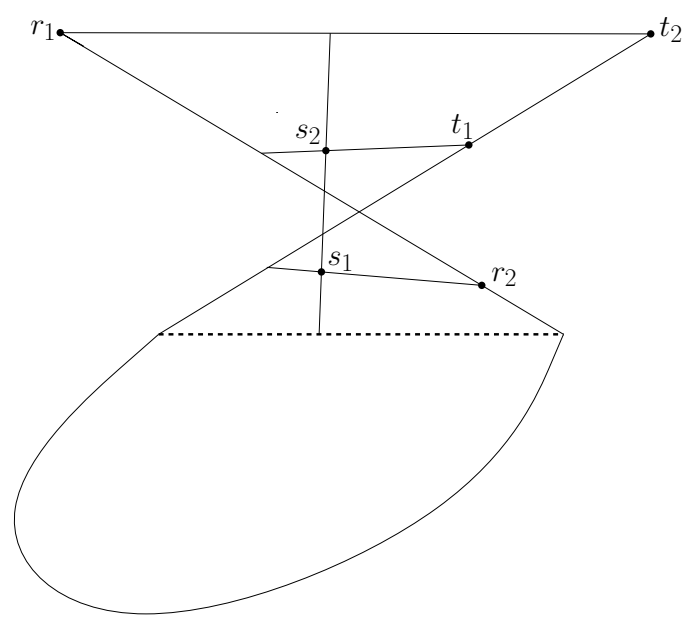

Figure 1.2: Geometric Representation of Definition 1.1.14

It will become much more clear in Chapter 2 precisely how a twisted 3separator is configured and how it can be constructed as an extension of a flan. With that we conclude our definitions. Anything not explicitly defined above accords to the conventions and notation as described in [11].

\subsection{Further Preliminary Lemmas}

We catalogue some important lemmas which will be needed in the following chapters. First and foremost amongst these is the following fundamental result which is used extensively throughout.

Lemma 1.2.1. Let $M$ be a matroid. If $C$ is a circuit of $M$ and $C^{*}$ a cocircuit of $M$, then $\left|C \cap C^{*}\right| \neq 1$.

We shall use the phrase by orthogonality whenever applying Lemma 1.2.1. The next three results are straightforward and well known. We note them here as they are used numerous times; all the while implicitly and without reference.

Lemma 1.2.2. Let $e$ be an element of a matroid $M$, and let $X$ and $Y$ be 
disjoint sets whose union is $E(M)-\{e\}$. Then $e \in \operatorname{cl}(X)$ if and only if $e \notin \mathrm{cl}^{*}(Y)$.

Lemma 1.2.3. Let $X$ be an exactly 3-separating set in a 3-connected matroid $M$, and suppose that $e \in E(M)-X$. Then $X \cup\{e\}$ is 3-separating if and only if $e \in \mathrm{cl}^{(*)}(X)$.

Lemma 1.2.4. Let $(X, Y)$ be an exactly 3-separating partition of a 3connected matroid $M$. Suppose $|X| \geq 3$ and $x \in X$. Then:

(i) $x \in \mathrm{cl}^{(*)}(X-\{x\})$; and

(ii) $(X-\{x\}, Y \cup\{x\})$ is exactly 3-separating if and only if $x$ is in exactly one of $\operatorname{cl}(X-\{x\}) \cap \operatorname{cl}(Y)$ and $\operatorname{cl}^{*}(X-\{x\}) \cap \operatorname{cl}^{*}(Y)$.

All of the results mentioned hereafter will be explicitly referenced at the appropriate time. The next seven lemmas are elementary.

Lemma 1.2.5. Let $(P, Q)$ be a k-separating partition of a matroid $M$. Then for any subset $X \subseteq E(M),(P \cap X, Q \cap X)$ is a k-separating partition of the restricted matroid $M \mid X$.

Lemma 1.2.6. Let $(X, Y)$ be a 2-separation in a connected matroid $M$ where $M$ contains no series or parallel pairs. Then $(\mathrm{fcl}(X), Y-\mathrm{fcl}(X))$ is also a 2-separation of $M$.

Lemma 1.2.7. Let $M$ be a matroid with $x \in E(M)$. If $Y \subseteq E(M \backslash x)$ is fully closed in $M \backslash x$ and $x \in \operatorname{cl}(Y)$, then $Y \cup\{x\}$ is fully closed in $M$.

Lemma 1.2.8. Let $X$ be a fully closed subset of a matroid $M$ and $x \in X$. Then $X-\{x\}$ is fully closed in both $M \backslash x$ and $M / x$.

Lemma 1.2.9. Let $(S, T, U)$ be a paddle in a 3-connected matroid $M$. Then $\mathrm{cl}^{*}(X)=X$ for each $X \in\{S, T, U\}$.

Lemma 1.2.10. Let $(X, Y)$ be an exact 3-separation of a 3-connected matroid $M$. Then $\left(\operatorname{cl}(X) \cap \mathrm{cl}^{*}(X)\right)-X=\emptyset$. 
Lemma 1.2.11. Let e be an element of a 3-connected matroid $M$. If $\operatorname{si}(M / e)$ is not 3-connected, then there exists a vertical 3-separation $(X,\{e\}, Y)$ of $M$. Similarly, if $\operatorname{co}(M \backslash e)$ is not 3-connected, there exists a cyclic 3-separation $(P,\{e\}, Q)$ of $M$.

The following two results relating to connectivity under $\Delta-Y$ operations are straightforward and readily deduced. The interested reader should see for example Chapter 4, Section 3 of [8].

Lemma 1.2.12. Let $T$ be a triangle of a matroid $M$ with some $t \in T$. Let $M^{\prime}$ denote the matroid obtained by performing a $\Delta-Y$ exchange on the triangle $T$. Then $M \backslash t \cong M^{\prime} / t$. In particular, if $T=\left\{t, t_{1}, t_{2}\right\}$, then $M \backslash t$ is isomorphic to $M^{\prime} / t$ under the map which interchanges $t_{1}$ and $t_{2}$ and leaves every other element fixed.

Lemma 1.2.13. Let $M$ be a 3-connected matroid containing a fan $F=$ $\left(f_{1}, \ldots, f_{k}\right)$ where $k \geq 4$ and $\left\{f_{1}, f_{2}, f_{3}\right\}$ is a triangle. Let $M^{\prime}$ denote the matroid obtained by performing a $Y-\Delta$ exchange on the triad $\left\{f_{2}, f_{3}, f_{4}\right\}$. Then:

(i) if $k=4$ and $F$ is maximal, the matroid $M^{\prime}$ is 3-connected up to the parallel pair $\left\{f_{1}, f_{4}\right\}$,

(ii) if $k>5$, the matroid $M^{\prime}$ is 3-connected up to the two distinct parallel pairs $\left\{f_{1}, f_{4}\right\}$ and $\left\{f_{2}, f_{5}\right\}$, and

(iii) if $k=5$ and $F$ is maximal, provided there does not exist a triangle in $M$ which contains $\left\{f_{2}, f_{4}\right\}$, the matroid $M^{\prime}$ is 3-connected up to the two distinct parallel pairs $\left\{f_{1}, f_{4}\right\}$ and $\left\{f_{2}, f_{5}\right\}$. In the case that such a triangle $\left\{f_{2}, f_{4}, z\right\}$ does exist for some $z$, the matroid $M^{\prime}$ is 3-connected up to the three distinct parallel pairs $\left\{f_{1}, f_{4}\right\},\left\{f_{2}, f_{5}\right\}$ and $\left\{f_{3}, z\right\}$.

A proof of the following can be found in [23]. 
Lemma 1.2.14. Let $M$ be a 3-connected matroid with $r(M) \geq 4$. Suppose that $C^{*}$ is a rank-3 cocircuit of $M$. If there exists some $c \in C^{*}$ such that $r\left(C^{*}-\{c\}\right)=3$, then $\operatorname{co}(M \backslash c)$ is 3-connected.

The next result is known as Bixby's Lemma [3], and will be referred to as such whenever used.

Lemma 1.2.15. Let $e$ be an element of a 3-connected matroid $M$. Then either $\operatorname{si}(M / e)$ or $\operatorname{co}(M \backslash e)$ is 3-connected.

The following two well-known results are due to Tutte [19], and will again be referenced via their standardised names. The first is Tutte's Triangle Lemma.

Lemma 1.2.16. Let $\{a, b, c\}$ be a triangle in a 3-connected matroid $M$ where $|E(M)| \geq 4$. If neither $M \backslash a$ nor $M \backslash b$ is 3-connected, then $M$ has a triad which contains $a$ and exactly one element from $\{b, c\}$.

It's worth noting that, in practice, the way we will often apply Lemma 1.2.16 is when examining a triangle which we know cannot be contained in a 4-element fan. In such a situation, Tutte's Triangle Lemma then guarantees us that we can always delete at least two elements from that triangle without exposing a 2-separation. The second of Tutte's results is known as Tutte's Wheels-and-Whirls Theorem. It is of course precisely the result for which we are seeking a two-element analogue in this thesis.

Lemma 1.2.17. Let $M$ be a 3-connected matroid. Provided $M$ is not a wheel or a whirl, there exists some element $e \in E(M)$ such that either $M \backslash e$ or $M / e$ is 3-connected.

We round out this introductory chapter with seven results of varying scope that will be required in what follows.

Lemma 1.2.18. Let $M$ be a 3-connected matroid with $A=\{a, b, c, d, e\} \subseteq$ $E(M)$ and $Z=E(M)-A$. Then $r(Z)=r(M)-3$ if and only if $A$ is a coline. 
Proof. The set $\{a, b, c\}$ is not a triad if and only if it does not contain a cocircuit; if and only if $Z \cup\{d, e\}$ is spanning; if and only if $r(Z) \geq r(M)-2$. As this applies to every triple of $A$, it follows that $A$ is itself a coline if and only if $r(Z)=r(M)-3$.

Lemma 1.2.19. Let $M$ be a 3-connected matroid with a pair of disjoint triads $S=\left\{s_{1}, s_{2}, s_{3}\right\}$ and $T=\left\{t_{1}, t_{2}, t_{3}\right\}$. If

(i) $\left\{s_{1}, s_{2}, t_{1}, t_{2}\right\}$ is a circuit of $M$, and

(ii) $s_{3}$ is not in a triangle of $M$,

then $M / s_{3}$ is 3-connected.

Proof. Note that $\sqcap(S, T) \geq 1$. Suppose that $(X, Y)$ is a 2-separation in $M / s_{3}$ with $|X \cap T| \geq 2 . M / s_{3}$ contains no series pairs, and, because $s_{3}$ is not contained in any triangles, no parallel pairs either. Now by an application of Lemma 1.2.6 and a potential relabelling, we may assume that $X$ is fully closed and so $T \subseteq X$. If $\sqcap(S, T)=2$, then $\left\{s_{1}, s_{2}\right\} \subseteq \operatorname{cl}_{M / s_{3}}(T) \subseteq X$, implying that $\left(X \cup\left\{s_{3}\right\}, Y\right)$ is a 2-separation of $M$. Otherwise $\sqcap(S, T)=1$. To avoid the same contradiction, the pair $\left\{s_{1}, s_{2}\right\}$ must cross the 2-separation $(X, Y)$, so without loss of generality, $s_{1} \in X, s_{2} \in Y$. But $X$ is fully closed in $M / s_{3}$, contradicting the fact that $\left\{s_{1}, s_{2}, t_{1}, t_{2}\right\}$ is a circuit.

Lemma 1.2.20. Let $M$ be a 3-connected matroid with a pair $\left\{p_{1}, p_{2}\right\}$ of elements such that there are distinct cocircuits $\left\{a_{1}, a_{2}, p_{1}, p_{2}\right\}$ and $\left\{b_{1}, b_{2}, p_{1}, p_{2}\right\}$ and $M \backslash p_{1}, p_{2}$ is 3-connected up to the series pairs $\left\{a_{1}, a_{2}\right\}$ and $\left\{b_{1}, b_{2}\right\}$. Then either

(i) there exists $x \in\left\{a_{1}, a_{2}, b_{1}, b_{2}\right\}$ such that $M / x$ is 3-connected, or

(ii) up to labelling, $\left\{a_{1}, b_{1}, p_{1}\right\}$ and $\left\{a_{2}, b_{2}, p_{2}\right\}$ are triangles of $M$.

Proof. We shall assume that no element of $\left\{a_{1}, a_{2}, b_{1}, b_{2}\right\}$ can be contracted from $M$ without losing 3-connectivity. Suppose that $a_{1}$ is not 
in a triangle and consider $M / a_{1}$. Because $\left\{b_{1}, b_{2}, p_{1}, p_{2}\right\}$ is a cocircuit of $M$, it is also a cocircuit of $M / a_{1}$ and so neither of $p_{1}$ or $p_{2}$ is contained in $\operatorname{cl}_{M / a_{1}}\left(E\left(M / a_{1}\right)-\left\{b_{1}, b_{2}, p_{1}, p_{2}\right\}\right)$. As the only 2-separation of $M / a_{1} \backslash p_{1}, p_{2}$ is $\left(\left\{b_{1}, b_{2}\right\}, E\left(M / a_{1} \backslash p_{1}, p_{2}\right)-\left\{b_{1}, b_{2}\right\}\right)$, it now follows that $\left\{p_{1}, p_{2}\right\} \subseteq \operatorname{cl}_{M / a_{1}}\left(\left\{b_{1}, b_{2}\right\}\right)$, implying in turn that $\left\{p_{1}, p_{2}\right\} \subseteq \operatorname{cl}_{M}\left(\left\{b_{1}, b_{2}, a_{1}\right\}\right)$, and that $r_{M}\left(\left\{b_{1}, b_{2}, p_{1}, p_{2}\right\}\right)=3$ with $a_{1} \in \operatorname{cl}\left(\left\{b_{1}, b_{2}, p_{1}, p_{2}\right\}\right)$. Suppose now that $a_{2}$ is in a triangle. Then this triangle must contain one of $\left\{p_{1}, p_{2}\right\}$ as $\left\{a_{1}, a_{2}, p_{1}, p_{2}\right\}$ is a cocircuit, and again by orthogonality, it must also contain one of $\left\{b_{1}, b_{2}\right\}$. Now $a_{2} \in \operatorname{cl}\left(\left\{a_{1}, b_{1}, b_{2}, p_{1}, p_{2}\right\}\right)$ which has rank three; giving a contradiction as $r\left(\left\{a_{1}, a_{2}, b_{1}, b_{2}\right\}\right)=4$. We deduce that $a_{2}$ cannot be in a triangle of $M$. But now we may repeat the above argument with $a_{2}$ in the place of $a_{1}$, leading to the deduction that $a_{2} \in \operatorname{cl}\left(\left\{b_{1}, b_{2}, p_{1}, p_{2}\right\}\right)$, again leading to the contradiction that $r\left(\left\{a_{1}, a_{2}, b_{1}, b_{2}\right\}\right)=3$. Thus $a_{1}$ and indeed $a_{2}$ must be in triangles and by orthogonality and up to a labelling it is readily checked that these triangles must be $\left\{a_{1}, b_{1}, p_{1}\right\}$ and $\left\{a_{2}, b_{2}, p_{2}\right\}$.

Lemma 1.2.21. Let $M$ be a 3-connected matroid with a 3-separating set $\left\{a_{1}, a_{2}, a_{3}, a_{4}\right\}$ such that $\left\{a_{1}, a_{2}, a_{3}\right\}$ is a triad and $a_{4} \in \operatorname{cl}\left(\left\{a_{1}, a_{2}, a_{3}\right\}\right)$. If $i \in\{1,2,3\}$, and $a_{i}$ is not in a triangle, then $M / a_{i}$ is 3-connected.

Proof. Suppose that $a_{1}$ is not in a triangle and $M / a_{1}$ is not 3 -connected. Then neither is $\operatorname{si}\left(M / a_{1}\right)$, and we have a vertical 3-separation $\left(X,\left\{a_{1}\right\}, Y\right)$ of $M$. Both $X$ and $Y$ must contain one element of the triad $\left\{a_{1}, a_{2}, a_{3}\right\}$. Say $a_{2} \in X, a_{3} \in Y$. Observe now that the presence of $a_{4} \in X$ implies that $a_{3} \in \operatorname{cl}\left(X \cup\left\{a_{1}\right\}\right) \cap \operatorname{cl}^{*}\left(X \cup\left\{a_{1}\right\}\right)$, which contradicts Lemma 1.2.10. Similarly, $a_{4} \in Y$ gives $a_{2} \in \operatorname{cl}\left(Y \cup\left\{a_{1}\right\}\right) \cap \operatorname{cl}^{*}\left(Y \cup\left\{a_{1}\right\}\right)$.

Lemma 1.2.22. Let $M$ be a 3-connected matroid. If $(X, Y)$ is a 3-separation of $M$ where $\mathrm{cl}(X)$ contains no triangles and $|X| \geq 4$, then there exists a 3-separating set $X^{\prime} \subseteq X$ with $\left|X^{\prime}\right| \geq 3$ having the property that $M / x$ is 3-connected for all $x \in X^{\prime}$.

Proof. Assume that the lemma fails. From the finite collection of counterexamples, let $(X, Y)$ be chosen such that $|X|$ is minimal. There must exist some 
$x \in X-\operatorname{cl}(Y)$ such that $M / x$ is not 3-connected, and as $\operatorname{cl}(X)$ contains no triangles and $x \notin \mathrm{cl}(Y)$ it follows that $x$ cannot be in any triangle of $M$. Therefore there exists some vertical 3-separation $(P,\{x\}, Q)$ of $M$. Clearly we cannot have $P \subseteq Y$ or $Q \subseteq Y$ as $x \notin \operatorname{cl}(Y)$. So $P \cap X \neq \emptyset \neq Q \cap X$. Suppose also that $P \cap Y \neq \emptyset \neq Q \cap Y$, so that the vertical 3-separation $(P,\{x\}, Q)$ crosses the 3 -separation $(X, Y)$. If $|X \cap P|=1$, then it must be the case that $|X \cap Q| \geq 2 \leq|Y \cap P|$. Similarly, if $|Y \cap Q|=1$, then we must have $|X \cap Q| \geq 2 \leq|Y \cap P|$. Thus, in any case, we may assume up to an elementary relabelling that $|X \cap P| \geq 2 \leq|Y \cap Q|$. It follows then, by uncrossing these separations, that $X \cap P$ is exactly 3 -separating with $x \in \operatorname{cl}(X \cap P)$. As $X$ contains no triangles, it is now clear that $(X \cap P,\{x\}, Y \cup Q)$ is a vertical 3 -separation of $M$. Therefore we may assume that whatever the case may be, there must always exist some vertical 3-separation $(U,\{x\}, V)$ of $M$ with $U \subseteq X$. Obviously $U \cup\{x\} \neq X$; as otherwise $x \in \operatorname{cl}(Y)$. Therefore $U \cup\{x\}$ is a proper 3 -separating subset of $X$ containing at least four elements; a situation which contradicts our original choice of $X$.

Lemma 1.2.23. Let $(X, Y)$ be a 3-separation of a 3-connected matroid $M$. If $\mathrm{fcl}(X)$ contains no triads or triangles, then there exists a 3-separating set $X^{\prime} \subseteq X$ with $\left|X^{\prime}\right| \geq 4$ having the property that for all $x \in X^{\prime}$, both $M \backslash x$ and $M / x$ are 3 -connected.

Proof. As $X$ contains no triads or triangles we have $|X| \geq 4$. By Lemma 1.2.22 then, $X$ contains a 3-separating subset $X^{\prime} \subseteq X$ with $\left|X^{\prime}\right| \geq 3$ having the property that $M / x$ is 3 -connected for all $x \in X^{\prime}$. In fact it must be the case that $\left|X^{\prime}\right| \geq 4$ as $X$ does not have any 3-element 3-separating subsets. Applying the dual of Lemma 1.2.22 now to the set $X^{\prime}$, we find a 3-separating subset $X^{\prime \prime} \subseteq X^{\prime} \subseteq X$ with the property that for all $x \in X^{\prime \prime}$, both $M / x$ and $M \backslash x$ are 3 -connected as required.

Lemma 1.2.24. Let $\Gamma$ be a collection of cocircuits of a matroid $M$ such that for all $C \in \Gamma$, the set $C-\bigcup_{K \in \Gamma-\{C\}} K$ is non-empty. Then $E(M)-\bigcup_{K \in \Gamma} K$ is a flat of rank at most $r(M)-|\Gamma|$. 
When the Lemma 1.2.24 is used on a collection $\Gamma$ of cocircuits of a matroid $M$, for the sake of readability, we shall simply say ' $E(M)-\bigcup_{K \in \Gamma} K$ is a flat of rank at most $r(M)-|\Gamma|$ ', without explicit reference to the lemma. When doing so, it is implicit that the collection $\Gamma$ satisfies the conditions of 1.2.24. The lemma itself follows straightforwardly from the lattice of flats structure of a matroid. See [11] for details. 


\section{Chapter 2}

\section{Obstacles}

In this chapter we shall study some very precise structures that cause varying degrees of difficulty when searching for detachable pairs.

\subsection{Triangles}

The first of these structures is the triangle (and dually, the triad). As one might expect, certain arrangements of triangles and triads in a matroid can be troublesome. Wheels and whirls are obviously an issue for a start. We overcome this triangle (and triad) problem entirely by allowing ourselves use of the $\Delta-Y$ operation.

Theorem 2.1.1. Let $M$ be a 3-connected matroid with $|E(M)|>9$. If $M$ contains a triangle, then either

(i) $M$ contains a detachable pair, or

(ii) there exists a matroid $M^{\prime}$ obtained via a single $\Delta-Y$ exchange on $M$ or $M^{*}$ such that $M^{\prime}$ contains a detachable pair.

Proof. Assume that the theorem fails. Suppose to begin with that $M$ contains a fan $F$ with at least 6 elements. Up to duality, we may assume that 
$F$ is such that its first three elements $f_{1}, f_{2}, f_{3}$ form a triangle. Now let $M^{\prime}$ be the matroid obtained via performing a $Y-\Delta$ on the $\operatorname{triad}\left\{f_{2}, f_{3}, f_{4}\right\}$. By Lemma 1.2.13, $M^{\prime}$ is 3-connected up to the two distinct non-trivial parallel classes $\left\{f_{1}, f_{4}\right\}$ and $\left\{f_{2}, f_{5}\right\}$ and thus $M^{\prime}$ has a detachable pair. Next, suppose that $M$ contains a maximal 4-element fan $F=\left(f_{1}, f_{2}, f_{3}, f_{4}\right)$. Again we may assume that $F$ begins with a triangle $\left\{f_{1}, f_{2}, f_{3}\right\}$. By Lemma 1.2.21, $M / f_{4}$ must be 3 -connected. Thus if we let $M^{\prime}$ be the matroid obtained through performing a $Y-\Delta$ exchange on the triad $\left\{f_{2}, f_{3}, f_{4}\right\}$, we see by Lemma 1.2.12 that $M^{\prime} \backslash f_{4}$ is 3-connected. As $F$ is maximal, it now follows from Tutte's Triangle Lemma that either $M^{\prime} \backslash f_{1}, f_{4}$ or $M^{\prime} \backslash f_{2}, f_{4}$ must be 3connected. This gives us a detachable pair in $M^{\prime}$. Thus, in the event that $M$ contains a fan $F$ of at least four elements, the only remaining possibility is that $F=\left(f_{1}, \ldots, f_{5}\right)$ and $F$ is maximal. We may again assume that $\left\{f_{1}, f_{2}, f_{3}\right\}$ is a triangle. It now follows from Lemma 1.2 .13 that by letting $M^{\prime}$ denote the matroid obtained via performing a $Y-\Delta$ on the triad $\left\{f_{2}, f_{3}, f_{4}\right\}$, the matroid $M^{\prime} \backslash f_{2}, f_{4}$ is 3-connected unless there exists a triangle $\left\{f_{2}, f_{4}, z\right\}$ in $M$ for some $z \in E(M)-F$. Since we are under the assumption that $M^{\prime}$ does not contain a detachable pair, we now study the situation where the triangle $\left\{f_{2}, f_{4}, z\right\}$ is indeed present.

2.1.1.1. There exists $f_{0}, f_{6} \in E(M)-F$ such that $\left(f_{0}, f_{1}, \ldots, f_{5}, f_{6}\right)$ is a maximal fan of $M \backslash z$.

Proof. The matroid $M \backslash z$ is certainly 3-connected. If $F$ is a maximal fan of $M \backslash z$, it follows from Lemma 1.1.7 that $M$ has a detachable pair. So $F$ is not maximal. Suppose that $M \backslash z$ is a wheel or a whirl. Then, as $|E(M)|>9$, there must exist some fan $H$ of $M$ where $H \cap\left\{f_{2}, f_{3}, f_{4}, z\right\}=\emptyset$ and $|H| \geq 6$. But this contradicts our current assumption that the largest fan in $M$ has 5 elements. So $M \backslash z$ is not a wheel or a whirl. The fan $F$ is contained in some maximal fan $G$. By Lemma 1.1.7, $|G| \geq 7$. If there exists some triad $\Gamma$ of $M \backslash z$ such that $\Gamma$ is a triad of consecutive elements in the fan $G$ where $\Gamma \cap F=\emptyset$, then by orthogonality, the element $z$ cannot block $\Gamma$ and so $\Gamma$ is 
also a triad of $M$. Thus, as the fan $G=\left(g_{1}, \ldots, g_{m}\right)$ must, by Lemma 1.1.7, be such that both $\left\{g_{1}, g_{2}, g_{3}\right\}$ and $\left\{g_{m-2}, g_{m-1}, g_{m}\right\}$ are triads of $M \backslash z$, it follows that if $|G| \geq 8$, then at least one of $\left\{g_{1}, g_{2}, g_{3}\right\}$ and $\left\{g_{m-2}, g_{m-1}, g_{m}\right\}$ is a triad of $M$ which is disjoint from $F$. Assume without loss of generality that $\left\{g_{1}, g_{2}, g_{3}\right\}$ is a triad of $M$. As the fan $G$ is maximal in $M \backslash z$, it now follows that $\operatorname{cl}_{M \backslash z}\left(\left\{g_{1}, g_{2}, g_{3}\right\}\right)=\operatorname{cl}_{M}\left(\left\{g_{1}, g_{2}, g_{3}\right\}\right)=\left\{g_{1}, g_{2}, g_{3}, g_{4}\right\}$. By Lemma 1.2.21, $M / g_{1}$ is 3 -connected. Thus, letting $M^{\prime}$ denote the matroid obtained via performing a $Y-\Delta$ on the triad $\left\{g_{1}, g_{2}, g_{3}\right\}$, we see that $M^{\prime} \backslash g_{1}$ is 3-connected and consequently that $M^{\prime} \backslash g_{1}, z$ is 3-connected too. This is a contradiction and so it must be the case that $|G|=7$. It is now clear that there exists $f_{0}, f_{6}$ such that $\left\{f_{0}, f_{1}, f_{2}\right\}$ and $\left\{f_{4}, f_{5}, f_{6}\right\}$ are triads of $M \backslash z$ and that $\left(f_{0}, f_{1}, \ldots, f_{5}, f_{6}\right)$ is a maximal fan as required.

2.1.1.2. The set $E(M)-(F \cup\{z\})$ contains a circuit of $M$.

Proof. Supposing otherwise, we have that $E(M)-(F \cup\{z\})$ is independent and exactly 3 -separating. Thus $E(M)-(F \cup\{z\})$ is a cosegment containing at least four elements and taking any $r \in E(M)-(F \cup\{z\})$ we have that $M / r$ is 3-connected. Let $\Gamma$ be any three element subset of $E(M)-(F \cup\{z\})$ such that $r \in \Gamma$ and let $M^{\prime}$ denote the matroid obtained via performing a $Y-\Delta$ exchange on $\Gamma$. Observe that $M^{\prime} \backslash r$ is 3-connected and therefore that $M^{\prime} \backslash r, z$ is also 3 -connected. We conclude that $E(M)-(F \cup\{z\})$ must contain a circuit.

Note that it follows from 2.1.1.2 that $E(M)-\mathrm{cl}_{M}^{*}(F \cup\{d\})$ is an exactly 3separating set which contains a circuit. Therefore we know that for $i \in\{6,0\}$, the partition $\left(\mathrm{cl}_{M}^{*}(F \cup\{d\})-\left\{f_{i}\right\},\left\{f_{i}\right\}, E(M)-\mathrm{cl}_{M}^{*}(F \cup\{d\})\right)$ is a cyclic 3-separation of $M$.

2.1.1.3. There exists $c \in E(M)-\mathrm{cl}_{M}^{*}(F \cup\{z\})$ such that $\left\{c, f_{0}, f_{6}, z\right\}$ is a 4-element circuit of $M$.

Proof. As the partitions $\left(\mathrm{cl}_{M}^{*}(F \cup\{d\})-\left\{f_{i}\right\},\left\{f_{i}\right\}, E(M)-\mathrm{cl}_{M}^{*}(F \cup\{d\})\right)$ are cyclic 3-separations of $M$ for $i \in\{6,0\}$, it follows from Bixby's Lemma that 
both $\operatorname{si}\left(M / f_{0}\right)$ and $\operatorname{si}\left(M / f_{6}\right)$ are 3-connected. Furthermore, by orthogonality, neither $f_{0}$ nor $f_{6}$ can be in a triangle of $M$ and so both $M / f_{0}$ and $M / f_{6}$ must be 3 -connected. As $M$ has no detachable pair, it must be that $\left\{f_{0}, f_{6}\right\}$ is contained in a 4-element circuit $C$. Clearly there exists some $c \in E(M)-$ $\mathrm{cl}_{M}^{*}(F \cup\{z\})$ such that $c \in C$, and now it follows from orthogonality with the two cocircuits $\left\{f_{0}, f_{1}, f_{2}, z\right\}$ and $\left\{f_{4}, f_{5}, f_{6}, z\right\}$ that the fourth element of $C$ must be $z$ as required.

2.1.1.4. There exists $w \in E(M)-\left\{f_{0}, f_{1}, f_{2}, f_{3}, f_{4}, f_{5}, f_{6}, z\right\}$ such that $\left\{w, f_{0}, f_{6}\right\}$ is a closed triad in $M$.

Proof. Consider the 3-connected matroid $M \backslash f_{5}$. The sequence $\left(f_{6}, z, f_{4}, f_{2}, f_{3}, f_{1}\right)$ is a fan in $M \backslash f_{5}$. By Lemma 1.1.7, as $M$ has no detachable pair, there must exist some $w$ such that $\left(f_{6}, z, f_{4}, f_{2}, f_{3}, f_{1}, w\right)$ is a fan of $M \backslash f_{5}$. Recall from 2.1.1.3 that $\left\{c, f_{0}, f_{6}, z\right\}$ is a 4 -element circuit of $M \backslash f_{5}$. By orthogonality with this circuit, it must be that $w \notin\left\{f_{0}, f_{6}\right\}$. It follows that $\left\{w, f_{0}, f_{6}\right\}$ must be a triad of $M \backslash f_{5}$ and therefore a triad of $M$. The fact that $\left\{w, f_{0}, f_{6}\right\}$ is a closed in $M$ follows easily from the fact that $\left\{w, f_{0}, f_{6}\right\}$ cannot be contained in a 4 -element circuit of $M$, together with the fact that there cannot exist a triangle of $M$ containing exactly two elements of $\left\{w, f_{0}, f_{6}\right\}$.

By 2.1.1.4 and Tutte's Triangle Lemma, there must exist some $t \in$ $\left\{w, f_{0}, f_{6}\right\}$ such that $M / t$ is 3 -connected. Letting $M^{\prime}$ denote the matroid obtained via performing a $Y-\Delta$ on the triad $\left\{w, f_{0}, f_{6}\right\}$, we now have that $M^{\prime} \backslash t$ is 3-connected, implying that $M^{\prime} \backslash t, z$ is 3 -connected as well. This is a contradiction. Thus, for the remainder of the proof, we may assume that $M$ contains no 4-element fans. Letting $T$ be some triangle of $M$, it now follows from Tutte's Triangle Lemma that there exists some $d \in T$ such that $M \backslash d$ is 3-connected. If $M \backslash d$ is not a wheel or a whirl, there must exist some $d^{\prime} \in E(M \backslash d)$ such that either $M \backslash d, d^{\prime}$ is 3-connected or $M \backslash d / d^{\prime}$ is 3connected. The former case gives a detachable pair as required. In the latter 
case, we know from Lemma 1.2.12 that $M^{\prime} / d$ is isomorphic to $M \backslash d$ where $M^{\prime}$ is the matroid obtained via performing a $\Delta-Y$ on the triangle $T$, and so it follows that $\left\{d, d^{\prime}\right\}$ is a detachable pair in $M^{\prime}$ provided $d^{\prime} \notin T$. In the case that $d^{\prime} \in T$, letting $d^{\prime \prime}=T-\left\{d, d^{\prime}\right\}$, we have that $\left\{d, d^{\prime \prime}\right\}$ is the requisite detachable pair. The only remaining possibility is that $M \backslash d$ is a wheel or a whirl. Now, as $M$ cannot have any 4-element fans, $d$ must block every triad of $M \backslash d$. This means that $d$ cannot be in the closure of the complement of any triad of $M \backslash d$. As every element of $M \backslash d$ is in a triad, it follows that the triangle $T$ of $M$ containing $d$ must intersect every triple that is a triad in $M \backslash d$. This is only possible if $M \backslash d$ is a small wheel or a small whirl; in particular with $|E(M \backslash d)| \leq 8$.

With Theorem 2.1.1 in hand, together with its dual, when searching for a detachable pair in some 3 -connected matroid $M$, we may henceforth assume that $M$ contains no 3 -element 3 -separating sets whatsoever.

\subsection{5-Point Planes}

In this section we deal with the case where $M$ has a restriction isomorphic to $U_{3,5}$.

Lemma 2.2.1. Let $M$ be a 3-connected matroid and let $C$ be a 4-element cocircuit of $M$. If there is a pair $\left\{c^{\prime}, c^{\prime \prime}\right\}$ of elements of $C$ such that neither $c^{\prime}$ nor $c^{\prime \prime}$ is contained in a triangle, then $C$ contains an element $c$ such that $M / c$ is 3-connected.

Proof. Let $C=\left\{c_{1}, c_{2}, c_{3}, c_{4}\right\}$ and suppose that $c_{1}$ is one of two elements which are not contained in triangles. If $M / c_{1}$ is not 3 -connected, then we have a vertical 3-separation $\left(X,\left\{c_{1}\right\}, Y\right)$ of $M$. We may assume that $c_{2} \in X$ and $c_{3}, c_{4} \in Y$. Suppose that $c_{2}$ is not in a triangle. If $X$ is a triad, then by Lemma 1.2.21, $M / c_{2}$ is 3-connected as required. If $X$ is not a triad, then $X$ is either a cosegment with at least four elements, or else $\left(X-\left\{c_{2}\right\}, Y \cup\left\{c_{1}\right\}\right)$ is a 
cyclic 2-separation of $M \backslash c_{2}$. The first case implies that $M / c_{2}$ is 3-connected, and the second case, by Bixby's Lemma, once again implies that $M / c_{2}$ is 3-connected. So $c_{2}$ must belong to some triangle $T$. As $C$ is a cocircuit, $T \cap\left(C-\left\{c_{2}\right\}\right) \neq \emptyset$, so we may assume that $c_{4}$ is the other element of $C$ which is not contained in a triangle and that $T=\left\{c_{2}, c_{3}, z\right\}$ for some $z \in E(M)-C$. As $c_{2} \notin \operatorname{cl}(Y),|T \cap X|=2$ and we see that $\left(X \cup\left\{c_{3}\right\},\left\{c_{1}\right\}, Y-\left\{c_{3}\right\}\right)$ is a vertical 3-separation of $M$. Again, if $Y-\left\{c_{3}\right\}$ is not a triad, then $\left(X \cup\left\{c_{1}, c_{3}\right\}, Y-\left\{c_{3}, c_{4}\right\}\right)$ is a cyclic 2-separation of $M \backslash c_{4}$ so that $M / c_{4}$ is 3-connected by Bixby's Lemma, while if $Y-\left\{c_{3}\right\}$ is indeed a triad, then $M / c_{4}$ is 3-connected by Lemma 1.2.21.

Lemma 2.2.2. Let $M$ be a 3-connected matroid and let $P$ be a set of elements of $M$ such that $M \mid P \cong U_{3,5}$. Assume that $\operatorname{cl}(P)$ contains no triangles and that $P$ contains no triads. If there is an element $p_{3} \in P$ such that $M \backslash p_{3}$ is not 3 -connected, then there is a partition $\left(\left\{p_{1}, p_{2}\right\},\left\{p_{4}, p_{5}\right\}\right)$ of $P-\left\{p_{3}\right\}$ such that $M \backslash p_{i}, p_{j}$ is 3-connected for all $i \in\{1,2\}$ and $j \in\{4,5\}$.

Proof. We first note that no element of $P$ is in a triad as such a triad would have to be contained in $P$ by orthogonality with the circuits within. Using this, together with the fact that $\mathrm{cl}(P)$ contains no triangles, we straightforwardly deduce that there is labelling $\left\{p_{1}, p_{2}, p_{3}, p_{4}, p_{5}\right\}$ of $P$ and a path $\left(A,\left\{p_{1}, p_{2}\right\},\left\{p_{3}\right\},\left\{p_{4}, p_{5}\right\}, B\right)$ of 3 -separations in $M$ such that $r(A), r(B) \geq 3$, $p_{1}, p_{2} \in \operatorname{cl}(A)$ and $p_{4}, p_{5} \in \operatorname{cl}(B)$. We have symmetry between $\left\{p_{1}, p_{2}\right\}$ and $\left\{p_{4}, p_{5}\right\}$, so it suffices to prove that $M \backslash p_{1}, p_{5}$ is 3 -connected. By Bixby's Lemma and the fact that $p_{1}$ is not in a triad of $M$, we see that $M \backslash p_{1}$ is 3connected. Again by Bixby's Lemma, we deduce that $M \backslash p_{1}, p_{5}$ is 3-connected unless it contains a series pair, that is, unless $M$ has a 4-element cocircuit $C$ containing both $p_{1}$ and $p_{5}$. By definition $C$ contains nothing in the span of its complement. Hence $C$ contains at least one element from each of $A$ and $B$, as well as at least three elements of $P$. But this implies that $|C| \geq 5$, and so we conclude that $M \backslash\left\{p_{1}, p_{5}\right\}$ is 3-connected as required.

The conditions that $P$ contains no triangle and that no element of $P$ is in a 
triad eliminate some degenerate outcomes whose analysis is not difficult. For our purposes the relatively weak conclusion of the next lemma is sufficient.

Lemma 2.2.3. Let $M$ be a 3-connected matroid with a set $P$ such that $M \mid P \cong U_{3,5}$. If $|E(M)| \geq 6$, then there exists distinct $p, p^{\prime} \in P$ such that both $M \backslash p$ and $M \backslash p^{\prime}$ are 3-connected.

Proof. Say that $P$ contains a triad $T$. Let $\left\{p, p^{\prime}\right\}=P-T$. It is easily checked that $M \backslash p$ and $M \backslash p^{\prime}$ are 3-connected. So assume that $P$ does not contain a triad. If $c \in P$ and $M \backslash c$ is not 3-connected, then we have a cyclic 3-separation $(X,\{c\}, Y)$ of $M$. If $r(M)=3$ then the lemma follows readily. So we may assume that $r(X) \geq 3$. Clearly $|X \cap P|=2=|Y \cap P|$. Let $X \cap P=\left\{p_{1}, p_{2}\right\}$. Then $p_{2} \in \operatorname{cl}(Y \cup\{c\})$ and $\left(X-\left\{p_{2}\right\},\{c\}, Y \cup\left\{p_{2}\right\}\right)$ is exactly 3 -separating, so $p_{2} \in \operatorname{cl}\left(X-\left\{p_{2}\right\}\right)$ hence $r\left(X-\left\{p_{2}\right\}\right) \geq 3$. Thus it follows that both $\left(X-\left\{p_{2}\right\},\left\{p_{2}\right\}, Y \cup\{c\}\right)$ and $\left(X-\left\{p_{1}\right\},\left\{p_{1}\right\}, Y \cup\{c\}\right)$ are vertical 3-separations of $M$. By Bixby's Lemma, both $M \backslash p_{1}$ and $M \backslash p_{2}$ are 3-connected.

We omit the easy proof of the next lemma.

Lemma 2.2.4. Let $M$ be a 3-connected matroid with a set $P$ such that $M \mid P \cong U_{3,5}$. If $z \in \operatorname{cl}(P)-P$, then $M \backslash z$ is 3-connected.

Lemma 2.2.5. Let $M$ be a 3-connected matroid with $|E(M)| \geq 7$ having a set $P$ such that $M \mid P \cong U_{3,5}$. Assume that $\mathrm{fcl}(P)$ contains no triangles or triads. Then $\mathrm{fcl}(P)$ contains a detachable pair.

Proof. Assume for a contradiction that $\mathrm{fcl}(P)$ contains no detachable pair. It follows from Lemmas 2.2.3 and 2.2.4 that $P$ is closed. By Lemma 2.2.2 $M \backslash p$ is 3-connected for all $p \in P$.

2.2.5.1. The lemma holds if $P$ is 3-separating.

Proof. If $P$ is 3 -separating, then $P$ is either a cocircuit or else properly contains a 4 -element cocircuit. If $P$ properly contains a 4 -element cocircuit $C^{*}$, 
let $c$ be some element of this cocircuit, let $P-C^{*}=\left\{p_{5}\right\}$, and note that $\left(C^{*},\left\{p_{5}\right\}, E(M)-P\right)$ is a vertical 3-separation of $M$. By Bixby's Lemma, $\operatorname{co}\left(M \backslash c, p_{5}\right)$ is 3 -connected, so the result holds unless there is some triad $\left\{p, p_{5}, z\right\}$ in $M \backslash c$ where $p \in P$ and $z \in E(M)-P$. In the case where $P$ is itself a cocircuit, take any $p_{5} \in P$ and let $\left\{p_{1}, p_{2}, p_{3}\right\}=P-\left\{c, p_{5}\right\}$. Suppose that there is some 2-separation $(X, Y)$ of $M \backslash c, p_{5}$. We may assume that $p_{1}, p_{2} \in X$. Now $\left\{p_{1}, p_{2}, p_{3}\right\}$ is a triad of $M \backslash c, p_{5}$, so provided $Y$ is not a series pair, $\left(X \cup\left\{p_{3}\right\}, Y-\left\{p_{3}\right\}\right)$ is also a 2-separation of $M \backslash c, p_{5}$. But then $p_{5} \in \operatorname{cl}_{M \backslash c}\left(X \cup\left\{p_{3}\right\}\right)$ so that $\left(X \cup\left\{p_{3}, p_{5}\right\}, Y-\left\{p_{3}\right\}\right)$ is a contradictory 2separation of the 3-connected matroid $M \backslash c$. We are left to conclude that $Y$ is a series pair and that, as in the case where $P$ contains a 4 -element cocircuit, there is some triad $\left\{p, p_{5}, z\right\}$ in $M \backslash c$ with $p \in P$ and $z \in E(M)-P$. We now deal with both of these cases together. Certainly $M / z$ is 3 -connected, as is $M / p_{i}$ for all $p_{i} \in\left(P-\left\{p_{5}\right\}\right)$. Because $\mathrm{fcl}(P)$ contains no detachable pair nor any triangles, it is readily deduced that $M$ must contain a 4-element circuit $C$ where $C=\left\{z, p^{\prime}, p^{\prime \prime}, d\right\}$ for some $p^{\prime}, p^{\prime \prime} \in\left(P-\left\{p_{5}\right\}\right)$ and $d \in(E(M)-(P \cup\{z\}))$. As $\mathrm{fcl}(P)$ contains no triads nor triangles, $(P \cup\{z\},\{d\}, E(M)-(P \cup\{z, d\}))$ is a vertical 3-separation of $M$ and $M \backslash d$ is 3-connected. It now follows from Lemma 2.2.3 that $M$ has a detachable pair.

Thus we may assume for the remainder of the proof that $\lambda(P)=3$.

2.2.5.2. One of the following holds.

(i) There are elements $u$ and $v$ of $M$ and a labelling $\left\{p_{1}, p_{2}, p_{3}, p_{4}, p_{5}\right\}$ of $P$ such that $\left\{p_{1}, p_{2}, p_{3}, u\right\}$ and $\left\{p_{3}, p_{4}, p_{5}, v\right\}$ are cocircuits of $M$.

(ii) There is an element $p \in P$ such that there are two elements of $P-\{p\}$ that are not contained in triads of $M \backslash p$.

Proof. Assume that (i) does not hold. By 2.2.5.1, each 4-element cocircuit that intersects $P$ contains exactly three elements of $P$. If there is an element 
$p \in P$ that does not belong to any 4-element cocircuit, then $M \backslash p$ clearly satisfies (ii). Assume that every element of $P$ belongs to at least one 4-element cocircuit. Call the triples of $P$ that are contained in 4-element cocircuits cocircuit triples. Without loss of generality, $\left\{p_{1}, p_{2}, p_{3}\right\}$ is such a triple. Consider $p_{4}$. If there is a cocircuit triple containing $p_{4}$ that also contains $p_{5}$, then the claim clearly holds. Thus, we may assume up to labels, that $\left\{p_{2}, p_{3}, p_{4}\right\}$ is a cocircuit triple. Consider $p_{5}$. The cocircuit triples containing $p_{5}$ do not contain $p_{4}$. The only possible triple that can be constructed without satisfying (i) is $\left\{p_{2}, p_{3}, p_{5}\right\}$. It is easily checked that if there are any more cocircuit triples, then (i) holds. Now consider $M \backslash p_{1}$. Neither $p_{4}$ nor $p_{5}$ belong to triads in $M \backslash p_{1}$, so (ii) holds in this case.

If 2.2.5.2 (ii) holds, then it follows from the dual of Lemma 2.2.1 that $M$ must contain a detachable pair. This leaves us to resolve situation (i) of 2.2.5.2. If $u \in P$, then we see that $\lambda(P)=2$, contradicting the fact that $\lambda(P)=3$. Hence $\{u, v\} \cap P=\emptyset$. If $u=v$, then $P$ must again contain a cocircuit and again we contradict the fact that $\lambda(P)=3$. So $u \neq v$.

2.2.5.3. $M / u$ is 3 -connected.

Proof. Say $(X, Y)$ is a 2-separation of $M / u$ where neither $X$ nor $Y$ is a parallel class. We may assume that $|P \cap X| \geq 3$. As $u \notin \mathrm{cl}_{M}(P)$, we see that $(M / u) \mid P \cong U_{3,5}$. Note that $(\operatorname{cl}(X), Y-\operatorname{cl}(X))$ is also a 2-separation of $M / u$ where neither $\operatorname{cl}(X)$ nor $Y-\operatorname{cl}(X)$ is a parallel class. Hence we may assume that $X \subseteq P$. But $u \in \mathrm{cl}_{M}^{*}(P)$, so $(X \cup\{u\}, Y)$ is a 2-separation of $M$, contradicting the fact that $M$ is 3 -connected. Hence $M / u$ is 3-connected up to parallel sets. If $T$ is a triangle containing $u$, then $T$ contains another element of the cocircuit $\left\{p_{1}, p_{2}, p_{3}, u\right\}$, that is, $T$ contains at least two elements of $\mathrm{fcl}_{M}(P)$ so that $T \subseteq \mathrm{fcl}_{M}(P)$. Hence $u$ does not belong to a triangle and we conclude that $M / u$ is indeed 3-connected.

2.2.5.4. $M \backslash u$ and $M \backslash v$ are not 3-connected. 
Proof. Assume that $M \backslash u$ is 3-connected. By Lemma 2.2.3, there is an element $p \in P$ such that $M \backslash u, p$ is 3-connected, contradicting the assumption that $\operatorname{fcl}_{M}(P)$ has no detachable pair.

2.2.5.5. $v \notin \mathrm{cl}_{M / u}(P)$.

Proof. Otherwise, by Lemma 2.2.4 M/u\v is 3-connected. In such a situation, either $M \backslash v$ is 3 -connected or $u$ and $v$ belong to a triad $T$ of $M$. In the latter case, as $u, v \in \mathrm{cl}^{*}(P)$ we see that $T \subseteq \mathrm{fcl}(P)$ contradicting the fact that there are no triads in this set. The former case contradicts 2.2.5.4.

2.2.5.6. $M / u, v$ is 3 -connected up to parallel sets.

Proof. Say $(X, Y)$ is a 2-separation of $M / u, v$ where neither $X$ nor $Y$ is a parallel class. We may assume that $|P \cap X| \geq 3$ and that $X$ is closed. By 2.2.5.5, $v \notin \operatorname{cl}_{M / u}(P)$. Hence $(M / u, v) \mid P \cong U_{3,5}$ and $P \subseteq X$. But $u, v \in \mathrm{cl}_{M}^{*}(P)$ so that $u, v \in \mathrm{cl}_{M}^{*}(X)$. So $(X \cup\{u, v\}, Y)$ is a 2-separation of $M$, contradicting the fact that $M$ is 3 -connected. Thus $M / u, v$ has no non-trivial 2-separations and the claim follows.

If $M / u, v$ is 3 -connected, then $\{u, v\}$ is a detachable pair, so we may assume that $M / u, v$ is not 3-connected. By 2.2.5.6, the element $v$ is in a triangle $T$ of $M / u$. The triangle $T$ contains at least two elements of the cocircuit $\left\{p_{3}, p_{4}, p_{5}, v\right\}$ of $M / u$. Thus $T=\{p, v, z\}$ for some $p \in P$. Clearly $T \subseteq \mathrm{fcl}_{M}(P)$. Moreover $z \notin P$, as otherwise $v \in \mathrm{cl}_{M / u}(P)$ contradicting 2.2.5.5. The triangle $T$ is not contained in a 4-element fan of $M / u$ as such a fan would imply the existence of a triad in $\operatorname{fcl}_{M}(P)$. Hence either $M / u \backslash v$ or $M / u \backslash z$ is 3-connected. As $\mathrm{fcl}_{M}(P)$ contains no triads we deduce that $M \backslash v$ or $M \backslash z$ is 3-connected. We already know that $M \backslash v$ is not 3-connected, but $z$ is just as problematic for the same reason, as, in this case too we deduce from Lemma 2.2.3 that there is an element $p \in P$ such that $M \backslash z, p$ is 3-connected. Again this contradicts the assumption that there is no detachable pair in $\mathrm{fcl}_{M}(P)$. The lemma follows from this final contradiction. 


\subsection{Specific Triad Situations}

The next two lemmas deal with two very specific situations that arise in our later analysis. These two situations are largely isolated from the bigger picture contained in subsequent chapters and so we shall deal with them here.

Lemma 2.3.1. Let $M$ be a 3-connected matroid with an element d such that $M \backslash d$ is 3-connected. Assume that $M \backslash d$ has a set $Z=\left\{s_{1}, s_{2}, t_{1}, t_{2}, u\right\}$ such that the following hold:

(i) $\mathrm{fcl}_{M}(Z \cup\{d\})$ contains no triangles or triads,

(ii) $Z$ is closed in $M \backslash d$,

(iii) $\lambda_{M \backslash d}(Z)=2$,

(iv) $\left\{s_{1}, s_{2}, u\right\}$ and $\left\{t_{1}, t_{2}, u\right\}$ are triads of $M \backslash d$, and

(v) $Z$ contains no 4-element circuits.

Then $\mathrm{fcl}_{M}(Z \cup\{d\})$ contains a detachable pair.

Proof. Assume that $\mathrm{fcl}_{M}(Z \cup\{d\})$ does not contain a detachable pair. Let $Y=E(M)-(Z \cup\{d\})$.

2.3.1.1. If $s \in\left\{s_{1}, s_{2}\right\}$ and $t \in\left\{t_{1}, t_{2}, u\right\}$, then $M \backslash d / s, t$ is 3-connected.

Proof. Take any $s \in\left\{s_{1}, s_{2}\right\}, t \in\left\{t_{1}, t_{2}\right\}$. The fact that $M \backslash d / s$ is 3 -connected follows immediately from Lemma 1.2.14. In the same way, $M \backslash d / s, t$ must now be 3-connected unless $t$ is contained in a triangle of $M \backslash d / s$. This would assert the existence of a 4 -element circuit $C$ containing $\{s, t\}$ in the matroid $M \backslash d$. By orthogonality with the triads of $Z$, we must have $|C \cap Z| \geq 3$, and as $Z$ is closed in $M \backslash d$, we see that $C \subseteq Z$. This contradicts property (v).

2.3.1.2. $d$ blocks $Z$. 
Proof. Assume otherwise. Then, $d \in \operatorname{cl}(Z)$. By 2.3.1.1, the assumption that $Z$ is closed in $M \backslash d$ and the assumption that $\operatorname{fcl}(Z \cup\{d\})$ has no detachable pair, we see that every element of $Z-\left\{s_{1}\right\}$ is in a triangle with $d$ in $M / s_{1}$. These triangles must come from 4-element circuits of $M$ containing $d$. It follows that, up to symmetry, there are two cases. Either (a) $\left\{s_{1}, d, s_{2}, u\right\}$ and $\left\{s_{1}, d, t_{1}, t_{2}\right\}$ are circuits of $M$ or (b) $\left\{s_{1}, d, s_{2}, t_{2}\right\}$ and $\left\{s_{1}, d, u, t_{1}\right\}$ are circuits of $M$. Consider case (a). Replacing $s_{1}$ by $s_{2}$ we see that there are analogous 4-element circuits containing $\left\{s_{2}, d\right\}$. We already know that $\left\{s_{1}, d, s_{2}, u\right\}$ is one, so the other must be $\left\{s_{2}, d, t_{1}, t_{2}\right\}$. But then $s_{2} \in \operatorname{cl}\left(\left\{s_{1}, d, t_{1}, t_{2}\right\}\right)$. As there are no triangles in $Z \cup\{d\}$, we see that $\left\{s_{1}, s_{2}, t_{1}, t_{2}\right\}$ is a circuit, contradicting the assumption that $Z$ contained no 4-element circuits. Case (b) is essentially the same.

It is now a straightforward consequence of 2.3.1.1, 2.3.1.2 and the assumption that $\operatorname{fcl}_{M}(Z \cup\{d\})$ contains no detachable pairs that there are elements $v_{1}, v_{2}, v_{3}, w_{1}, w_{2}, w_{3} \in Y \cap \mathrm{cl}_{M}(Z \cup\{d\})$ such that $\left\{s_{1}, t_{1}, d, v_{1}\right\}$, $\left\{s_{1}, t_{2}, d, v_{2}\right\},\left\{s_{1}, u, d, v_{3}\right\},\left\{s_{2}, t_{1}, d, w_{1}\right\},\left\{s_{2}, t_{2}, d, w_{2}\right\}$, and $\left\{s_{2}, u, d, w_{3}\right\}$ are circuits. If $v_{i}=v_{j}$ for some pair of distinct indices, then we obtain the contradiction that $Z$ contains a 4 -element circuit. Thus the elements $v_{1}, v_{2}$ and $v_{3}$ are distinct, as are the elements $w_{1}, w_{2}$ and $w_{3}$. Say $v_{i}=w_{j}$ for some $i, j \in\{1,2,3\}$. Then we have a circuit $\left\{s_{1}, \alpha, d, v_{i}\right\}$ and a circuit $\left\{s_{2}, \beta, d, v_{i}\right\}$, where $\alpha, \beta \in\left\{t_{1}, t_{2}, u\right\}$. But now $\left\{s_{1}, s_{2}, \alpha, \beta, v_{i}\right\}$ contains a circuit. But $Z$ is closed in $M \backslash d$, so $v_{i} \notin \operatorname{cl}\left(\left\{s_{1}, s_{2}, \alpha, \beta\right\}\right)$. Hence $\left\{s_{1}, s_{2}, \alpha, \beta\right\}$ contains a circuit of $M$, contradicting the fact that $Z$ contains no circuits of size 3 or 4 . Hence the elements $v_{1}, v_{2}, v_{3}, w_{1}, w_{2}, w_{3}$ are all distinct. As $Y \cap \mathrm{cl}_{M}(Z \cup\{d\})$ has rank at most 3 , we see that $M \mid\left\{v_{1}, v_{2}, v_{3}, w_{1}, w_{2}\right\} \cong U_{3,5}$. By Lemma 2.2.5, fcl $\left(\left\{v_{1}, v_{2}, v_{3}, w_{1}, w_{2}\right\}\right)$ contains a detachable pair. But $\operatorname{fcl}\left(\left\{v_{1}, v_{2}, v_{3}, w_{1}, w_{2}\right\}\right) \subseteq \mathrm{fcl}(Z)$ and the lemma follows.

Lemma 2.3.2. Let $M$ be a 3-connected matroid with an element d such that $M \backslash d$ is 3-connected. Let $(S, T, Z)$ be a paddle in $M \backslash d$ such that the following hold: 
(a) $S$ and $T$ are triads of $M \backslash d$,

(b) $d$ blocks both $S$ and $T$ in $M$,

(c) $\operatorname{fcl}(S \cup T \cup\{d\})$ contains no triads or triangles, and

(d) $\operatorname{fcl}_{M \backslash d}(S \cup T) \neq E(M \backslash d)$.

Then either:

(i) there exists $c_{1}, c_{2} \in \mathrm{cl}_{M \backslash d}^{*}(S \cup T)$ such that $M / c_{1}, c_{2}$ is 3-connected, or

(ii) there exists $m \in \mathrm{cl}_{M \backslash d}(S)$ such that $M \backslash d, m$ is 3-connected, or

(iii) there exists $s \in S, t \in T$ such that $M \backslash s, t$ is 3-connected.

Proof. If there exists some $m \in\left(\operatorname{cl}_{M \backslash d}(S)-S\right)$, then by Bixby's Lemma, $\operatorname{co}(M \backslash d, m)$ is 3-connected. If $m$ is contained in a triad of $M \backslash d$, then this triad must meet both $S \cup T$ and $E(M \backslash d)-(S \cup T)$. But such a situation contradicts property (c) and so $M \backslash d, m$ is 3-connected giving (ii). So assume henceforth that $S$, and consequently $S \cup T$, is closed. Let $M^{\prime}=M \backslash d$. Suppose now that there exists distinct $c_{1}, c_{2} \in \mathrm{cl}_{M^{\prime}}^{*}(S \cup T)$. By Bixby's Lemma and property (c), $\operatorname{si}\left(M^{\prime} / c_{1}, c_{2}\right)$ is 3-connected. If $C^{\prime}$ is a circuit of $M^{\prime}$ of size at most four containing $\left\{c_{1}, c_{2}\right\}$, then $C^{\prime}$ must contain exactly one element of $S \cup T$. But then $C^{\prime}$ intersects one of the triads $S$ or $T$ of $M^{\prime}$ at one point, which contradicts orthogonality. Thus $M^{\prime} / c_{1}, c_{2}$ is 3-connected. If therefore $M / c_{1}, c_{2}$ fails to be 3 -connected, then $M$ must contain a circuit $C=\left\{c_{1}, c_{2}, d, \alpha\right\}$. As $S \cup\{d\}$ is a cocircuit in $M$, it must be that $\alpha \in S$. But then $C \cap(T \cup\{d\})=\{d\}$, again contradicting orthogonality. So (i) holds in this situation. We may assume then that $\left|\mathrm{cl}_{M^{\prime}}^{*}(S \cup T)-(S \cup T)\right| \leq 1$.

2.3.2.1. If $s \in S$, then there exists at most one element $t \in T$ such that $(S \cup T)-\{s, t\}$ is a circuit in $M^{\prime}$.

Proof. This follows straightforwardly from the fact that $S$ and $T$ are triads of $M^{\prime}$ with $r_{M^{\prime}}(S \cup T)=4$. 
Let $S=\left\{s_{1}, s_{2}, s_{3}\right\}$ and $T=\left\{t_{1}, t_{2}, t_{3}\right\}$. By 2.3.2.1 we may assume that $\left\{s_{2}, s_{3}, t_{2}, t_{3}\right\}$ is independent.

2.3.2.2. $M^{\prime} \backslash s_{1}, t_{1}$ is connected.

Proof. Suppose that $(P, Q)$ is a separation of $M^{\prime} \backslash s_{1}, t_{1}$. As $\left\{s_{2}, s_{3}\right\}$ is a cocircuit of $M^{\prime} \backslash s_{1}, t_{1}$, we may assume that $\left\{s_{2}, s_{3}\right\} \subseteq P$. Likewise, we may assume that $\left\{t_{2}, t_{3}\right\}$ is contained in either $P$ or $Q$. If then $\left\{t_{2}, t_{3}\right\} \subseteq P$ it would follow that $(P, Q)$ is a contradictory separation in the 3-connected matroid $M^{\prime}$ as $\left\{s_{1}, t_{1}\right\} \subseteq \operatorname{cl}_{M^{\prime}}\left(\left\{s_{2}, s_{3}, t_{2}, t_{3}\right\}\right)$. Therefore we may assume that $\left\{s_{2}, s_{3}\right\} \subseteq P$ and $\left\{t_{2}, t_{3}\right\} \subseteq Q$ with $P \cap Z \neq \emptyset \neq Q \cap Z$. Let $\lambda=\lambda_{M^{\prime} \backslash s_{1}, t_{1}}$. By submodularity of $\lambda$ we have

$$
\begin{array}{r}
\lambda(P \cap Z)+\lambda(Q \cap Z) \\
\leq \lambda(P)+\lambda(Z)-\lambda(P \cup Z)+\lambda(Q)+\lambda(Z)-\lambda(Q \cup Z) \\
=\lambda(Z)+\lambda(Z)-\lambda(Q \cup Z)-\lambda(P \cup Z) \\
=4-\lambda\left(\left\{s_{2}, s_{3}\right\}\right)-\lambda\left(\left\{t_{2}, t_{3}\right\}\right)=2 .
\end{array}
$$

If either $\lambda(P \cap Z)=0$ or $\lambda(Q \cap Z)=0$ then, as $\left\{s_{1}, t_{1}\right\} \in \operatorname{cl}_{M^{\prime}}\left(\left\{s_{2}, s_{3}, t_{2}, t_{3}\right\}\right)$, we obtain a separation in $M^{\prime}$. Thus $\lambda(P \cap Z)=1=\lambda(Q \cap Z)$. As $|Z| \geq 3$, we may assume without loss of generality that $|P \cap Z| \geq 2$. But now it follows that $\left(P \cap Z, E\left(M^{\prime}\right)-(P \cap Z)\right)$ is a 2-separation in $M^{\prime}$.

Suppose that $c \in Z \cap \operatorname{cl}_{M^{\prime} \backslash s_{1}, t_{2}}^{*}\left(\left\{s_{2}, s_{3}\right\}\right)$ and let $Z^{\prime}=Z-\{c\}$.

2.3.2.3. $r\left(Z^{\prime} \cup\left\{t_{2}, t_{3}\right\}\right)=r\left(Z^{\prime}\right)+1$ and $r\left(Z^{\prime} \cup\left\{s_{2}, s_{3}\right\}\right)=r\left(Z^{\prime}\right)+2$.

Proof. The fact that $r\left(Z^{\prime} \cup\left\{t_{2}, t_{3}\right\}\right)=r(M)-2=r\left(Z^{\prime}\right)+1$ follows immediately from the fact that $\left\{s_{2}, s_{3}, c\right\}$ is a series class in $M^{\prime} \backslash s_{1}, t_{1}$. If then $r\left(Z^{\prime} \cup\left\{s_{1}, s_{2}\right\}\right)<r\left(Z^{\prime}\right)+2$, it must be that

$$
r\left(Z^{\prime} \cup\left\{s_{1}, s_{2}, t_{1}, t_{2}\right\}\right) \leq r\left(Z^{\prime}\right)+2<r(M) .
$$

But $E\left(M^{\prime} \backslash s_{1}, t_{1}\right)-\left(Z^{\prime} \cup\left\{s_{1}, s_{2}, t_{1}, t_{2}\right\}\right)=\{c\}$ so that $c$ must be a coloop of $M^{\prime} \backslash s_{1}, t_{1}$. However we know from 2.3.2.2 that $M^{\prime} \backslash s_{1}, t_{1}$ is connected and so we have a contradiction. 
2.3.2.4. $r\left(Z^{\prime} \cup\left\{t_{1}, t_{2}\right\}\right)=r\left(Z^{\prime} \cup\left\{t_{1}, t_{3}\right\}\right)=r\left(Z^{\prime}\right)+2$.

Proof. Suppose that $r\left(Z^{\prime} \cup\left\{t_{1}, t_{2}\right\}\right)=r\left(Z^{\prime}\right)+1$. Then $t_{2} \in \operatorname{cl}\left(Z^{\prime} \cup\left\{t_{1}\right\}\right)$ and by 2.3.2.3, $r\left(Z^{\prime} \cup T\right)=r\left(Z^{\prime}\right)+1$. As $r\left(Z^{\prime} \cup T \cup\{c\}\right)=r\left(Z^{\prime}\right)+2, c \notin \operatorname{cl}_{M^{\prime}}\left(Z^{\prime} \cup T\right)$ implying that $c \in \mathrm{cl}_{M^{\prime}}^{*}(S)$. But this contradicts Lemma 1.2.9.

Now combining what we have learned from 2.3.2.1, 2.3.2.3, and 2.3.2.4, it follows that by potentially relabelling, we may assume henceforth that $\left\{s_{2}, s_{3}, t_{2}, t_{3}\right\}$ is independent and that neither $\left\{s_{2}, s_{3}\right\}$ nor $\left\{t_{2}, t_{3}\right\}$ is contained in a three-element 2-separating set in $M^{\prime} \backslash s_{1}, t_{1}$.

2.3.2.5. If $(P, Q)$ is a 2-separation of $M^{\prime} \backslash s_{1}, t_{1}$, then, up to labelling, $\left\{s_{2}, s_{3}\right\} \subseteq P$ and $\left\{t_{2}, t_{3}\right\} \subseteq Q$.

Proof. Assume otherwise. Up to labelling we have that either $s_{2} \in P$ and $\left\{s_{3}, t_{2}, t_{3}\right\} \subseteq Q$ or $\left\{s_{2}, t_{2}\right\} \subseteq P$ and $\left\{s_{3}, t_{3}\right\} \subseteq Q$. Suppose the former of these holds. If $|P|=2$, then $P \cup\left\{s_{3}\right\}$ is a three-element series class of $M^{\prime} \backslash s_{1}, t_{1}$, contradicting what we have just shown. So $|P| \geq 3$; giving in particular that $|P \cap Z| \geq 2$. We shall look to show that $P \cap Z$ is 2-separating in $M^{\prime} \backslash s_{1}, t_{1}$, which in turn would imply that $P \cap Z$ is 2 -separating in the 3-connected matroid $M^{\prime}$ as $\left\{s_{1}, t_{1}\right\} \subseteq \operatorname{cl}_{M^{\prime}}\left(\left\{s_{2}, s_{3}, t_{2}, t_{3}\right\}\right)$. Let $\lambda^{\prime}=\lambda_{M^{\prime} \backslash s_{1}, t_{1}}$. By submodularity of the connectivity function,

$$
\begin{array}{r}
\lambda^{\prime}\left(Q \cup\left(E\left(M^{\prime} \backslash s_{1}, t_{1}\right)-Z\right)\right) \\
\leq \lambda^{\prime}(Q)+\lambda^{\prime}\left(\left\{s_{2}, s_{3}, t_{2}, t_{3}\right\}\right)-\lambda^{\prime}\left(Q \cap\left(E\left(M^{\prime} \backslash s_{1}, t_{1}\right)-Z\right)\right) \\
\leq 1+2-\lambda^{\prime}\left(\left\{s_{3}, t_{2}, t_{3}\right\}\right) .
\end{array}
$$

As we have shown, $\left\{s_{3}, t_{2}, t_{3}\right\}$ is not 2-separating in $M^{\prime} \backslash s_{1}, t_{1}$ and so $\lambda^{\prime}(Q \cup$ $\left.\left\{s_{2}, s_{3}, t_{2}, t_{3}\right\}\right) \leq 1+2-2=1$. Equivalently, $\lambda^{\prime}(P \cap Z)=1$, and we arrive at our contradiction. Now suppose that the other possibility holds so that $\left\{s_{2}, t_{2}\right\} \subseteq P$ and $\left\{s_{3}, t_{3}\right\} \subseteq Q$. As $E\left(M^{\prime}\right)-(S \cup T)$ is non-sequential, $|Z| \geq 4$, and so we may assume that $|P \cap Z| \geq 2$. It now follows that, as $t_{2} \in \operatorname{cl}_{M^{\prime} \backslash s_{1}, t_{1}}^{*}\left(\left\{t_{3}\right\}\right)$, the partition $\left(P-\left\{t_{2}\right\}, Q \cup\left\{t_{2}\right\}\right)$ is a 2-separation 
of $M^{\prime} \backslash s_{1}, t_{1}$. But now we are reduced to the previous case. The result follows.

Suppose that $(V, W)$ is a 2-separation of $M \backslash s_{1}, t_{1}, d$ with $W \cap Z \neq \emptyset$. By 2.3.2.5, $\left\{s_{2}, s_{3}\right\} \subseteq V$ and $\left\{t_{2}, t_{3}\right\} \subseteq W$. As $T \cup\{d\}$ is a 4-element cocircuit in $M$ it follows that $\left\{t_{2}, t_{3}, d\right\}$ is a triad of $M \backslash s_{1}, t_{1}$. Hence $d \notin \mathrm{cl}_{M \backslash s_{1}, t_{1}}(V)$, and we conclude that $(V \cup\{d\}, W)$ is an exactly 3-separating partition of the matroid $M \backslash s_{1}, t_{1}$. As $(V, W)$ was an arbitrarily chosen 2-separation the result follows.

\section{$2.4 \quad$ Flans}

Recall that flans were defined at 1.1.8. Flans arise throughout this work. They can be considered as underlying structures of problematic 3-separating sets which yield no detachable pair. In particular, both partial spider-like 3separators and twisted 3-separators are single-element extensions of a generic 5-element flan. This fact is elucidated in more detail in Lemma 3.1.3. Our interest here is in flans that contain no triangles. The following lemma is immediate.

Lemma 2.4.1. Let $F$ be a flan of the 3-connected matroid $M$.

(i) If $3 \leq i \leq n$ is odd, then $f_{i} \in \mathrm{cl}^{*}\left(\left\{f_{1}, f_{2}, \ldots, f_{i-1}\right\}\right)$.

(ii) $F$ is a sequential 3-separating set with sequential ordering $\left(f_{1}, f_{2}, \ldots, f_{n}\right)$.

Lemma 2.4.2. Let $F=\left(f_{1}, f_{2}, \ldots, f_{n}\right)$ be a maximal flan of the 3 -connected matroid $M$, where $n \geq 4$ and $F \neq E(M)$. If $n$ is even, then $M \backslash f_{n}$ is 3-connected.

Proof. If $E(M)-F=\left\{f_{n+1}\right\}$ is a singleton, then it follows that $\left\{f_{n-1}, f_{n}, f_{n+1}\right\}$ is a triad of $M$, contradicting the maximality of $F$. So 
$|E(M)-F| \geq 2$. If $r(E(M)-F) \geq 3$, then $\left(F-\left\{f_{n}\right\},\left\{f_{n}\right\}, E(M)-F\right)$ is a vertical 3-separation of $M$, and so, by Bixby's Lemma, $\operatorname{co}\left(M \backslash f_{n}\right)$ is 3connected. In this case, $M \backslash f_{n}$ is 3-connected unless $f_{n}$ is contained in a triad $T$. But in such a situation, $\left|T \cap\left(F-\left\{f_{n}\right\}\right)\right|=1=|T \cap(E(M)-F)|$, and by the construction of the flan $F$ it follows that $\left(f_{1}, f_{2}, \ldots, f_{n}, t\right)$ is a flan of $M$ where $\{t\}=T \cap(E(M)-F)$; again contradicting the maximality of $F$. Otherwise, $r(E(M)-F)=2$. Now, if $|E(M)-F|=3$, then $(E(M)-F) \cup\left\{f_{n}\right\}$ is a 4-element rank-2 set and $M \backslash f_{n}$ is obviously 3-connected. The only remaining possibility is that $(E(M)-F) \cup\left\{f_{n}\right\}$ is a triangle. It is clear that $f_{n}$ cannot belong to any triads of $M$, and so by Lemma 1.2.14, $M \backslash f_{n}$ must be 3-connected.

Lemma 2.4.3. Let $F=\left(f_{1}, f_{2}, \ldots, f_{n}\right)$ be a maximal flan of the 3 -connected matroid $M$, where $F \neq E(M)$ and $n \geq 5$ is odd. If $F$ contains no triangles then the following hold.

(i) If $i \in\{1,3,5, \ldots, n-2\}$, then $M / f_{i}, f_{n}$ is 3-connected.

(ii) $M / f_{2}, f_{n}$ is 3-connected.

Proof. Suppose that $\left(F-\left\{f_{n}\right\},\left\{f_{n}\right\}, E(M)-F\right)$ is not a cyclic 3-separation of $M$. If we have $|E(M)-F|<3$, it follows that $\operatorname{cl}(F)=E(M)$; contradicting the maximality of $F$. So $(E(M)-F) \cup\left\{f_{n}\right\}$ is a cosegment containing at least four elements and so $M / f_{n}$ is 3-connected. It now follows from Bixby's Lemma and the fact that $\operatorname{cl}(F)-F=\emptyset$ that $M / f_{n}, f_{i}$ is 3-connected for all odd $i \in\{1, \ldots, n-2\}$ as well as for $f_{2}$. Otherwise, $\left(F-\left\{f_{n}\right\},\left\{f_{n}\right\}, E(M)-F\right)$ is cyclic and so $\operatorname{si}\left(M / f_{n}\right)$ is 3-connected; again implying that $M / f_{n}$ and $M / f_{n}, f_{i}$ is 3 -connected for all odd $i \in\{1, \ldots, n-2\}$ as well as for $f_{2}$.

Lemma 2.4.4. Let $M$ be a 3-connected matroid and let $d$ be an element of $M$ such that $M \backslash d$ is 3-connected. Let $F=\left(f_{1}, f_{2}, \ldots, f_{n}\right)$ be a maximal flan of $M \backslash d$ with at least six elements where $F \cup\{d\} \neq E(M)$. Assume that $F \cup\{d\}$ contains no triangles or triads. Then $F \cup\{d\}$ contains a detachable pair. 
Proof. Assume that there is no detachable pair contained in $F \cup\{d\}$. Suppose that $n$ is even. Then, by Lemma 2.4.2, $M \backslash d, f_{n}$ is 3 -connected provided $M \backslash d$ is not a wheel or a whirl. In the exceptional case, we contradict our assumption that $(F \cup\{d\}) \neq E(M)$. Thus $\left\{d, f_{n}\right\}$ is a detachable pair and so we may assume henceforth that $n$ is odd. By Bixby's Lemma, $M \backslash d / f_{n}$ is 3-connected. Now if $d$ is in a parallel pair in $M / f_{n}$, then, as $F \cup\{d\}$ contains no triangles of $M$, the other element of this parallel pair belongs to $E(M)-(F \cup\{d\})$. But then $d$ does not block the triads of $F-\left\{f_{n}\right\}$ in $M / f_{n}$. Hence $M / f_{n}$ is 3 -connected.

2.4.4.1. There is a set $T=\left\{t_{1}, t_{2}, t_{3}\right\}$ such that $\left\{f_{1}, d, t_{1}\right\},\left\{f_{2}, d, t_{2}\right\}$ and $\left\{f_{3}, d, t_{3}\right\}$ are triangles in $M / f_{n}$.

Proof. By Lemma 2.4.3 $M \backslash d / f_{1}, f_{n}, M \backslash d / f_{2}, f_{n}$ and $M \backslash d / f_{3}, f_{n}$ are all 3connected. Also $M / f_{n}$ is 3 -connected. If we do not have the triangles of the claim, then we have a detachable pair in $F \cup\{d\}$.

2.4.4.2. $\left\{t_{1}, t_{2}, t_{3}\right\} \subseteq F-\left\{f_{n}\right\}$.

Proof. Say $t_{1} \notin F-\left\{f_{n}\right\}$. Then the triad $\left\{f_{3}, f_{4}, f_{5}\right\}$ of $M \backslash d / f_{n}$ is not blocked by $d$. Hence $t_{1} \in F-\left\{f_{n}\right\}$, so that $d \in \mathrm{cl}_{M / f_{n}}\left(F-\left\{f_{n}\right\}\right)$. Therefore we also have $\left\{t_{1}, t_{2}, t_{3}\right\} \subseteq(F-\{d\})$.

2.4.4.3. $\left\{t_{1}, t_{2}, t_{3}\right\} \cap\left\{f_{1}, f_{2}, f_{3}\right\}=\emptyset$.

Proof. Suppose otherwise. Then $d \in \operatorname{cl}_{M / f_{n}}\left(\left\{f_{1}, f_{2}, f_{3}\right\}\right)$. As $f_{5} \notin$ $\operatorname{cl}_{M / f_{n}}\left(\left\{f_{1}, f_{2}, f_{3}, f_{4}, d\right\}\right) \subseteq \operatorname{cl}_{M / f_{n}}\left(\left\{f_{1}, f_{2}, f_{3}\right\}\right)$, any triangle of $M / f_{n}$ that were to contain $f_{5}$ could contain at most one element from $\left\{f_{1}, f_{2}, f_{3}, f_{4}, d\right\}$. We know that $d \notin \mathrm{cl}_{M / f_{n}}\left(E\left(M / f_{n}\right)-\left\{f_{1}, f_{2}, f_{3}\right\}\right)$ as $d$ blocks the triad $\left\{f_{1}, f_{2}, f_{3}\right\}$ in $M / f_{n}$, and thus any triangle in this matroid containing $f_{5}$ must be disjoint from $\{d\}$. This, together with the fact which follows from 2.4.3 that $M \backslash d / f_{5}, f_{n}$ is 3-connected, implies that $M / f_{5}, f_{n}$ must be 3-connected.

2.4.4.4. The elements of $\left\{t_{1}, t_{2}, t_{3}\right\}$ are distinct. 
Proof. If $t_{i}=t_{j}$ for some distinct $i, j \in\{1,2,3\}$, then we see that $\left\{t_{i}, f_{i}, f_{j}\right\}$ is a triangle in $M / f_{n}$, contradicting the fact established above that $M \backslash d / f_{i}, f_{n}$ is 3-connected.

2.4.4.5. If $i \neq j$, then $t_{j} \in \operatorname{cl}_{M / f_{n} \backslash d}\left(\left\{f_{1}, f_{2}, f_{3}, t_{i}\right\}\right)$.

Proof. It is easily seen that, as $\left\{f_{i}, t_{i}, d\right\}$ and $\left\{f_{j}, t_{j}, d\right\}$ are triangles, $\left\{f_{i}, f_{j}, t_{i}, t_{j}\right\}$ is a circuit in $M / f_{n}$. But $\left\{f_{i}, f_{j}, t_{i}\right\} \subseteq\left\{f_{1}, f_{2}, f_{3}, t_{i}\right\}$ and the claim follows.

Let $l$ be the least integer such that $t_{i}=f_{l}$ for some $i \in\{1,2,3\}$. Then $\left\{t_{1}, t_{2}, t_{3}\right\}-\left\{t_{i}\right\} \subseteq \operatorname{cl}_{M / f_{n} \backslash d}\left(\left\{f_{1}, f_{2}, \ldots, f_{l}\right\}\right)-\left\{f_{1}, f_{2}, \ldots, f_{l}\right\}$ by 2.4.4.5. But if $l$ is even, then $\left\{f_{1}, f_{2}, \ldots, f_{l}\right\}$ is closed in $M / f_{n} \backslash d$, while if $l$ is odd, then $\operatorname{cl}_{M / f_{n} \backslash d}\left(\left\{f_{1}, f_{2}, \ldots, f_{l}\right\}\right)-\left\{f_{1}, f_{2}, \ldots, f_{l}\right\}=\left\{f_{l+1}\right\}$. The lemma follows from this contradiction.

Lemma 2.4.5. Let $M$ be a 3-connected matroid with $|E(M)|>7$ and let d be an element of $M$ such that $M \backslash d$ is 3-connected with $F=\left(f_{1}, f_{2}, f_{3}, f_{4}, f_{5}\right)$ a maximal flan of $M \backslash d$. Assume that $\mathrm{fcl}_{M}(F \cup\{d\})$ contains no triangles or triads. If $F$ is blocked by $d$, then $M$ has a detachable pair contained in $\mathrm{cl}_{M}(F \cup\{d\})$.

Proof. Assume that the lemma fails. Let $H=E(M)-(F \cup\{d\})$ and $G=$ $\operatorname{cl}_{M}(F \cup\{d\}) \cap H$. By Lemma 2.4.3, we have that $M \backslash d / f_{5}, f_{i}$ is 3-connected for all $i \in\{1,2,3\}$. As we are working under the assumption that $M$ does not contain a detachable pair, we must then have triangles $\left\{d, f_{1}, g_{1}\right\},\left\{d, f_{2}, g_{2}\right\}$ and $\left\{d, f_{3}, g_{3}\right\}$ in $M / f_{5}$, and, as $\operatorname{fcl}_{M}(F \cup\{d\})$ contains no triangles, appending $f_{5}$ to these three circuits now gives $\left\{d, f_{1}, g_{1}, f_{5}\right\},\left\{d, f_{2}, g_{2}, f_{5}\right\}$ and $\left\{d, f_{3}, g_{3}, f_{5}\right\}$ as 4 -element circuits in the matroid $M$. Note that if $g_{i} \in F$ for any $i \in\{1,2,3\}$, then $d$ does not block $F$. It follows routinely from this that $g_{1}, g_{2}$ and $g_{3}$ are distinct. We now proceed to show that there is another element of $M$ that we can delete in place of $d$ to obtain a related flan. 
2.4.5.1. There exists an element $z \in E(M)$ such that the following properties hold:

(i) $\left(d, f_{5}, f_{3}, g_{3}, z\right)$ is a maximal flan in $M \backslash f_{4}$, and

(ii) $z \in G-\left\{g_{1}, g_{2}, g_{3}\right\}$,

Proof. Consider the matroid $M \backslash f_{4}$. As the triad $\left\{f_{3}, f_{4}, f_{5}\right\}$ of $M \backslash d$ is blocked by $d$ in $M$, we have that $\left\{d, f_{5}, f_{3}\right\}$ is a triad in $M \backslash f_{4}$. As we have seen, $\left\{d, f_{5}, f_{3}, g_{3}\right\}$ is a circuit in $M$, and so is also a circuit in $M \backslash f_{4}$. So $g_{3}$ is contained in the closure of the 3 -separating set $\left\{d, f_{3}, f_{5}\right\}$ of $M \backslash d$ and therefore $\operatorname{si}\left(M \backslash f_{4} / g_{3}\right)$ is not 3-connected. By Bixby's Lemma then, as $\left\{f_{4}, g_{3}\right\}$ cannot be a detachable pair, $g_{3}$ must be contained in some triad $\Gamma$ of $M \backslash f_{4}$. By orthogonality, $\Gamma$ must meet the circuit $\left\{d, f_{5}, f_{3}, g_{3}\right\}$. It cannot be the case that $\Gamma \subseteq\left\{d, f_{5}, f_{3}, g_{3}\right\}$, as otherwise $\lambda_{M \backslash f_{4}}\left(\left\{d, f_{5}, f_{3}, g_{3}\right\}\right)=1$. So $\left|\Gamma \cap\left\{d, f_{5}, f_{3}\right\}\right|=1$. Recalling that $\left\{d, f_{1}, f_{5}, g_{1}\right\}$ and $\left\{d, f_{2}, f_{5}, g_{2}\right\}$ are circuits of $M \backslash f_{4}$, it now follows from orthogonality that $\Gamma \cap\left\{d, f_{5}, f_{3}, g_{3}\right\}=\left\{f_{3}, g_{3}\right\}$. Let $z$ be the remaining element of $\Gamma$. Circuit exchange on $\left\{d, f_{1}, g_{1}, f_{5}\right\}$ and $\left\{d, f_{2}, g_{2}, f_{5}\right\}$ gives a circuit of $M \backslash f_{4}$ contained in $\left\{d, f_{1}, g_{1}, f_{2}, g_{2}\right\}$. Orthogonality with $\Gamma$ now implies that $z \notin\left\{f_{1}, g_{1}, f_{2}, g_{2}\right\}$ and consequently that $z \notin F \cup\left\{g_{1}, g_{2}, g_{3}\right\}$. It is now clear that $\left(d, f_{5}, f_{3}, g_{3}, z\right)$ is a flan in $M \backslash f_{4}$. If this flan is not maximal, then $F \cup\{d\}$ contains a detachable pair by Lemma 2.4.4. This proves (i). By Lemma 2.4.3, $M \backslash f_{4} / f_{3}, z$ is 3-connected. As $M$ has no detachable pairs, $M$ must therefore contain a 4-element cocircuit $\left\{f_{4}, f_{3}, z, h_{1}\right\}$ for some $h_{1}$. We now show that $\sqcap\left(\left\{f_{3}, f_{4}\right\}, H\right)=0$. If $f_{4} \in \operatorname{cl}(H)$, then $\left\{f_{3}, f_{4}, f_{5}\right\}$ fails to be a cocircuit of $M \backslash d$. Similarly, as $\left\{f_{1}, f_{2}, f_{3}\right\}$ is a triad of $M \backslash d$, we also see that $f_{3} \notin \operatorname{cl}\left(H \cup\left\{f_{4}\right\}\right)$. Hence $\sqcap\left(\left\{f_{3}, f_{4}\right\}, H\right)=0$ as evisioned. Thus any circuit of $M$ containing $\left\{f_{3}, f_{4}\right\}$ must meet $\left\{f_{1}, f_{2}, f_{5}, d\right\}$. The circuit $\left\{f_{4}, f_{3}, z, h_{1}\right\}$ previously established is such a circuit. This shows that $h_{1} \in\left\{f_{5}, d, f_{2}, f_{1}\right\}$ and consequently that $z \in \operatorname{cl}_{M}(F \cup\{d\})$. This completes the proof of (ii).

We now work towards a proof that $M \backslash g_{1}, f_{2}$ must be 3-connected. 
2.4.5.2. Every 4-element subset of $G$ is a circuit of $M$.

Proof. Let $J$ be a 4-element subset of $G$. If $J$ is independent, then, as $\left\{f_{1}, f_{2}, f_{3}, d\right\}$ is a cocircuit, we have $r\left(J \cup\left\{f_{1}\right\}\right)=5$. Similarly, as $\left\{f_{3}, f_{4}, f_{5}, d\right\}$ is a cocircuit, we then have $r\left(J \cup\left\{f_{1}, f_{3}\right\}\right)=6$. This contradicts the fact that $r(\operatorname{cl}(F \cup\{d\}))=5$.

2.4.5.3. $\left\{f_{1}, f_{2}, g_{1}, g_{2}\right\}$ is coindependent in $M$.

Proof. We already know that each of $C_{1}=\left\{f_{1}, f_{2}, f_{3}, d\right\}, C_{2}=\left\{f_{3}, f_{4}, f_{5}, d\right\}$ and $C_{3}=\left\{f_{3}, g_{3}, z, f_{4}\right\}$ are cocircuits of $M$. Suppose that $C_{4}=\left\{f_{1}, f_{2}, g_{1}, g_{2}\right\}$ is also a cocircuit. Then $E(M)-\left(C_{1} \cup C_{2} \cup C_{3} \cup C_{4}\right)$ is a flat of rank at most $r(M)$-4. But by 2.4.5.1 (ii), $\left(C_{1} \cup C_{2} \cup C_{3} \cup C_{4}\right) \subseteq \operatorname{cl}(F \cup\{d\})$; which has rank 5. Thus $\left(C_{1} \cup C_{2} \cup C_{3} \cup C_{4}\right)=F \cup\{d\} \cup\left\{g_{1}, g_{2}, g_{3}, z\right\}$ is 2-separating. This implies the existence of a contradictory 2-separation in $M$ unless $|M|=11$ with $\{h\}=E(M)-\left(C_{1} \cup C_{2} \cup C_{3} \cup C_{4}\right)$. In the exceptional situation, it must be that $h \in G$ with $G=\left\{g_{1}, g_{2}, g_{3}, z, h\right\}$. By 2.4.5.2, $M$ now has a restriction isomorphic to $U_{3,5}$. As $M$ does not contain a detachable pair, this contradicts Lemma 2.2.5. Thus $\left\{f_{1}, f_{2}, g_{1}, g_{2}\right\}$ must be coindependent.

2.4.5.4. $M$ has no 4-element cocircuit containing $\left\{g_{1}, f_{2}\right\}$.

Proof. Suppose that $C$ is a cocircuit with $g_{1}, f_{2} \in C$. By 2.4.5.3, this cocircuit cannot be $\left\{f_{1}, f_{2}, g_{1}, g_{2}\right\}$. This; together with the fact that $\left\{f_{1}, g_{1}, d, f_{5}\right\}$ and $\left\{f_{2}, g_{2}, d, f_{5}\right\}$ are circuits of $M$, implies by orthogonality that $C \cap\left\{d, f_{5}\right\} \neq \emptyset$. The set $\left\{f_{1}, f_{2}, f_{3}, f_{4}\right\}$ is also a circuit of $M$ and so $C \cap\left\{f_{1}, f_{3}, f_{4}\right\} \neq \emptyset$ as well. By 2.4.5.2, $\left\{g_{1}, g_{2}, g_{3}, z\right\}$ is another circuit of $M$, and again, by orthogonality, we have $C \cap\left\{g_{2}, g_{3}, z\right\} \neq \emptyset$. But now $C$ is a 4 -element cocircuit with at least five distinct elements.

It follows from 2.4.5.4 that $M \backslash g_{1}, f_{2}$ contains no series pairs. Suppose then that $(P, Q)$ is a 2-separation in $M \backslash g_{1}, f_{2}$. The set $\left\{f_{1}, f_{3}, d\right\}$ is a triad of $M \backslash g_{1}, f_{2}$, and without loss of generality, $\left|P \cap\left\{f_{1}, f_{3}, d\right\}\right| \geq 2$. By 
Lemma 1.2.6, we may now assume that $P$ is fully closed. So $\left\{f_{1}, f_{3}, d\right\} \subseteq P$. If $f_{4} \in P$ also, then it follows that $f_{5} \in P$ as $f_{5} \in \mathrm{cl}_{M}^{*}\left(\left\{f_{3}, f_{4}, d\right\}\right)$. But then $P$ spans $\left((F \cup G \cup\{d\})-\left\{g_{1}, f_{2}\right\}\right)$ which implies that $P \cup\left\{g_{1}, f_{2}\right\}$ is 2-separating in $M$. Hence we are forced to have $f_{4} \in Q$ and, similarly, $f_{5} \in Q$. It must then be the case that $g_{3} \in Q$ also as $\left\{f_{3}, f_{5}, g_{3}, d\right\}$ is a circuit. Consider now the cocircuit $\left\{z, f_{3}, g_{3}, f_{4}\right\}$. Suppose that $z \in Q$. Then taking the full closure of $Q$ we see that $f_{3} \in \operatorname{fcl}(Q)$. But then $d \in \operatorname{fcl}(Q)$ also as $\left\{d, f_{3}, g_{3}, f_{5}\right\}$ is a circuit, and, consequently $f_{1} \in \operatorname{fcl}(Q)$ too. But now $\operatorname{fcl}(Q)$ spans $\left((F \cup G \cup\{d\})-\left\{g_{1}, f_{2}\right\}\right)$ and we have a 2-separation $\left(P-\operatorname{fcl}(Q), \operatorname{fcl}(Q) \cup\left\{g_{1}, f_{2}\right\}\right)$ in $M$. Therefore we are forced to have $z \in P$. Recalling from 2.4.5.1 that $\left(d, f_{5}, f_{3}, g_{3}, z\right)$ is a maximal flan of $M \backslash f_{4}$, observe that $f_{1} \notin \mathrm{cl}_{M \backslash g_{1}, f_{2}}\left(\left\{f_{3}, d, z\right\}\right)$. So $\left\{f_{1}, f_{3}, d, z\right\} \subseteq P$ is independent, while $r\left(\left\{f_{4}, f_{5}, g_{3}\right\}\right) \geq 3$. Let $R=\left\{f_{1}, f_{3}, f_{4}, f_{5}, g_{3}, d, z\right\}$. In the restriction $M \mid R$ we now have $r(P \cap R)+r(Q \cap R)-r(R) \geq 4+3-5=2$. That is to say, $(P \cap R)$ fails to be 2 -separating in $M \mid R$. This contradicts Lemma 1.2.5 and completes the proof of the lemma. 


\section{Chapter 3}

\section{Global Structure in a Minor}

Suppose that $M$ is a 3-connected matroid with an element $d$ such that $M \backslash d$ is 3-connected. Naturally, the approach we take in an attempt to find a detachable pair in $M$ is to study the minor $M \backslash d$. In some situations, the minor is particularly highly structured and in this chapter we deal with two potential general configurations that can arise.

\subsection{Sequential Minor}

In the event that $M \backslash d$ is sequential, we are guaranteed a detachable pair in $M$ as follows.

Lemma 3.1.1. Let $M$ be a 3 -connected matroid with $|E(M)|>9$ containing no triangles or triads, and with an element d such that $M \backslash d$ is 3-connected. If $M \backslash d$ is sequential, then $M$ contains a detachable pair.

Proof. Suppose that $\left(x_{1}, \ldots, x_{n}\right)$ is a sequential ordering of $E(M \backslash d)$. As $M$ contains no triangles, $\left\{x_{1}, x_{2}, x_{3}\right\}$ and $\left\{x_{n-2}, x_{n-1}, x_{n}\right\}$ must be triads of $M \backslash d$. Assume that $M$ has no detachable pair.

3.1.1.1. $M$ does not contain a rank-3 set having 5 elements. 
Proof. As $M$ does not contain any triangles, any such set must be isomorphic to $U_{3,5}$. This contradicts Lemma 2.2.5.

3.1.1.2. If $x_{i} \in\left\{x_{1}, x_{2}, x_{3}\right\}$ and $x_{j} \in\left\{x_{n-2}, x_{n-1}, x_{n}\right\}$, then $M \backslash d / x_{i}, x_{j}$ is 3-connected unless $x_{i}$ and $x_{j}$ are contained in a 4-element circuit $C$ with $\left|\left\{x_{1}, x_{2}, x_{3}\right\} \cap C\right|=2=\left|\left\{x_{n-2}, x_{n-1}, x_{n}\right\} \cap C\right|$.

Proof. By Lemma 1.2.21, $M \backslash d / x_{i}$ is 3 -connected for each $i \in\{1,2,3\}$. If there is no 4 -element circuit containing exactly two elements of $\left\{x_{1}, x_{2}, x_{3}\right\}$ and two elements of $\left\{x_{n-2}, x_{n-1}, x_{n}\right\}$, then no element of $\left\{x_{n-2}, x_{n-1}, x_{n}\right\}$ can be in a triangle of $M \backslash d / x_{i}$ for $i \in\{1,2,3\}$, and the result follows from another application of Lemma 1.2.21.

3.1.1.3. There exists at most one 4 -element circuit $C$ such that $\mid\left\{x_{1}, x_{2}, x_{3}\right\} \cap$ $C|=2=|\left\{x_{n-2}, x_{n-1}, x_{n}\right\} \cap C \mid$.

Proof. If there exists two distinct such circuits, then it follows that $\sqcap_{M \backslash d}\left(\left\{x_{1}, x_{2}, x_{3}\right\},\left\{x_{n-2}, x_{n-1}, x_{n}\right\}\right)=2$ so that $|E(M)| \leq 9$.

Note that if $x_{k}$ is a guts element in the sequential ordering $\left(x_{1}, \ldots, x_{n}\right)$ of $M \backslash d$, then by Bixby's Lemma, $\operatorname{co}\left(M \backslash d, x_{k}\right)$ is 3-connected. Therefore in order then for $\left\{d, x_{k}\right\}$ to fail to be a detachable pair, $x_{k}$ must be contained in a triad $\Gamma_{k}$ of $M \backslash d$ with $\Gamma_{k} \cap\left\{x_{1}, \ldots, x_{k-1}\right\} \neq \emptyset \neq \Gamma_{k} \cap\left\{x_{k+1}, \ldots, x_{n}\right\}$. As $d$ blocks each of these triads in $M, \Gamma_{k} \cup\{d\}$ is a 4-element cocircuit for each $k$. If $L$ is a 4-element coline of $M \backslash d$, then, again, as $d$ blocks every triad of $M \backslash d$, it must be that $M^{*} \mid(L \cup\{d\}) \cong U_{3,5}$. Therefore by Lemma 2.2.5, $M$ contains a detachable pair. So $x_{4}$ is certainly not within the coclosure of $\left\{x_{1}, x_{2}, x_{3}\right\}$. Thus $x_{4} \in \operatorname{cl}\left(\left\{x_{1}, x_{2}, x_{3}\right\}\right)$, and similarly, $x_{n-3} \in \operatorname{cl}\left(\left\{x_{n-2}, x_{n-1}, x_{n}\right\}\right)$. As the labelling of the elements of $\left\{x_{1}, x_{2}, x_{3}\right\}$ is arbitrary with respect to the sequential ordering of $M \backslash d$, we may assume that $x_{3} \in \Gamma_{4}$. Similarly, we may assume that $x_{n-2} \in \Gamma_{n-3}$.

3.1.1.4. $M \backslash d / x_{3}, x_{j}$ and $M \backslash d / x_{n-2}, x_{i}$ are 3 -connected for all $i \in\{1,2,3\}$, $j \in\{n-2, n-1, n\}$. 
Proof. As $x_{3} \in \Gamma_{4}$, it follows that $x_{3} \notin \mathrm{cl}_{M \backslash d}\left(\left\{x_{1}, x_{2}, x_{n-2}, x_{n-1}, x_{n}\right\}\right)$ and so by 3.1.1.2, $M \backslash d / x_{3}, x_{j}$ is 3-connected for $j \in\{n-2, n-1, n\}$. Similarly $M \backslash d / x_{n-2}, x_{i}$ is 3 -connected for $i \in\{1,2,3\}$.

3.1.1.5. $x_{4}$ and $x_{n-3}$ are the only guts elements in the sequential ordering $\left(x_{1}, \ldots, x_{n}\right)$ of $M \backslash d$.

Proof. Suppose that $\mathrm{cl}\left(\left\{x_{1}, x_{2}, x_{3}\right\}\right)-\left\{x_{1}, x_{2}, x_{3}, x_{4}\right\}$ is non-empty; containing an element $x_{5}$. Now $x_{3} \in \operatorname{cl}^{*}\left(E(M \backslash d)-\left\{x_{1}, x_{2}, x_{3}, x_{5}\right\}\right)$ so that $x_{3} \notin \mathrm{cl}\left(\left\{x_{1}, x_{2}, x_{5}\right\}\right)$. But this implies that $\left\{x_{1}, x_{2}, x_{5}\right\}$ is a contradictory triangle in $M \backslash d$. Thus $\operatorname{cl}\left(\left\{x_{1}, x_{2}, x_{3}\right\}\right)=\left\{x_{1}, x_{2}, x_{3}, x_{4}\right\}$. Similarly, $\operatorname{cl}\left(\left\{x_{n}, x_{n-1}, x_{n-2}\right\}\right)=\left\{x_{n}, x_{n-1}, x_{n-2}, x_{n-3}\right\}$. Suppose now that there exists some other guts element $x_{p}$ in the sequential ordering. Then $\Gamma_{p}$ must be disjoint from both $\left\{x_{1}, x_{2}, x_{3}\right\}$ and $\left\{x_{n}, x_{n-1}, x_{n-2}\right\}$. If $M$ does not contain a detachable pair, there must thus exist 4-element circuits $\left\{d, x_{3}, x_{n-1}, \alpha\right\}$ and $\left\{d, x_{3}, x_{n}, \beta\right\}$ in $M$. Now both $\Gamma_{n-3} \cup\{d\}$ and $\Gamma_{p} \cup\{d\}$ are cocircuits of $M$. By orthogonality, it must be that $\alpha, \beta \in \Gamma_{n-3} \cup\{d\}$ and $\alpha, \beta \in \Gamma_{p} \cup\{d\}$, and consequently that $\alpha=\beta$. But now it follows that $\left\{x_{3}, d, \alpha, x_{n-1}, x_{n}\right\}$ is a rank-3 set of $M$ with 5 elements. This is a contradiction to 3.1.1.1 and as such, the element $x_{p}$ cannot exist.

3.1.1.6. $\left|\mathrm{cl}_{M \backslash d}^{*}\left(\left\{x_{1}, x_{2}, x_{3}, x_{4}\right\}\right)-\left\{x_{1}, x_{2}, x_{3}, x_{4}\right\}\right|=1$.

Proof. Suppose otherwise, letting

$$
G^{*}=\operatorname{cl}_{M \backslash d}^{*}\left(\left\{x_{1}, x_{2}, x_{3}, x_{4}\right\}\right)-\left\{x_{1}, x_{2}, x_{3}, x_{4}\right\} .
$$

We know that $G^{*} \neq \emptyset$ because, as we have seen

$$
\sqcap\left(\left\{x_{1}, x_{2}, x_{3}\right\},\left\{x_{n}, x_{n-1}, x_{n-2}\right\}\right) \neq 2 .
$$

So $G^{*} \geq 2$. Note that by 3.1 .1 .5 , we also have the equality

$$
G^{*}=\mathrm{cl}_{M \backslash d}^{*}\left(\left\{x_{n}, x_{n-1}, x_{n-2}, x_{n-3}\right\}\right)-\left\{x_{n}, x_{n-1}, x_{n-2}, x_{n-3}\right\} .
$$


Now $r(M \backslash d)=4+\left|G^{*}\right| \geq 6$. It follows from this that

$$
r_{M \backslash d}\left(\left\{x_{1}, x_{2}, x_{3}, x_{n}, x_{n-1}, x_{n-2}\right\}\right)=6 .
$$

Equivalently, $\sqcap\left(\left\{x_{1}, x_{2}, x_{3}\right\},\left\{x_{n}, x_{n-1}, x_{n-2}\right\}\right)=0$. Now, by 3.1.1.2, $M \backslash d / x_{i}, x_{j}$ is 3-connected for all $x_{i} \in\left\{x_{1}, x_{2}, x_{3}\right\}, x_{j} \in\left\{x_{n}, x_{n-1}, x_{n-2}\right\}$. Consider the circuit $C_{1}=\left\{x_{1}, x_{n}, d, \alpha\right\}$ of $M$. By orthogonality, it must be that $\alpha \in \Gamma_{4} \cap \Gamma_{n-3}$. Similarly, $C_{2}=\left\{x_{2}, x_{n}, d, \beta\right\}$ is a circuit of $M$ and by orthogonality, $\beta \in \Gamma_{4} \cap \Gamma_{n-3}$. It follows that $\alpha=\beta$. But this contradicts 3.1.1.1.

The only remaining possibility is that $E(M \backslash d)=\left\{x_{1}, \ldots, x_{9}\right\}$. Here $\Gamma_{4}=\left\{x_{3}, x_{4}, x_{5}\right\}$ and $\Gamma_{6}=\left\{x_{5}, x_{6}, x_{7}\right\}$ are triads of $M \backslash d$ and consequently, as $r(M \backslash d)=5,\left\{x_{1}, x_{2}, x_{8}, x_{9}\right\}$ must be a circuit. Consider the circuits $\left\{x_{3}, d, x_{9}, \alpha\right\}$ and $\left\{x_{3}, d, x_{8}, \beta\right\}$ of $M$. It follows from 3.1.1.1 that $\alpha \neq \beta$, and we know from orthogonality that $\alpha, \beta \in \Gamma_{6}$. Thus $\{\alpha, \beta\} \cap\left\{x_{6}, x_{7}\right\} \neq$ $\emptyset$. Therefore, in the matroid $M / x_{3}, d$ is contained in a triangle $T$ with $T \cap\left\{x_{6}, x_{7}\right\} \neq \emptyset \neq T \cap\left\{x_{8}, x_{9}\right\}$. Assume for the moment that $x_{8} \in T$. In $M / x_{3}$, the element $d$ now lies within $\operatorname{cl}_{M / x_{3}}\left(\left\{x_{7}, x_{8}, x_{9}\right\}\right)$. Thus $d$ is also contained in a second triangle $T^{\prime}$ with $x_{9} \in T$ and $T \cap\left\{x_{6}, x_{7}\right\} \neq \emptyset$. As $M / x_{3}$ cannot contain a 4 -point line, it must be that $T \cap T^{\prime}=\{d\}$. We have symmetry between $x_{8}$ and $x_{9}$, and so we may assume then that $\left\{x_{8}, d, x_{6}\right\}$ and $\left\{x_{9}, d, x_{7}\right\}$ are the aforementioned triangles in $M / x_{3}$. These triangles then extend to circuits $\left\{x_{3}, d, x_{6}, x_{8}\right\}$ and $\left\{x_{3}, d, x_{7}, x_{9}\right\}$ in $M$. Now examining the matroid $M / x_{7}$ and repeating these arguments, we find that there must exist circuits $\left\{x_{7}, d, x_{1}, x_{m}\right\}$ and $\left\{x_{7}, d, x_{2}, x_{n}\right\}$ where $\left\{x_{m}, x_{n}\right\}=\left\{x_{3}, x_{4}\right\}$. But now, regardless of whether $x_{m}=x_{3}$ or $x_{m}=x_{4}$, we now have a five-element set with rank three. The result now follows from this final contradiction of 3.1.1.1.

In the next two lemmas we use the above result on sequential minors to extend our results from section 2.4 
Lemma 3.1.2. Let $M$ be a 3-connected matroid where $|E(M)| \geq 9$ with an element $d$ such that $M \backslash d$ is 3-connected. Let $X$ be a sequential 3-separating set of $M \backslash d$. Assume that $\mathrm{fcl}_{M}(X \cup\{d\})$ contains no triangles or triads. Then either

(i) $X$ is a fully closed triad of $M \backslash d$ and $X \cup\{d\}$ is a cocircuit in $M$, or

(ii) $\mathrm{fcl}_{M \backslash d}(X)$ is a 5-element set that can be ordered as a maximal flan in $M \backslash d$ where $d \in \operatorname{cl}_{M}(X)$ and $\mathrm{fcl}_{M \backslash d}(X) \cup\{d\}$ is fully closed in $M$, or

(iii) $\mathrm{fcl}_{M}(X \cup\{d\})$ contains a detachable pair.

Proof. We shall assume that (iii) does not hold and show that this forces (i) or (ii) to apply. By Lemma 3.1.1, we may assume that $M \backslash d$ is not sequential. Let $\left(f_{1}, \ldots, f_{n}\right)$ be a sequential ordering of $\mathrm{fcl}_{M \backslash d}(X)$ where $\left\{f_{1}, f_{2}, f_{3}\right\}$ is a triad in $M \backslash d$. If $n=3$, then (i) holds. So assume that this is not the case. If $f_{4} \in \operatorname{cl}_{M \backslash d}^{*}\left(\left\{f_{1}, f_{2}, f_{3}\right\}\right)$, then $\left\{f_{1}, f_{2}, f_{3}, f_{4}\right\}$ is a coseqment of $M \backslash d$. As $d$ must block each triad of this cosegment in $M$, we find ourselves in a situation in which $M^{*}$ has a minor isomorphic to $U_{3,5}$. This contradicts Lemma 2.2.5. Therefore $f_{4} \in \operatorname{cl}_{M \backslash d}\left(\left\{f_{1}, f_{2}, f_{3}\right\}\right)$. By Bixby's Lemma, $\operatorname{co}\left(M \backslash d, f_{4}\right)$ is 3 -connected, and so $f_{4}$ must be contained in a triad $\Gamma$ which meets both $\left\{f_{1}, f_{2}, f_{3}\right\}$ and $E(M \backslash d)-\left\{f_{1}, f_{2}, f_{3}, f_{4}\right\}$. Without loss of generality, we may assume that $\Gamma=\left\{f_{3}, f_{4}, f_{5}\right\}$. We now have a 5element flan in $M \backslash d$. It follows from Lemma 2.4.4 that this flan must be maximal. Suppose that $\left\{f_{1}, f_{2}, f_{3}, f_{4}, f_{5}\right\}$ fails to be fully closed in $M \backslash d$. Then $f_{6} \in \mathrm{cl}_{M \backslash d}^{*}\left(\left\{f_{1}, f_{2}, f_{3}, f_{4}, f_{5}\right\}\right)=\mathrm{cl}_{M \backslash d}^{*}\left(\left\{f_{1}, f_{2}, f_{3}, f_{4}\right\}\right)$. Note also that if $f_{n} \in \mathrm{cl}_{M \backslash d}\left(\left\{f_{1}, \ldots, f_{n-1}\right\}\right)$, then $\operatorname{co}\left(M \backslash d, f_{n}\right)$ is 3 -connected and so either $M$ contains a detachable pair, or $f_{n}$ is contained in a triad $\Gamma_{n}$ of $M \backslash d$ where $\left(\operatorname{fcl}_{M \backslash d}(X)-\left\{f_{n}\right\}\right) \cap \Gamma_{n} \neq \emptyset \neq \Gamma_{n} \cap\left(E(M \backslash d)-\mathrm{fcl}_{M \backslash d}(X)\right)$. The second possibility contradicts the fact that $\left(f_{1}, \ldots, f_{n}\right)$ is fully closed. So $f_{n} \in \mathrm{cl}_{M \backslash d}^{*}\left(\left\{f_{1}, \ldots, f_{n-1}\right\}\right)$. By repeated use of Lemma 1.2.10, together with the fact that $E(M \backslash d)-\mathrm{fcl}_{M \backslash d}(X)$ cannot be independent in $M \backslash d$, we deduce 
that $\left(\mathrm{fcl}_{M \backslash d}(X)-\left\{f_{n}\right\},\left\{f_{n}\right\}, E(M \backslash d)-\mathrm{fcl}_{M \backslash d}(X)\right)$ is a cyclic 3-separation of $M \backslash d$. By Bixby's Lemma then, we know that $M \backslash d / f_{n}$ must be 3-connected.

3.1.2.1. $M \backslash d / f_{n}, f_{k}$ is 3-connected for each $k \in\{5,1,2,3\}$.

Proof. Certainly, $\left(\left\{f_{1}, f_{2}, f_{3}, f_{4}\right\},\left\{f_{5}\right\}, E(M \backslash d)-\left\{f_{1}, f_{2}, f_{3}, f_{4}, f_{5}, f_{n}\right\}\right)$ is a cyclic 3-separation of $M \backslash d / f_{n}$. Hence $\operatorname{si}\left(M \backslash d / f_{n}, f_{5}\right)$ is 3-connected and consequently, $M \backslash d / f_{n}, f_{5}$ is 3-connected unless there exists a 4-element circuit $C$ of $M \backslash d$ with $\left\{f_{5}, f_{n}\right\} \subseteq C$. By orthogonality, the circuit $C$ must contain at least two elements from the triad $\left\{f_{3}, f_{4}, f_{5}\right\}$. The remaining element of $C$ may or may not be contained in $E(M \backslash d)-\mathrm{fcl}_{M \backslash d}(X)$. In the first instance, $\operatorname{fcl}_{M \backslash d}(X)$ fails to be fully closed, while in the second we contradict the fact that $f_{n} \in \mathrm{cl}_{M \backslash d}^{*}\left(E(M \backslash d)-\mathrm{fcl}_{M \backslash d}(X)\right)$. Thus $M \backslash d / f_{n}, f_{5}$ must be 3-connected. For the remaining cases, we know from Lemma 1.2.21 that $\operatorname{si}\left(M \backslash d / f_{n}, f_{i}\right)$ is 3 -connected for each $i \in\{1,2,3\}$. The proof that $M \backslash d / f_{n}, f_{i}$ contains no parallel pairs for $i \in\{1,2,3\}$ now proceeds similarly to the $M \backslash d / f_{n}, f_{5}$ case.

With 3.1.2.1 in hand, we are left to deduce that $d$ must be together in a triangle with each element from $\left\{f_{5}, f_{1}, f_{2}, f_{3}\right\}$ in $M / f_{n}$. Using orthogonality with the two cocircuits $\left\{f_{1}, f_{2}, f_{3}, d\right\}$ and $\left\{f_{3}, f_{4}, f_{5}, d\right\}$, a straightforward check of the possible configurations shows that this is not feasible. Thus we conclude that $\left\{f_{1}, f_{2}, f_{3}, f_{4}, f_{5}\right\}=\operatorname{fcl}_{M \backslash d}(X)$. From Lemma 2.4.5, we know that $d \in \operatorname{cl}_{M}\left(\left\{f_{1}, f_{2}, f_{3}, f_{4}, f_{5}\right\}\right)$, and finally from Lemma 1.2.7 that $\mathrm{fcl}_{M \backslash d}(X) \cup\{d\}$ is fully closed in $M$. Part (ii) is satisfied and the proof of the lemma is complete.

The next result refines the outcome from the previous result in the case where our sequential 3-separating set is more substantial than a single triad.

Lemma 3.1.3. Let $M$ be a 3-connected matroid with $|E(M)| \geq 9$ and let $d$ be an element of $M$ such that $M \backslash d$ is 3-connected. Let $X$ be a sequential 3-separating set of $M \backslash d$ with $|X| \geq 4$. Assume that $\mathrm{fcl}_{M}(X \cup\{d\})$ contains no triangles or triads. Then either 
(i) $\operatorname{fcl}(X \cup\{d\})$ contains a detachable pair, or

(ii) $\operatorname{fcl}_{M \backslash d}(X) \cup\{d\}$ is a fully closed twisted 3-separator of $M$, or

(iii) $\operatorname{fcl}_{M \backslash d}(X) \cup\{d\}$ is a fully closed partial spider-like 3-separator of $M$.

Proof. We shall assume that (i) fails. By Lemma 3.1.2 then, $\mathrm{fcl}_{M \backslash d}(X)$ can be ordered as a 5-element flan which is maximal in $M \backslash d$ with $d \in \operatorname{cl}_{M}(X)$ and where $\operatorname{fcl}_{M \backslash d}(X) \cup\{d\}$ is fully closed in $M$. Let $F=\left(f_{1}, \ldots, f_{5}\right)$ be the natural labelling of the elements of the flan $\mathrm{fcl}_{M \backslash d}(X)$. We certainly have cocircuits $\left\{f_{1}, f_{2}, f_{3}, d\right\}$ and $\left\{f_{3}, f_{4}, f_{5}, d\right\}$ in $M$ as $d$ must block any triad of $M \backslash d$. From Lemma 2.4.3, we deduce the existence of 4-element circuits $\left\{f_{1}, f_{5}, d, \alpha\right\},\left\{f_{2}, f_{5}, d, \beta\right\}$ and $\left\{f_{3}, f_{5}, d, \gamma\right\}$ in $M$, where $\{\alpha, \beta, \gamma\} \subseteq F$, along with the circuit $\left\{f_{1}, f_{2}, f_{3}, f_{4}\right\}$.

3.1.3.1. If $C_{1} \subseteq F \cup\{d\}$ and $C_{2} \subseteq F \cup\{d\}$ are distinct 4-element cocircuits of $M$, then $F \cup\{d\} \subseteq C_{1} \cup C_{2}$. Similarly, if $C_{1} \subseteq F \cup\{d\}$ and $C_{2} \subseteq F \cup\{d\}$ are distinct 4-element circuits of $M$, then $F \cup\{d\} \subseteq C_{1} \cup C_{2}$.

Proof. The set $F \cup\{d\}$ is exactly 3-separating in $M$ with $r_{M}(F \cup\{d\})=$ $r_{M}^{*}(F \cup\{d\})=4$ and so $r(E(M)-(F \cup\{d\}))=r(M)-2$. Suppose that $C_{1} \subseteq(F \cup\{d\})$ and $C_{2} \subseteq(F \cup\{d\})$ are distinct 4-element cocircuits of $M$. Then $E(M)-\left(C_{1} \cup C_{2}\right)$ must be a flat of rank $r(M)-2$. Thus, if $x \in\left((F \cup\{d\})-\left(C_{1} \cup C_{2}\right)\right)$, it follows that $x \in \operatorname{cl}_{M}(E(M)-(F \cup\{d\}))$. But this contradicts the fact that every element of $F \cup\{d\}$ is contained in a some cocircuit which is itself contained in $F \cup\{d\}$. The proof of the dual result follows in the same manner due to the fact that $r_{M}(F \cup\{d\})=r_{M}^{*}(F \cup\{d\})=$ 4.

3.1.3.2. $M / f_{1} \backslash f_{2}, f_{5}$ is 3-connected.

Proof. Consider the 3 -connected matroid $M / f_{1}$. The set $\left\{f_{3}, f_{4}, f_{5}, d\right\}$ is a rank-3 cocircuit in this matroid, and so by Lemma $1.2 .14, \operatorname{co}\left(M / f_{1} \backslash f_{5}\right)$, and indeed $M / f_{1} \backslash f_{5}$ must be 3 -connected. Now by Bixby's Lemma, $M / f_{1} \backslash f_{2}, f_{5}$ 
is 3-connected unless $f_{2}$ is contained in a triad of $M / f_{1} \backslash f_{5}$ which meets both $\left\{f_{3}, f_{4}, d\right\}$ and $E\left(M / f_{1} \backslash f_{5}\right)-(F \cup\{d\})$. But as $F \cup\{d\}$ is fully closed in $M$, it follows from Lemma 1.2 .8 that $\left\{f_{2}, f_{3}, f_{4}, d\right\}$ is fully closed in $M / f_{1} \backslash f_{5}$. Thus $M / f_{1} \backslash f_{2}, f_{5}$ must be 3 -connected as required.

From 3.1.3.2, we now deduce the existence of a 4-element cocircuit $\left\{f_{1}, f_{2}, f_{5}, z\right\}$ where; as $F \cup\{d\}$ is fully closed in $M, z \in\left\{f_{3}, f_{4}\right\}$. With our previous knowledge that $\left\{f_{1}, f_{2}, f_{3}, d\right\}$ and $\left\{f_{3}, f_{4}, f_{5}, d\right\}$ are cocircuits in $M$, we can now conclude with the use of 3.1.3.1 that $\left\{f_{1}, f_{2}, f_{4}, f_{5}\right\}$ must be our cocircuit in question. All that remains is to examine the potential configurations of our 4-element circuits $\left\{f_{1}, f_{5}, d, \alpha\right\},\left\{f_{2}, f_{5}, d, \beta\right\}$ and $\left\{f_{3}, f_{5}, d, \gamma\right\}$; each of which is contained in $F \cup\{d\}$. If $\alpha=f_{3}$, we are forced by 3.1.3.1 to have $\gamma=f_{3}$ and $\beta=f_{4}$. In such a situation, it is easily checked against the definition by relabelling the ordered sequence $\left(f_{1}, f_{2}, f_{3}, f_{4}, f_{5}, d\right)$ as $\left(r_{1}, r_{2}, s_{2}, t_{1}, t_{2}, s_{1}\right)$ that $F \cup\{d\}$ is a twisted 3 -separator of $M$ so that (ii) holds. Similarly, if $\alpha=f_{4}$, we obtain a twisted 3 -separator in the same manner; which can be readily checked by setting $\left(f_{1}, f_{2}, f_{3}, f_{4}, f_{5}, d\right)$ as $\left(s_{1}, s_{2}, r_{1}, t_{2}, t_{1}, r_{2}\right)$. The final possibility arises when $\alpha=f_{2}$. In this case, 3.1.3.1 forces $\beta=f_{1}$ and $\gamma=f_{4}$. It is clear that $F \cup\{d\}$ is now a partial spider-like 3 -separator with associated partition $\left\{\left\{f_{3}, f_{4}, f_{5}, d\right\},\left\{f_{1}, f_{2}\right\}\right\}$ giving (iii). The combinatorics is complete and we conclude the proof of the lemma.

\subsection{Internally 4-Connected Minor}

Whenever the matroid $M \backslash d$ is internally 4-connected and has more than ten elements, we can also deduce the existence of a detachable pair in $M$, as is shown in the following.

Lemma 3.2.1. Let $M$ be a 3-connected matroid with $|E(M)|>10$. If $M$ contains no triangles or triads and $d \in E(M)$ is such that $M \backslash d$ is internally 4-connected, then $M$ contains a detachable pair. 
Proof. Assume that $M$ does not contain a detachable pair.

3.2.1.1. Every element of $M \backslash d$ is in a triad.

Proof. If $x \in E(M \backslash d)$ is not in a triad, then as $\operatorname{co}(M \backslash d, x)$ is not 3-connected, there must exist a cyclic 3-separation $(R,\{x\}, S)$ of $M \backslash d$. As $M \backslash d$ is internally 4-connected, at least one of $R$ and $S$ is a triad. But both $R$ and $S$ must by definition contain a circuit. Therefore we contradict the fact that $M \backslash d$ is 3-connected.

3.2.1.2. $M \backslash d$ contains at least four distinct triads.

Proof. This follows immediately from 3.2.1.1 and our assumption that $|E(M \backslash d)| \geq 10$.

3.2.1.3. For all $x \in E(M \backslash d)$, the matroid $M \backslash d / x$ is 3-connected.

Proof. If $M \backslash d / x$ fails to be 3 -connected for some $x \in E(M \backslash d)$, then by 1.2.11, there exists a vertical 3-separation $(P,\{x\}, Q)$ of $M \backslash d$. But then it is clear that both $P$ and $Q$ must be triads for $M \backslash d$ is internally 4-connected. This contradicts our assumption that $|E(M)|>10$.

3.2.1.4. If $S$ and $T$ are disjoint triads of $M \backslash d$, then for all $s \in S$, there exists some $t \in T$ such that $M \backslash d / s, t$ is 3-connected.

Proof. If $T$ is closed in $M \backslash d / s$, then the result follows immediately from Tutte's Triangle Lemma. So assume that this is not the case. Note that if $z \in\left(\mathrm{cl}_{M \backslash d / s}(T)-T\right)$, it follows that $z \in S$, for otherwise $T \cup\{z\}$ would be exactly 3-separating in $M \backslash d$. Suppose that $s_{1}, s_{2}$ are distinct elements of $\mathrm{cl}_{M \backslash d / s}(T)-T$. Then $\lambda_{M \backslash d / s}\left(T \cup\left\{s_{1}, s_{2}\right\}\right)=2$, implying that $\lambda_{M \backslash d}(T \cup S)=2$. But, as $|E(M)|>10$, this now contradicts the fact that $M \backslash d$ is internally 4-connected. Thus we may assume that $\left|\mathrm{cl}_{M \backslash d / s}(T)\right|=4$. There must now exist some $t \in T$ which is not contained in any triangle of $M \backslash d / s$. Applying Lemma 1.2.21 we see that $M \backslash d / s, t$ is 3-connected as required. 
3.2.1.5. If $S$ and $T$ are distinct triads of $M \backslash d$, then $|S \cap T| \in\{0,1\}$.

Proof. If $|S \cap T|=2$, then $\lambda_{M \backslash d}(S \cup T)=2$, which, as $|E(M)|>10$, again contradicts the fact that $M \backslash d$ is internally 4-connected.

3.2.1.6. Let $S=\left\{s_{1}, s_{2}, \alpha\right\}$ and $T=\left\{t_{1}, t_{2}, \alpha\right\}$ be intersecting triads of $M \backslash d$. Then for each $i \in\{1,2\}$, there exists $j \in\{1,2\}$ such that $M \backslash d / s_{i}, t_{j}$ is 3-connected.

Proof. Consider $M \backslash d / s_{1}$. If $T$ is closed in this matroid, the result follows from Tutte's Triangle Lemma. Otherwise, $s_{2} \in \mathrm{cl}_{M \backslash d / s_{1}}(T)$. But then, as $s_{1} \in$ $\mathrm{cl}_{M \backslash d}^{*}\left(T \cup\left\{s_{2}\right\}\right)$, we get that $\lambda_{M \backslash d}(S \cup T)=2$, which is a contradiction.

3.2.1.7. No element of $M \backslash d$ is contained in four distinct triads.

Proof. Suppose to begin with that $\alpha$ is an element which is contained in four distinct triads $\left\{r_{1}, r_{2}, \alpha\right\},\left\{s_{1}, s_{2}, \alpha\right\},\left\{t_{1}, t_{2}, \alpha\right\}$ and $\left\{u_{1}, u_{2}, \alpha\right\}$ of $M \backslash d$. By 3.2.1.6, $M$ must have a 4-element circuit which contains $\left\{r_{1}, s_{1}, d\right\}$, and by orthogonality with the cocircuits $\left\{t_{1}, t_{2}, d, \alpha\right\}$ and $\left\{u_{1}, u_{2}, d, \alpha\right\}$ of $M$, it is clear that this circuit must be $\left\{r_{1}, s_{1}, d, \alpha\right\}$. Similarly, $M$ must have some 4-element circuit which contains $\left\{r_{1}, t_{1}, d\right\}$, and this circuit must be $\left\{r_{1}, t_{1}, d, \alpha\right\}$. But now $M$ has a minor isomorphic to $U_{3,5}$, which contradicts Lemma 2.2.5.

3.2.1.8. $M \backslash d$ does not contain three pairwise disjoint triads.

Proof. Suppose that $\Gamma_{1}, \Gamma_{2}$ and $\Gamma_{3}$ are pairwise disjoint triads. As $E(M \backslash d)$ has at least ten elements, there must exist some $\alpha$ not contained in any of these triads. This $\alpha$ must be in some triad $\Gamma_{\alpha}$ of $M \backslash d$. Without loss of generality, we may assume that $\Gamma_{\alpha} \cap \Gamma_{1}=\emptyset$. Now by 3.2.1.4, there must exist some 4-element circuit $C$ in $M$ which contains $d, \alpha$ and at least one point from $\Gamma_{1}$. But now at least one of $\Gamma_{2} \cup\{d\}$ and $\Gamma_{3} \cup\{d\}$ is a 4-element cocircuit of $M$ whose intersection with the circuit $C$ is $\{d\}$. This is a contradiction by orthogonality. 
3.2.1.9. Each element of $M \backslash d$ is contained in at most two distinct triads.

Proof. By 3.2.1.7 above, we know that each element of $M \backslash d$ is contained in at most three distinct triads. Suppose that $\alpha$ is indeed contained in three distinct triads $\Gamma_{r}=\left\{r_{1}, r_{2}, \alpha\right\}, \Gamma_{s}=\left\{s_{1}, s_{2}, \alpha\right\}$ and $\Gamma_{t}=\left\{t_{1}, t_{2}, \alpha\right\}$. Suppose that $\Gamma$ is some triad of $M \backslash d$ which is distinct from each of $\Gamma_{r}, \Gamma_{s}$ and $\Gamma_{t}$. If $\left|\Gamma \cap\left(\Gamma_{r} \cup \Gamma_{s} \cup \Gamma_{t}\right)\right| \in\{0,1\}$, then we may assume up to an arbitrary choice of labelling that $\Gamma \cap\left\{r_{1}, s_{1}, s_{2}\right\}=\emptyset$. By 3.2.1.6, there must exist a 4-element circuit $C$ which contains $r_{1}, d$ and at least one element from $\left\{s_{1}, s_{2}\right\}$. Now as $C$ cannot contain both an element from both $\Gamma$ and $\Gamma_{t}$, we inevitably arrive at a contradiction by orthogonality in the matroid $M$.

Thus any triad of $M \backslash d$ other than $\Gamma_{r}, \Gamma_{s}$ and $\Gamma_{t}$ must intersect $\Gamma_{r} \cup \Gamma_{s} \cup \Gamma_{t}$ at exactly two points. As $|E(M)|>10$, there exists at least three other points $\delta, \mu$ and $\zeta$; none of which is contained in $\Gamma_{r} \cup \Gamma_{s} \cup \Gamma_{t}$, and each of which is contained in such a corresponding triad $\Gamma_{\delta}, \Gamma_{\mu}$ and $\Gamma_{\zeta}$. Supppose now that $\alpha$ is the only element of $M \backslash d$ which is contained in three triads. We know from 3.2.1.7 that $\alpha$ is contained in exactly three triads, and a quick check reveals that the only potentially allowable configuration that remains is where each element from $\Gamma_{r} \cup \Gamma_{s} \cup \Gamma_{t}$ apart from $\alpha$ is contained in exactly two triads of $M \backslash d$. However in this situation the three triads $\Gamma_{\delta}, \Gamma_{\mu}$ and $\Gamma_{\zeta}$ are pairwise disjoint and this contradicts 3.2.1.8.

Therefore some element of $\left\{r_{1}, r_{2}, s_{1}, s_{2}, t_{1}, t_{2}\right\}$ must also be contained in exactly three triads of $M \backslash d$. Our labelling maintains arbitrarity at this point and so we may assume without loss of generality that $s_{1}$ is such an element. Up to symmetry, there are now two possible situations to examine. Either $\left\{s_{1}, r_{1}, \delta\right\}$ and $\left\{s_{1}, r_{2}, \mu\right\}$ are triads, or $\left\{s_{1}, r_{1}, \delta\right\}$ and $\left\{s_{1}, t_{1}, \mu\right\}$ are triads. In the first instance, we know from 3.2.1.6 that either $\left\{s_{2}, r_{1}, d\right\}$ or $\left\{s_{2}, r_{2}, d\right\}$ is contained in a 4 -element circuit $C$ of $M$, but then at least one of $\Gamma_{\delta}, \Gamma_{\mu}$ and $\Gamma_{t}$ would be disjoint from $C$, which is a contradiction by orthogonality. So we may assume that we are in the second instance where $\left\{s_{1}, r_{1}, \delta\right\}$ and $\left\{s_{1}, t_{1}, \mu\right\}$ are triads. Recalling once more from 3.2.1.8 that no three triads 
may be pairwise disjoint, we note that there are two possibilities for the location of $\Gamma_{\zeta}$. Either $\Gamma_{\zeta}=\left\{r_{1}, t_{1}, \zeta\right\}$, or $\Gamma_{\zeta}=\left\{r_{2}, t_{2}, \zeta\right\}$. We shall use this fact shortly.

Consider the matroid $M \backslash d / s_{2}, \zeta$. If this matroid is 3-connected, then $M$ must have a 4 -element circuit which contains $\left\{d, s_{2}, \zeta\right\}$. But this contradicts orthogonality as whatever $\Gamma_{\zeta}$ may be, there always exists a triad of $M \backslash d$ which is disjoint from $C$. Hence $M \backslash d / s_{2}, \zeta$ must have some 2-separation $(P, Q)$. A quick check of orthogonality against the triads of $M \backslash d$ reveals that $M \backslash d$ cannot contain any 4-element circuits that contain $s_{2}$ and $\zeta$ and so $M \backslash d / s_{2}, \zeta$ cannot contain any parallel pairs, and we may freely apply Lemma 1.2.6 to this 2-separation. Therefore we may assume that $\Gamma_{t} \subseteq P$. This forces $s_{1} \in Q$, as otherwise $\left(P \cup\left\{s_{2}\right\}, Q\right)$ would be a 2-separation in $M \backslash d / \zeta$ thereby contradicting 3.2.1.3. Now if $\left\{r_{1}, r_{2}\right\} \subseteq Q$, then we may relabel to another 2-separation in which $\alpha \in Q, s_{1} \in Q$. But then $(P, Q \cup$ $\left\{s_{2}\right\}$ ) would be a contradictory 2-separation in $M \backslash d / \zeta$. So at least one element from $\left\{r_{1}, r_{2}\right\}$ must be in $P$, and, taking the coclosure of $P$, we may assume that $\left\{r_{1}, r_{2}\right\} \subseteq P$. So we have a 2 -separation $(P, Q)$ in which $\left\{r_{1}, r_{2}, t_{1}, t_{2}\right\} \subseteq P$. But recall that either $\left\{r_{1}, t_{1}, \zeta\right\}$ or $\left\{r_{2}, t_{2}, \zeta\right\}$ is a triad of $M \backslash d / s_{2}$. Thus $(P \cup\{\zeta\}, Q)$ is a 2 -separation in the 3 -connected matroid $M \backslash d / s_{2}$. The result follows from this final contradiction.

To clean up the remainder of the analysis in this section we shall make the following definition. A trident of a matroid is a five-element set which consists of two triads meeting at a point. This terminology is convenient for our immediate purposes and its use is restricted to this section.

3.2.1.10. Let $\Psi_{1}$ and $\Psi_{2}$ be two tridents of $M \backslash d$. Then $\Psi_{1} \cap \Psi_{2} \neq \emptyset$.

Proof. Assume otherwise. Suppose that there exists some $x \in(E(M \backslash d)-$ $\left.\left(\Psi_{1} \cup \Psi_{2}\right)\right)$. By 3.2.1.1, $x$ is contained in some triad $\Gamma_{x}$. If $\Gamma_{x}$ is disjoint from $\Psi_{1} \cup \Psi_{2}$, then $M \backslash d$ contains three pairwise disjoint triads and we contradict 3.2.1.8. Similarly, if $\Gamma_{x}$ meets both $\Psi_{1}$ and $\Psi_{2}$, there must be three 
pairwise disjoint triads, and if $\left|\Gamma_{x} \cap\left(\Psi_{1} \cup \Psi_{2}\right)\right|=1$ we again get the same contradiction with the use of 3.2.1.9. The only other situation to consider is when $\left|\Gamma_{x} \cap\left(\Psi_{1} \cup \Psi_{2}\right)\right|=2$, and where, up to an arbitrary labelling of the tridents, $\Gamma_{x} \subseteq \Psi_{1} \cup\{x\}$. By 3.2.1.4, there exists a 4-element circuit $C$ in $M$ which contains $d$ as well as two elements from $\Psi_{2}$. But then, at least one of the triads contained in $\Psi_{1} \cup\{x\}$ is disjoint from $C$ and so we arrive a contradiction by orthogonality in the matroid $M$. Consequently we may now assume that $E(M \backslash d)=\Psi_{1} \cup \Psi_{2}$. If $r^{*}(M \backslash d) \geq 4$, then

$$
\lambda\left(\Psi_{1}\right) \leq r_{M \backslash d}^{*}\left(\Psi_{1}\right)+r_{M \backslash d}^{*}\left(\Psi_{2}\right)-4=2
$$

which contradicts the fact that $M \backslash d$ is internally 4-connected. Therefore it must be the case that $r^{*}(M \backslash d)=3$. Let $\Psi_{1}=\left\{x_{1}, x_{2}, \alpha, y_{1}, y_{2}\right\}$ where $\left\{x_{1}, x_{2}, \alpha\right\}$ and $\left\{y_{1}, y_{2}, \alpha\right\}$ are triads, and consider the matroid $M \backslash d / x_{1}, x_{2}$. By virtue of the fact that $r^{*}(M \backslash d)=3$, any potential 2-separation $(P, Q)$ of $M \backslash d / x_{1}, x_{2}$ must have the property that $\left\{x_{1}, x_{2}\right\} \subseteq \mathrm{cl}_{M \backslash d}^{*}(P)$ or $\left\{x_{1}, x_{2}\right\} \subseteq$ $\mathrm{cl}_{M \backslash d}^{*}(Q)$. But this contradicts the fact that $M \backslash d$ is 3 -connected. So $M \backslash d / x_{1}, x_{2}$ is 3 -connected and there exists a 4-element circuit $C$ in $M$ with $\left\{x_{1}, x_{2}, d\right\} \subseteq C$. If $\Psi_{2} \cap C=\emptyset$ then we get a contradiction by orthogonality. So $\Psi_{2} \cap C$ is a singleton. But now $\left\{y_{1}, y_{2}, \alpha\right\}$ is a triad of $M \backslash d$ which is disjoint from $C$ in $M$, again providing a contradiction by orthogonality between the circuit $C$ and the cocircuit $\left\{y_{1}, y_{2}, \alpha, d\right\}$ of $M$.

3.2.1.11. Let $\Psi_{1}$ and $\Psi_{2}$ be two tridents of $M \backslash d$. Then $\left|\Psi_{1} \cap \Psi_{2}\right| \neq 1$.

Proof. Assume otherwise. By 3.2.1.9 we know that no element of $M \backslash d$ is contained in more than two triads, and so we may assume that $\Psi_{1} \cup \Psi_{2}=$ $\left\{t_{1}, \ldots, t_{9}\right\}$ where $\left\{t_{1}, t_{2}, t_{3}\right\},\left\{t_{3}, t_{4}, t_{5}\right\},\left\{t_{5}, t_{6}, t_{7}\right\}$ and $\left\{t_{7}, t_{8}, t_{9}\right\}$ are triads. As $|E(M)|>10$, there exists some $x \in E(M \backslash d)-\left(\Psi_{1} \cup \Psi_{2}\right)$ where $x$ is contained in a triad $\Gamma_{x}$. If $\Gamma_{x}$ is disjoint from $\Psi_{1} \cup \Psi_{2}$, then we contradict 3.2.1.8. If $\left|\Gamma_{x} \cap\left(\Psi_{1} \cup \Psi_{2}\right)\right|=1$, we contradict 3.2.1.10. So $\left|\Gamma_{x} \cap\left(\Psi_{1} \cup \Psi_{2}\right)\right|=2$ and we may assume up to an arbitrary choice of labelling that $\Gamma_{x}=\left\{x, t_{1}, t_{9}\right\}$. If $E(M \backslash d)-\left(\Psi_{1} \cup \Psi_{2} \cup\{x\}\right)$ is non-empty, then $M \backslash d$ must contain another triad. 
But no more triads can be placed relative to our already identified structure without giving either three disjoint triads, two disjoint tridents or an element contained in three distinct triads. So $E(M \backslash d)=\Psi_{1} \cup \Psi_{2} \cup\{x\}$. Consider the matroid $M \backslash d / t_{4}, t_{6}$. If we can show that this matroid is 3-connected, then $M$ must contain a 4 -element circuit $C$ which itself contains $\left\{t_{4}, t_{6}, d\right\}$, which would give the now-more-than-familiar contradiction to orthogonality in the matroid $M$. So, suppose that $(P, Q)$ is indeed a 2-separation of $M \backslash d / t_{4}, t_{6}$. A quick check of orthogonality against the various triads of $M \backslash d$ reveals that $M \backslash d$ cannot possibly contain any 4-element circuits which contain both $t_{4}$ and $t_{6}$. So $M \backslash d / t_{4}, t_{6}$ is free from parallel pairs and we may freely apply Lemma 1.2 .6 to the 2 -separation $(P, Q)$. Without loss of generality, $t_{5} \in Q$. As $t_{4} \in \mathrm{cl}_{M \backslash d / t_{6}}^{*}\left(\left\{t_{3}, t_{5}\right\}\right)$ and $M \backslash d / t_{6}$ is 3 -connected, we are forced to have $t_{3} \in P$. Similarly, it must be that $t_{7} \in P$. Consequently, the triads $\left\{t_{1}, t_{2}, t_{3}\right\}$ and $\left\{t_{7}, t_{8}, t_{9}\right\}$ must both have at least two elements in $P$, and taking the coclosure of $P$, we may assume that $\left\{t_{1}, t_{2}, t_{3}, t_{7}, t_{8}, t_{9}\right\} \subseteq P$. But now $Q \subseteq\left\{t_{5}, x\right\}$ and we contradict the fact that $(P, Q)$ is a 2-separation in a matroid which contains no non-trivial parallel or series classes. Thus $M \backslash d / t_{4}, t_{6}$ must be 3 -connected and the result follows.

3.2.1.12. Let $\Psi_{1}$ and $\Psi_{2}$ be two tridents of $M \backslash d$. Then $\left|\Psi_{1} \cap \Psi_{2}\right| \neq 3$.

Proof. Assume otherwise. From 3.2.1.9, each element of $M \backslash d$ is in at most two triads, and it follows that $\Psi_{1} \cap \Psi_{2}$ must itself be a triad. As $|E(M)|>10$, there exists at least three more elements $\delta, \mu$ and $\zeta \in E(M \backslash d)$ each contained in a corresponding triad $\Gamma_{\delta}, \Gamma_{\mu}$ and $\Gamma_{\zeta}$. By 3.2.1.8 none of these triads are disjoint from $\Psi_{1} \cup \Psi_{2}$. Combining this fact with 3.2.1.9 and 3.2.1.11, it follows that each of $\Gamma_{\delta}, \Gamma_{\mu}$ and $\Gamma_{\zeta}$ meets both $\Psi_{1}-\Psi_{2}$ and $\Psi_{2}-\Psi_{1}$. But this implies that some element of $\Psi_{1} \cup \Psi_{2}$ is contained in at least three triads, which contradicts 3.2.1.9.

3.2.1.13. Let $\Psi_{1}$ and $\Psi_{2}$ be two tridents of $M \backslash d$. Then $\left|\Psi_{1} \cap \Psi_{2}\right| \neq 4$.

Proof. Assume otherwise. As no point of $M \backslash d$ is in three triads and 
$|E(M \backslash d)| \geq 10$, there must exist a triad $\Gamma_{1}$ which is disjoint from $\Psi_{1} \cup \Psi_{2}$, and again, as $|E(M \backslash d)| \geq 10$, there exists yet another triad $\Gamma_{2}$. If $\Gamma_{2}$ is also disjoint from $\Psi_{1} \cup \Psi_{2}$, then we would either have two disjoint tridents or three disjoint triads, both of which are contradictory. So $\left|\Gamma_{2} \cap\left(\Psi_{1} \cup \Psi_{2}\right)\right| \in\{1,2\}$. In such a situation we once again obtain the contradiction that $M \backslash d$ contains 3 disjoint triads unless $\left|\Gamma_{2} \cap\left(\Psi_{1} \cup \Psi_{2}\right)\right|=1$ and $\left|\Gamma_{2} \cap \Gamma_{1}\right|=1$. The exceptional case contradicts Lemma 3.2.1.11.

The matroid $M \backslash d$ must contain at least four distinct triads by 3.2.1.2. By 3.2.1.5 and 3.2.1.8 then, $M \backslash d$ must contain a trident $\Psi_{1}$. If $\Psi_{1}$ is the only trident in $M \backslash d$, then every triad not contained in $\Psi_{1}$ must be disjoint from every other triad of $M \backslash d$. But from the size of the ground set of $M \backslash d$, we know that there are at least two other such triads, and so such a situation contradicts 3.2.1.8. Therefore there must exist a second trident $\Psi_{2}$. Combining the preceeding four results 3.2.1.10, 3.2.1.11, 3.2.1.12 and 3.2.1.13 we are forced into a situation where $\left|\Psi_{1} \cap \Psi_{2}\right|=2$. But by 3.2.1.9, no element can be in three distinct triads and an elementary check of the remaining possible permutations reveals that by considering an alternate labelling of the elements, $\Psi_{1} \cup \Psi_{2}$ is now the union of two distinct tridents whose intersection has size three. This final contradiction completes the proof of 3.2.1 and our arguments in this section. 


\section{Chapter 4}

\section{Troublesome 3-Separating Sets}

Now we are prepared to commence our detailed examination into the exact nature of 3-separating sets which themselves yield no detachable pair.

\subsection{To a More Structured 3-Separating Set}

We begin by identifying a specific substructure that troublesome 3-separating sets necessarily adhere to. This structure will in turn give us information about precisely what is preventing detachable pairs from existing.

Lemma 4.1.1. Let $M$ be a 3-connected matroid with an element $d$ such that $M \backslash d$ is 3-connected. Let $(Y, E(M \backslash d)-Y)$ be an exact 3-separation of $M \backslash d$ such that $|Y| \geq 4$. Assume that $\mathrm{fc}_{M}(Y \cup\{d\})$ contains no triangles or triads and contains no detachable pair. Then there is a subset $X$ of $Y$ having the following properties.

(i) $|X| \geq 4$ and $\lambda_{M \backslash d}(X)=2$.

(ii) $M \backslash d, x$ is 3-connected up to series pairs for all $x \in X$.

(iii) $M \backslash d / x$ is 3-connected for all $x \in X$. 
Proof. Assume that the lemma fails. Choose $X \subseteq Y$ that is minimal with respect to the properties that $|X| \geq 4$ and $\lambda_{M \backslash d}(X)=2$. Let $W=E(M \backslash d)-$ $X$. We prove that the claims of the lemma hold for $X$. By definition, (i) is satisfied. We next prove that $X$ satisfies property (ii). Assume otherwise. Let $x$ be an element of $X$ such that $M \backslash d, x$ is not 3-connected up to series pairs. Let $(P,\{x\}, Q)$ be a cyclic 3-separation of $M \backslash d$.

4.1.1.1. $P \cap W \neq \emptyset \neq Q \cap W$

Proof. Suppose that $Q \cap W=\emptyset$. Then $Q \cup\{x\} \subseteq X$. But then, by the minimality of $X$, we must have $Q \cup\{x\}=X$ as $Q$ is exactly 3-separating. As $Q$ must contain a circuit, this now implies that $X-\{x\}$ is a contradictory triangle. So $Q \cap W \neq \emptyset$ and similarly, $P \cap W \neq \emptyset$.

Without loss of generality we may assume that $|W \cap P| \geq 2$.

4.1.1.2. We may assume that $|X \cap Q|=2$.

Proof. As $|W \cap P| \geq 2$, we have $\lambda_{M \backslash d}(X \cap Q) \leq 2$. If $|X \cap Q|=1$, then $|W \cap Q| \geq 2$, so that $\lambda_{M \backslash d}(X \cap P)=2$ and $|X \cap P| \geq 2$. It follows that, by perhaps switching labels we may assume that $|X \cap Q| \geq 2$.

If $|X \cap Q|>2$, then the set $(X \cap Q) \cup\{x\}$ contradicts the minimality of the choice of $X$. Hence $|X \cap Q|=2$.

4.1.1.3. $|X \cap P|=2$.

Proof. Note that if $|W \cap Q|=1$ then $Q$ is a triangle in $M \backslash d$. So $|W \cap Q| \geq 2$ and, by uncrossing, $\lambda(X \cap P) \leq 2$. Suppose now that $|X \cap P|=1$ with $X \cap P=\{t\}$. Then, as $M \backslash d / t$ is 3 -connected, it must be that $t \in \mathrm{cl}^{*}((X \cap$ $Q) \cup\{x\})$. This, together with the fact that $(X \cap Q) \cup\{x\}$ is a triad of $M \backslash d$, gives $X$ as a 4-element cosegment in $M \backslash d$. As $\operatorname{fcl}(Y \cup\{d\})$ contains no triads, $d$ must block every triad in $X$. But then $M^{*} \mid(X \cup\{d\}) \cong U_{3,5}$ and by Lemma 2.2.5, fcl $(Y \cup\{d\})$ contains a detachable pair. So we may assume that $|X \cap P|>1$. If $|X \cap P|>2$, the set $(X \cap P) \cup\{x\}$ contradicts the minimality of $X$. 
Let $X \cap P=\left\{p_{1}, p_{2}\right\}$ and $X \cap Q=\left\{q_{1}, q_{2}\right\}$.

4.1.1.4. $X$ is closed in $M \backslash d$.

Proof. Suppose $c \in \operatorname{cl}(X)-X$. By symmetry we can assume that $c \in P$. As $(P,\{x\}, Q)$ is cyclic, and $\operatorname{fcl}(Y \cup\{d\})$ contains no triangles, we have $|W \cap Q| \geq 2$. Thus $|E(M)-(X \cup P)| \geq|E(M)-((X \cup\{c\}) \cup P)| \geq 2$ and so, by uncrossing, $\lambda(X \cap P)=\lambda((X \cap P) \cup\{c\})=2$. It follows that $c \in \operatorname{cl}(X \cap P)$; contradicting the fact that $\operatorname{fcl}_{M}(Y \cup\{d\})$ contains no triangles.

4.1.1.5. X contains no 4-element circuits.

Proof. Assume otherwise. Up to symmetry there are two possibilities. Say $\left\{p_{1}, p_{2}, x, q_{1}\right\}$ is a circuit. Then, as $\left\{p_{1}, p_{2}, x\right\}$ and $\left\{x, q_{1}, q_{2}\right\}$ are triads in $M \backslash d$, it follows that $\left(p_{1}, p_{2}, x, q_{1}, q_{2}\right)$ is a flan. Certainly $\left\{p_{1}, p_{2}, x, q_{1}, q_{2}, d\right\}$ is not a flan, and so by Lemma 2.4.5, $\operatorname{fcl}_{M}\left(\left\{p_{1}, p_{2}, x, q_{1}, q_{2}, d\right\}\right)$ contains a contradictory detachable pair. The other possibility is that $\left\{p_{1}, p_{2}, q_{1}, q_{2}\right\}$ is a circuit. Evidently $r(X)=4$ and it follows easily that $\left(\left\{p_{1}, p_{2}, q_{1}, q_{2}\right\}, W \cup\right.$ $\{x\})$ is a 3 -separation of $M$. But then the 3 -separating set $\left\{p_{1}, p_{2}, q_{1}, q_{2}\right\}$ contradicts the minimality of the choice of $X$.

It now follows from 4.1.1.5 and Lemma 2.3.1, that $f c l(Y \cup\{d\})$ contains a detachable pair, contradicting the assumption that no such pair exists. We conclude that property (ii) holds. Finally, we consider (iii). Suppose $x \in X$ is such that $M \backslash d / x$ is not 3 -connected. Let $(P,\{x\}, Q)$ be a 3-separation of $M \backslash d$ with $x \in \operatorname{cl}(P) \cap \operatorname{cl}(Q)$. Assume without loss of generality that $|W \cap P| \geq 2$. Then $\lambda_{M \backslash d}(X \cap Q) \leq 2$. If $|X \cap Q|=2$, then $(X \cap Q) \cup\{x\}$ is either a triangle or a triad of $M \backslash d$. By hypothesis, $(X \cap Q) \cup\{x\}$ is not a triangle. Also $x \in \operatorname{cl}(P)$ and $P \subseteq E(M \backslash d)-((X \cap Q) \cup\{x\})$. Hence $(X \cap Q) \cup\{x\}$ is not a triad. Therefore $|X \cap Q| \neq 2$ so that $|X \cap Q|=1$. If $|W \cap Q|=1$, then similar arguments show that $Q \cup\{x\}$ is a triangle, and, as two elements of this triangle are contained in $X$, the triangle is certainly in the full closure of $Y \cup\{d\}$; contradicting the hypothesis that there are no 
such triangles. Hence $|W \cap Q| \geq 2$. We may now apply the earlier argument to deduce that $|X \cap P|=1$. Hence $|X|=3$, contradicting the assumption that $|X| \geq 4$. We have established (iii) and the lemma is complete. 


\subsection{The Triad Case}

The previous section gives us a 3 -separating set in $M \backslash d$ that has some reasonably strong structure. The 3 -separating set $X$ that we found either contains a triad or not. In this section we examine in more detail the case where $X$ does indeed contain a triad. To simplify the organisation we call the main result a theorem.

Theorem 4.2.1. Let $M$ be a 3-connected matroid with an element $d$ such that $M \backslash d$ is 3-connected. Let $X$ be a 3-separating set of $M \backslash d$ with $|X| \geq 4$ having the following properties:

(i) $\operatorname{co}(M \backslash d, x)$ is 3-connected for all $x \in X$,

(ii) $M \backslash d / x$ is 3-connected for all $x \in X$,

(iii) $\operatorname{fcl}_{M}(X \cup\{d\})$ contains no triads or triangles,

(iv) $E(M \backslash d)-X$ is non-sequential in $M \backslash d$, and

(v) X contains a triad.

Then either:

(a) $\operatorname{fcl}(X \cup\{d\})$ contains a detachable pair, or

(b) $\mathrm{fcl}_{M \backslash d}(X) \cup\{d\}$ is a fully closed twisted 3-separator of $M$, or

(c) $\operatorname{fcl}_{M \backslash d}(X) \cup\{d\}$ is a fully closed partial spider-like 3 -separator of $M$, or

(d) $\operatorname{fcl}_{M \backslash d}(X) \cup\{d\}$ is a fully closed spike-like 3-separator of $M$.

Throughout this section we assume that we are under the hypotheses of Theorem 4.2.1. We also assume that the theorem fails, so that $\mathrm{fcl}_{M}(X \cup\{d\})$ does not contain a detachable pair, and the set $\mathrm{fcl}_{M \backslash d}(X) \cup\{d\}$ is not a fully closed twisted 3-separator of $M$, a fully closed partial spider-like 3-separator of $M$, or a fully closed spike-like 3-separator of $M$. 
Lemma 4.2.2. Any triad of $M \backslash d$ contained in $X$ is fully closed.

Proof. Say $\Gamma$ is a triad of $M \backslash d$ contained in $X$. If $x \in \operatorname{cl}_{M \backslash d}(\Gamma)$, then, $x$ is in a triad $\{x, y, z\}$, as otherwise $\{d, x\}$ is a detachable pair. Evidently we may assume $y \in \Gamma$ and it now follows that $\Gamma \cup\{x, z\}$ is a 5-element set which can be ordered as a maximal flan in $M \backslash d$. But now applying Lemma 3.1.3, one of (a), (b) or (c) must hold. Thus $\Gamma$ is closed. Say $x \in \operatorname{cl}^{*}(\Gamma)$. Then $d$ blocks every triad of the coline $\Gamma \cup\{x\}$ so that $M^{*} \mid(\Gamma \cup\{x, d\}) \cong U_{3,5}$. This contradicts Lemma 2.2.5.

Lemma 4.2.3. $X$ is fully closed in $M \backslash d$.

Proof. Assume otherwise. Then there is a set $Y$ containing $X$ and an element $z$ such that $Y$ and $Y \cup\{z\}$ are 3-separating in $M \backslash d$ and $Y \cup\{z\}$ is fully closed. Let $W=E(M \backslash d)-(Y \cup\{z\})$. We know that $W$ is non-empty as $E(M \backslash d)-X$ is non-sequential. The element $z$ is either in $\operatorname{cl}_{M \backslash d}(Y)$ or $\mathrm{cl}_{M \backslash d}^{*}(Y)$. In the former case $M \backslash d, z$ is 3 -connected unless $z$ is in a triad. But a triad containing $z$ must meet both $Y$ and $W$. This implies that $Y$ is not fully closed. So in this first case, we either find a detachable pair in $Y \cup\{z\}$ or contradict the assumption that $Y \cup\{z\}$ is fully closed. Assume that the latter case holds; that is, assume that $z \in \mathrm{cl}^{*}(Y)$.

4.2.3.1. $M \backslash d / z, x$ is 3 -connected for all $x \in X$.

Proof. Take any $x \in X$. We know that $M \backslash d / x$ is 3-connected. By Bixby's Lemma, $M \backslash d / z, x$ is 3 -connected up to parallel pairs. Thus, if $M \backslash d / z, x$ is not 3 -connected there is a 4-element circuit $C$ of $M \backslash d$ containing $x$ and $z$. As $M \backslash d / x$ is 3-connected, $x \notin \operatorname{cl}(E(M \backslash d)-X)$, and so it must be that $|C \cap X| \geq 2$. Similarly, $z \in \mathrm{cl}_{M \backslash d}^{*}(W)$ so that $|C \cap W|=1$. But now we contradict the fact that $Y \cup\{z\}$ is fully closed.

It follows from 4.2.3.1 that every element of $X$ is in a triangle with $d$ in $M / z$, as otherwise $\{z, x\}$ is a detachable pair for some $x \in X$. Let $T=\left\{t_{1}, t_{2}, t_{3}\right\} \subseteq X$ be a triad of $M \backslash d$. This is fully closed in $M \backslash d$ by 
Lemma 4.2.2. It is now clear that there are distinct elements $s_{1}, s_{2}, s_{3}$ with $\left\{s_{1}, s_{2}, s_{3}\right\} \cap\left\{t_{1}, t_{2}, t_{3}\right\}=\emptyset$ such that $\left\{d, t_{1}, s_{1}\right\},\left\{d, t_{2}, s_{2}\right\}$ and $\left\{d, t_{3}, s_{3}\right\}$ are triangles of $M / z$. As every triangle of $M / z$ containing $d$ meets $T$ by virtue of the fact that $T \cup\{d\}$ is a cocircuit in $M / z$, these are the only triangles containing $d$. Thus $X \subseteq\left\{t_{1}, t_{2}, t_{3}, s_{1}, s_{2}, s_{3}\right\}$. As $T$ is fully closed in $M \backslash d$, at least two elements of $\left\{s_{1}, s_{2}, s_{3}\right\}$ belong to $X$. So we may now assume without loss of generality that $\left\{s_{1}, s_{2}\right\} \subseteq X$.

4.2.3.2. There are distinct elements $u_{1}, u_{2}, \notin\left\{s_{1}, s_{2}, s_{3}, t_{1}, t_{2}, t_{3}\right\}$ such that $\left\{s_{1}, t_{1}, u_{1}\right\}$ and $\left\{s_{2}, t_{2}, u_{2}\right\}$ are triads of $M \backslash d$.

Proof. Consider $s_{1}$. Certainly $s_{1}$ is in a triad $S_{1}$ of $M \backslash d$, as otherwise $\left\{d, s_{1}\right\}$ is a detachable pair. Note that if $\left\{t_{1}, t_{2}, s_{1}, s_{2}\right\}$ were independent in $M \backslash d$, then, as $\left\{t_{1}, t_{2}, s_{1}, s_{2}, d, z\right\}$ has rank 4 in $M$, this would imply that $z \in \mathrm{cl}_{M \backslash d}\left(\left\{t_{1}, t_{2}, s_{1}, s_{2}\right\}\right)$; contradicting the fact that $z \in \mathrm{cl}_{M \backslash d}^{*}(W)$. Thus $\left\{t_{1}, t_{2}, s_{1}, s_{2}\right\}$ is a circuit in $M \backslash d$. Similarly $\left\{t_{2}, t_{3}, s_{2}, s_{3}\right\}$ and $\left\{t_{1}, t_{3}, s_{1}, s_{3}\right\}$ are circuits. We use orthogonality of the triad $S_{1}$ with these circuits frequently in what follows. By Lemma 2.3.2, we may assume that $\left\{s_{1}, s_{2}, s_{3}\right\}$ is not a triad. Suppose that $s_{2} \in S_{1}$. Then, by orthogonality and the fact that $\left\{s_{1}, s_{2}, s_{3}\right\}$ is not a triad, it must be the case that $\left\{s_{1}, s_{2}, t_{3}\right\}$ is a triad. But then $\left\{s_{1}, s_{2}, t_{1}, t_{2}\right\}$ is 2 -separating in $M \backslash d, t_{3}$. But $t_{3} \in X$ and we have contradicted the fact that this matroid is 3-connected up to series pairs. Similarly, $s_{3} \notin S_{1}$. If $\left|S_{1} \cap T\right|=2$, then $S_{1} \cup T$ is a cosegment of $M \backslash d$, all of whose triads are blocked by $d$. This does not happen by the dual of Lemma 2.2.5. The only remaining way to meet the requirements imposed by orthogonality is to have $S_{1} \cap T=\left\{t_{1}\right\}$. The fact that there exists $u_{1} \notin\left\{s_{1}, s_{2}, s_{3}, t_{1}, t_{2}, t_{3}\right\}$ such that $\left\{s_{1}, t_{1}, u_{1}\right\}$ is a triad of $M \backslash d$ follows immediately. Similarly, $s_{2}$ is contained in such a triad $\left\{s_{2}, t_{2}, u_{2}\right\}$. We need to prove that $u_{1} \neq u_{2}$. Assume otherwise. Then $\left\{s_{1}, s_{2}, t_{1}, t_{2}\right\}$ contains a cocircuit of $M \backslash d$. But this set is also a circuit. Hence $\lambda_{M \backslash d}\left(\left\{s_{1}, s_{2}, t_{1}, t_{2}\right\}\right)=2$. But $t_{3} \in \mathrm{cl}_{M \backslash d}^{*}\left(\left\{t_{1}, t_{2}\right\}\right)$ and hence $t_{3} \in \operatorname{cl}_{M \backslash d}^{*}\left(\left\{s_{1}, s_{2}, t_{1}, t_{2}\right\}\right)$. Thus $\left\{s_{1}, s_{2}, t_{1}, t_{2}\right\}$ is 2-separating in $M \backslash d, t_{3}$ contradicting the fact that $M \backslash d, t_{3}$ is 3 -connected up to series pairs. 
It follows that $u_{1}$ and $u_{2}$ are distinct.

By 4.2.3.2 we may assume that $u_{1} \neq z$. We have $u_{1} \in \mathrm{cl}_{M \backslash d}^{*}(X)$, so that $\operatorname{co}\left(M / z \backslash d, u_{1}\right)$ has a 2-separation. Hence by Bixby's Lemma, $M \backslash d / z, u_{1}$ is 3connected up to parallel pairs. Now the argument of 4.2.3.1 may be repeated to prove that $M \backslash d / z, u_{1}$ is 3-connected. But, as we have seen, the only triangles of $M / z$ which contain $d$ are $\left\{d, s_{1}, t_{1}\right\},\left\{d, s_{2}, t_{2}\right\}$, and $\left\{d, s_{3}, t_{3}\right\}$. As $u_{1} \notin\left\{s_{1}, s_{2}, s_{3}, t_{1}, t_{2}, t_{3}\right\}$, it follows that $d$ cannot go into parallel with any element in $M / z, u_{1}$ and thus that $M / z, u_{1}$ is 3 -connected, giving a detachable pair in $\mathrm{fcl}_{M}(X \cup\{d\})$. The lemma follows from this contradiction.

Lemma 4.2.4. Every triad of $M \backslash d$ that meets $X$ is contained within $X$.

Proof. Let $S=\left\{s_{1}, s_{2}, s_{3}\right\}$ be a triad of $M \backslash d$ with $s_{1} \in X$. By Lemma 4.2.3, $|S \cap X| \neq 2$. Suppose that $S \cap X=\left\{s_{1}\right\}$. Now if $X$ is independent in $M \backslash d$, then either $X$ contains triads of $M$, or $X \cup\{d\}$ is a coline of $M$ containing at least five elements. Both of these situations are contradictory, and so we may assume that $X$ contains a circuit. As $s_{1} \in \mathrm{cl}^{*}(E(M \backslash d)-X), X$ itself cannot be a circuit, so that $X-\left\{s_{1}\right\}$ must contain a circuit. This implies that $\left(X-\left\{s_{1}\right\}, E(M \backslash d)-X\right)$ is a 2-separation in $\operatorname{co}\left(M \backslash d, s_{1}\right)$.

Lemma 4.2.5. Let $S \subseteq X, T \subseteq X$ be triads of $M \backslash d$ where $S \neq T$ and $S \cap T \neq \emptyset$. Then $|S \cap T|=1, r_{M \backslash d}(S \triangle T)=4$, and $\lambda_{M \backslash d}(S \cup T)>2$.

Proof. If $|S \cap T|=2$, then $S \cup T$ is a coline of $M \backslash d$. As $d$ blocks every triad, it follows that $M^{*} \mid(S \cup T \cup\{d\}) \cong U_{3,5}$, and, by Lemma 2.2.5, that $M$ contains a detachable pair. So $S \cap T$ is a singleton. Let $\{u\}=S \cap T$. If $r_{M \backslash d}(S \triangle T)=3$, then $\operatorname{co}(M \backslash d, u)$ has a 2-separation, contrary to property (i). Thus $r_{M \backslash d}(S \triangle T)=4$ Finally, suppose that $\lambda_{M \backslash d}(S \cup T)=2$. If $S \cup T$ contains a circuit of size four, then as $r_{M \backslash d}(S \triangle T)=4$, this circuit must contain either $S$ or $T$. But then either $S$ or $T$ is not closed, and this is a contradiction to Lemma 4.2.2. Otherwise, no such circuit exists. The fact that $S \cup T$ is closed in $M \backslash d$ follows from the fact that both $S$ and $T$ are closed, 
and so now by Lemma 2.3.1, $M$ must contain a contradictory detachable pair. Thus we deduce that $\lambda_{M \backslash d}(S \cup T)>2$.

Lemma 4.2.6. Let $R \subseteq X, S \subseteq X$, and $T \subseteq X$ be distinct triads of $M \backslash d$. Then at least one element of $\{S \cap T, S \cap R, R \cap T\}$ is non-empty.

Proof. Assume that $R=\left\{r_{1}, r_{2}, r_{3}\right\}, S=\left\{s_{1}, s_{2}, s_{3}\right\}$ and $T=\left\{t_{1}, t_{2}, t_{3}\right\}$ are pairwise disjoint. By Lemma 4.2.2, $S$ is fully closed in $M \backslash d$. So if $S$ were to be contained in a 4-element fan of $M / r_{1}$, then, up to labelling, $\left\{r_{1}, r_{2}, s_{1}, s_{2}\right\}$ must be a circuit of $M \backslash d$. However, by Lemma 2.3.2, $\sqcap(R, S)=$ 1 , and so $S$ is not contained in a 4 -element fan of $M / r_{3}$. Thus, up to a potential relabelling of the elements of $R$, the triad $S$ is not contained in a 4element fan in $M / r_{1}$. Now by Tutte's Triangle Lemma, we may assume that both $M \backslash d / r_{1}, s_{1}$ and $M \backslash d / r_{1}, s_{2}$ are 3-connected, and so there exist triangles $\left\{d, s_{1}, \alpha\right\}$ and $\left\{d, s_{2}, \beta\right\}$ in $M / r_{1}$. It must be that $\alpha, \beta \in T$, as otherwise $T$ would be a triad in $M / r_{1}$ and hence in $M$. So we may assume that $\left\{d, s_{1}, t_{1}\right\}$ and $\left\{d, s_{2}, t_{2}\right\}$ are triangles in $M / r_{1}$. It follows that $\left\{s_{1}, s_{2}, t_{1}, t_{2}\right\}$ is a circuit, and, as $\sqcap(S, T)=1$; a fact which follows again from Lemma 2.3.2, we have $r(S \cup T)=5$, implying that $t_{3}$ cannot be in a triangle with $d$. Applying Lemma 1.2.19, we see that $M / r_{1}, s_{3}$ must be 3 -connected.

Lemma 4.2.7. Let $R \subseteq X, S \subseteq X$ and $T \subseteq X$ be triads of $M \backslash d$ where $|S \cap T|=1$. Then $R \cap(S \cup T) \neq \emptyset$.

Proof. Let $S=\left\{s_{1}, s_{2}, u\right\}$ and $T=\left\{t_{1}, t_{2}, u\right\}$. Assume that $R \cap S=\emptyset=$ $R \cap T$, and let $Z=E(M \backslash d)-(S \cup T)$.

4.2.7.1. $\sqcap(Z, S)=\sqcap\left(Z,\left\{s_{1}, s_{2}\right\}\right)=1=\sqcap(Z, T)=\sqcap\left(Z,\left\{t_{1}, t_{2}\right\}\right)$

Proof. If $r(Z)=r(M)-3$, then by Lemma 1.2.18, $S \cup T$ is a 5-point coline. As $d$ blocks every triad of $M \backslash d$, it follows that $M^{*} \mid(S \cup T \cup\{d\}) \cong U_{3,6}$, which yields a contradiction to Lemma 2.2.5. Hence $r(Z)=r(M)-2$ and it 
follows from the definition of local connectivity that

$$
\begin{aligned}
\sqcap(Z, S) & =r(Z)+r(S)-r(Z \cup S) \\
& =(r(M)-2)+3-r(M)=1=\sqcap\left(Z,\left\{s_{1}, s_{2}\right\}\right) .
\end{aligned}
$$

Similarly, we obtain $\sqcap(Z, T)=\sqcap\left(Z,\left\{t_{1}, t_{2}\right\}\right)=1$.

4.2.7.2. Suppose $z \in Z$. If $S$ is not closed in $M \backslash d / z$, then $M \backslash d / z$ has a triangle containing $\left\{s_{1}, s_{2}\right\}$. Similarly, if $T$ is not closed in $M \backslash d / z$, then $M \backslash d / z$ has a triangle containing $\left\{t_{1}, t_{2}\right\}$.

Proof. This is a straightforward consequence of 4.2.7.1.

Let $R=\left\{r_{1}, r_{2}, r_{3}\right\}$.

4.2.7.3. We may assume that $S$ is closed in $M \backslash d / r_{1}$.

Proof. If $T$ is closed, we may simply switch the labels of $S$ and $T$. So suppose that neither $S$ nor $T$ is closed in $M \backslash d / r_{1}$. Then it follows from 4.2.7.2 that $M \backslash d / r_{1}$ contains triangles $\left\{t_{1}, t_{2}, r_{2}\right\}$ and $\left\{s_{1}, s_{2}, r_{3}\right\}$ up to labelling. Observe now that $\left\{s_{1}, s_{2}\right\}$ is not contained in a triangle in $M \backslash d / r_{2}$. By switching the labels on $r_{1}$ and $r_{2}$, we may now assume that $S$ is closed in $M \backslash d / r_{1}$ as required.

Note that $S \cup\{d\}$ and $T \cup\{d\}$ are cocircuits in the matroid $M / r_{1}$, and $S$ is a closed triad in $M \backslash d / r_{1}$. By Tutte's Triangle Lemma, there are two elements from $\left\{s_{1}, s_{2}, u\right\}$ that can be contracted from $M \backslash d / r_{1}$ whilst maintaining 3connectivity. Suppose to begin with that $M \backslash d / r_{1}, s_{1}$ and $M \backslash d / r_{1}, s_{2}$ are 3-connected. As $M$ contains no detachable pair, $M / r_{1}$ must contain two triangles, one containing $\left\{s_{1}, d\right\}$, the other containing $\left\{s_{2}, d\right\}$. As $T \cup\{d\}$ is a cocircuit, each of these triangles must meet $T$. If $\left\{s_{1}, d, t_{1}\right\}$ and $\left\{s_{2}, d, t_{2}\right\}$ are triangles, we obtain the contradiction that $r_{M / r_{1}}\left(s_{1}, s_{2}, t_{1}, t_{2}\right)=3$. So without loss of generality, $\left\{s_{1}, d, u\right\}$ and $\left\{s_{2}, d, t_{1}\right\}$ are triangles. But then we have the contradiction that $r\left(\left\{s_{1}, s_{2}, u, t_{1}\right\}\right)=3$. The other possibility up to 
symmetry is that $M \backslash d / r_{1}, s_{1}$ and $M \backslash d / r_{1}, u$ are 3-connected. Here $M / r_{1}$ has a triangle containing $\left\{d, s_{1}\right\}$ and another containing $\{d, u\}$. By orthogonality, the triangle containing $d$ and $s_{1}$ must intersect the set $\left\{t_{1}, t_{2}, u\right\}$. If this point of intersection is $u$, then these two triangles are identical, and it follows that $\left\{s_{1}, s_{2}, d, u\right\}$ is exactly 3 -separating in $M / r_{1}$. But now $\operatorname{co}\left(M / r_{1} \backslash s_{2}\right)$ is not 3connected, so that by Bixby's Lemma, $M / r_{1}, s_{2}$ is 3 -connected up to parallel pairs. By 4.2.7.3, this matroid cannot contain parallel pairs, implying that $\left\{r_{1}, s_{2}\right\}$ is a contradictory detachable pair. So we may assume without loss of generality that $\left\{d, s_{1}, t_{1}\right\}$ is a triangle. Applying Lemma 1.2.20, $s_{2}$ must also be in a triangle which meets both $\{d, u\}$ and $\left\{t_{1}, t_{2}\right\}$, but all possible permutations of this triangle give $r_{M / r_{1}}\left(\left\{s_{1}, s_{2}, u, t_{1}, t_{2}\right\}\right)=4$, implying that $S \cup T$ is 3-separating in $M \backslash d$; an implication which contradicts Lemma 4.2.5.

Lemma 4.2.8. Let $R \subseteq X, S \subseteq X$ and $T \subseteq X$ be triads of $M \backslash d$ where $S \cap T=\{u\}$. Then $R \cap(S \cup T) \neq\{u\}$.

Proof. Suppose contrary to the claim of the lemma that $R=\left\{r_{1}, r_{2}, u\right\}$ where $S=\left\{s_{1}, s_{2}, u\right\}$ and $T=\left\{t_{1}, t_{2}, u\right\}$. Consider $M \backslash u, d / t_{1}$. This matroid is 3 -connected up to series pairs; a fact which follows from our underlying assumption that $M \backslash u, d$ is. Both $u$ and $d$ must block the series pairs of $M \backslash u, d / t_{1}$, and we have that $\left\{r_{1}, r_{2}, u, d\right\}$ and $\left\{s_{1}, s_{2}, u, d\right\}$ are cocircuits of $M / t_{1}$. If there exists a series pair $P$ of $M \backslash u, d / t_{1}$ which is neither $\left\{r_{1}, r_{2}\right\}$ nor $\left\{s_{1}, s_{2}\right\}$, then it follows that either $X \cup\{d\}$ is a spike-like 3separator, or else, up to an arbitrary choice of labelling, there exists some $\alpha \in E(M \backslash d)-(R \cup S \cup T)$ such that $\left\{\alpha, r_{2}, s_{2}\right\}$ is a triad. The first possibility contradicts our assumption that (d) doesn't hold, while the second possibility contradicts Lemma 4.2.7. Thus we may assume that $M \backslash u, d / t_{1}$ is 3-connected up to the series pairs $\left\{r_{1}, r_{2}\right\}$ and $\left\{s_{1}, s_{2}\right\}$. Now applying Lemma 1.2.20, $M / t_{1}$ has triangles $\left\{r_{1}, s_{1}, u\right\}$ and $\left\{r_{2}, s_{2}, d\right\}$, giving circuits $\left\{r_{1}, s_{1}, t_{1}, u\right\}$ and $\left\{r_{2}, s_{2}, t_{1}, d\right\}$ in $M$.

4.2.8.1. The set $\left\{r_{1}, r_{2}, s_{1}, s_{2}, t_{1}, t_{2}\right\}$ is either a circuit or independent in $M$. 
Proof. If $\left\{r_{2}, s_{2}, t_{2}\right\}$ is a triangle in $\operatorname{co}(M \backslash u, d)$ then $\left\{r_{1}, r_{2}, s_{1}, s_{2}, t_{1}, t_{2}\right\}$ is a circuit in $M$. Otherwise, $\left\{r_{1}, r_{2}, s_{1}, s_{2}, t_{1}, t_{2}\right\}$ must be independent.

Consider now the matroid $M \backslash u, d / t_{2}$. Once more applying Lemma 1.2.20, we see that $M$ contains circuits $\left\{t_{2}, u, r_{i}, s_{j}\right\}$ and $\left\{t_{2}, d, r_{i^{\prime}}, s_{j^{\prime}}\right\}$, where $\left\{i^{\prime}\right\}=$ $\{1,2\}-\{i\}$ and $\left\{j^{\prime}\right\}=\{1,2\}-\{j\}$. We identify the first of these circuits.

4.2.8.2. $\left\{t_{2}, u, r_{2}, s_{2}\right\}$ is a circuit.

Proof. Suppose $j=1$. Then either $\left\{t_{2}, u, r_{1}, s_{1}\right\}$ or $\left\{t_{2}, u, r_{2}, s_{1}\right\}$ is a circuit. In either of these cases we have $\left\{t_{1}, t_{2}\right\} \subseteq \operatorname{cl}\left(\left\{r_{1}, r_{2}, s_{1}, u\right\}\right)$, thus $r\left(\left\{t_{1}, t_{2}, r_{1}, r_{2}, s_{1}\right\}\right) \leq 4$, which contradicts 4.2.8.1. Similarly $i=1$ and so $\left\{t_{2}, u, r_{2}, s_{2}\right\}$ is a circuit.

It now follows that $\left\{t_{2}, d, r_{1}, s_{1}\right\}$ is the second of the aforementioned circuits. Now consider the matroid $M / r_{1}$. Applying Lemma 1.2.20, we arrive at the conclusion that $M / r_{1}, s_{2}$ is 3-connected unless $s_{2}$ is in a triangle with exactly one element from each of $\{d, u\}$ and $\left\{t_{1}, t_{2}\right\}$. But the existence of this triangle implies that $s_{2} \in \mathrm{cl}_{M / r_{1}}\left(\left\{s_{1}, d, u, t_{1}, t_{2}\right\}\right)$ meaning in particular that $r_{M / r_{1}}\left(s_{1}, s_{2}, t_{1}, t_{2}\right)=3$ when we know that $\left\{s_{1}, s_{2}, t_{1}, t_{2}\right\}$ is independent.

Lemma 4.2.9. Let $R \subseteq X, S \subseteq X$ and $T \subseteq X$ be distinct triads of $M \backslash d$ where $S \cap T=\left\{t_{3}\right\}$. Then $S \cap R \neq \emptyset \neq T \cap R$.

Proof. Suppose the lemma fails. We may assume then that $T \cap R=\emptyset$ so that by Lemma 4.2.7, $|S \cap R|=1$. Thus we have triads $T=\left\{t_{1}, t_{2}, t_{3}\right\}$, $R=\left\{r_{1}, r_{2}, r_{3}\right\}$ and $S=\left\{s, r_{3}, t_{3}\right\}$. We begin by showing that $X$ consists of no more than these three triads.

4.2.9.1. $X=R \cup S \cup T$.

Proof. Suppose that $z \in X-(R \cup S \cup T)$. Certainly $z$ must be in a triad $\Gamma \subseteq X$ of $M \backslash d$ by Lemma 4.2.4. Note that as $M \backslash d$ does not contain a 4point coline, $\Gamma$ intersects each of the triads $R, S$ and $T$ at no more than a 
single point. By Lemma 4.2.8 then, we have that $\left\{r_{3}, t_{3}\right\} \cap \Gamma=\emptyset$. Suppose that $|\Gamma \cap(R \cup S \cup T)|=1$. If $\Gamma \cap(R \cup S \cup T)=\{s\}$, then $\Gamma, R$ and $T$ are disjoint, contradicting Lemma 4.2.6. Otherwise we again contradict Lemma 4.2.7. So $|\Gamma \cap(R \cup S \cup T)|=2$. It now follows from this together with another application of Lemma 4.2.7 that $s \notin \Gamma$, as otherwise either $R$ or $T$ would be disjoint from $\Gamma$. Thus we may assume without loss of generality that $\Gamma=\left\{z, r_{2}, t_{2}\right\}$. If there exists some other $z^{\prime} \in X-(R \cup S \cup T \cup\{z\})$, with corresponding triad $\Gamma^{\prime} \subseteq X$, then again we have $\Gamma^{\prime} \cap S=\emptyset$. But now we are forced into a contradiction at the hands of either Lemma 4.2 .6 or Lemma 4.2.7. Therefore $X=R \cup S \cup T \cup\{z\}$.

4.2.9.1.1. $S$ is closed in $M \backslash d / z$.

Proof. Assume otherwise. By Lemma 1.2.8 and Lemma 4.2.3, $\mathrm{cl}_{M^{*} / d \backslash z}^{*}(S) \subseteq$ $X$. Without loss of generality, $t_{2} \in \mathrm{cl}_{M^{*} / d \backslash z}^{*}(S)$. By orthogonality with the triangle $R$ of $M^{*} / d \backslash z$, the set $S \cup\left\{t_{2}\right\}$ is not a cocircuit in $M^{*} / d \backslash z$ and so $\left\{s, t_{2}, t_{3}\right\}$ must be a triad in this matroid. Therefore

$$
t_{1} \in \mathrm{cl}_{M^{*} / d \backslash z}\left(E\left(M^{*} / d \backslash z\right)-\left(S \cup\left\{t_{2}\right\}\right)\right)
$$

and thus if $S \cup\left\{t_{2}\right\}$ is not coclosed in $M^{*} / d \backslash z$, we may assume without loss of generality that $r_{2} \in \mathrm{cl}_{M^{*} / d \backslash z}^{*}\left(S \cup\left\{t_{2}\right\}\right)$. But now we have another triad $\left\{s, r_{2}, r_{3}\right\}$ in $M^{*} / d \backslash z$, and this implies that

$$
\begin{aligned}
& E\left(M^{*} / d \backslash z\right)-\left(\left\{s, r_{2}, r_{3}\right\} \cup\left\{s, t_{2}, t_{3}\right\}\right) \\
& =\left(E\left(M^{*} / d \backslash z\right)-X\right) \cup\left\{r_{1}, t_{1}\right\}
\end{aligned}
$$

is a flat of rank at most $r\left(M^{*} / d \backslash z\right)-2$. However $E\left(M^{*} / d \backslash z\right)-X$ is exactly 3-separating in $M^{*} / d \backslash z$ and has rank $r\left(M^{*} / d \backslash z\right)-2$. Thus

$$
\begin{aligned}
\left\{r_{1}, t_{1}\right\} & \subseteq \operatorname{cl}_{M^{*} / d \backslash z}\left(E\left(M^{*} / d \backslash z\right)-X\right) \\
& \subseteq \operatorname{cl}_{M^{*} / d}\left(E\left(M^{*} / d\right)-X\right) .
\end{aligned}
$$

But $t_{1}$ is not in a triangle with every element of $X$ in $M^{*} / d$, and consequently $\left(X-\left\{t_{1}\right\}, E\left(M^{*} / d\right)-X\right)$ is a non-trivial 2-separation in the 3-connected matroid $\operatorname{si}\left(M^{*} / d, t_{1}\right)=\operatorname{co}\left(M \backslash d, t_{1}\right)$. So $S \cup\left\{t_{2}\right\}$ must be coclosed in $M^{*} / d \backslash z$. 
We now proceed to show that $M^{*} / d \backslash z, r_{1}$ and $M^{*} / d \backslash z, r_{2}$ are 3 -connected. Suppose that $(P, Q)$ is a 2-separation of $M^{*} / d \backslash z, r_{2}$. By orthogonality, any triad of $M^{*} / d \backslash z$ which contains $r_{2}$ must also contain two elements from $S$. Such a situation contradicts the fact we have just established that $S \cup\left\{t_{2}\right\}$ is coclosed in $M^{*} / d \backslash z$. Therefore $M^{*} / d \backslash z, r_{2}$ does not contain any series pairs and so by Lemma 1.2.6, we may freely apply the full closure operator on the 2-separation $(P, Q)$. Without loss of generality, we may assume that $S \subseteq P$. As $t_{2} \in \mathrm{cl}_{M^{*} / d \backslash z}^{*}(S)$, we may also assume that $t_{2} \in P$, and consequently that $t_{1} \in P$ also. So we have $S \cup T \subseteq P$. But both $S \cup T$ and $X-\left\{r_{2}, z\right\}$ are exactly 3 -separating in $M^{*} / d \backslash r_{2}, z$, while their symmetric difference is $\left\{r_{1}\right\}$. This implies that $r_{1} \in \mathrm{cl}_{M^{*} / d \backslash r_{2}, z}^{(*)}(S \cup T)$ and so we may also assume that $r_{1} \in P$. But then $r_{2} \in \operatorname{cl}_{M^{*} / d \backslash z}(P)$ which implies the existence of a 2 -separation in the 3 -connected matroid $M^{*} / d \backslash z$. Thus $M^{*} / d \backslash r_{2}, z$ must be 3-connected. An almost identical argument shows that $M^{*} / d \backslash r_{1}, z$ is also 3-connected.

As $M$ does not contain any detachable pairs, $M^{*}$ must have a 4 -element cocircuit which contains the set $\left\{r_{1}, z, d\right\}$ as well as a 4-element cocircuit which contains the set $\left\{r_{2}, z, d\right\}$. By orthogonality with the circuits $S \cup\{d\}$ and $T \cup\{d\}$ of $M^{*}$, the only viable possibility is that both $\left\{r_{1}, z, d, t_{3}\right\}$ and $\left\{r_{2}, z, d, t_{3}\right\}$ are cocircuits of $M^{*}$. But now $M$ has a restriction isomorphic to $U_{3,5}$ and we contradict Lemma 2.2.5. We are left to conclude that $S$ must be closed in $M \backslash d / z$ as required.

Combining 4.2.9.1.1 with Tutte's Triangle Lemma and using symmetry between $r_{3}$ and $t_{3}$ we now have two possible scenarios. Either both of $M \backslash d / z, r_{3}$ and $M \backslash d / z, t_{3}$ are 3 -connected, or both of $M \backslash d / z, s$ and $M \backslash d / z, t_{3}$ are 3-connected. Assume for the moment that the first of these possibilities holds. Then $M / z$ must contain triangles $\left\{d, r_{3}, \alpha\right\}$ and $\left\{d, t_{3}, \beta\right\}$.

It is possible that these two triangles are equal, where $\alpha=t_{3}$ and $\beta=r_{3}$. Suppose that this is the case. Every triad of $M / z \backslash d$ is blocked by $d$ in $M / z$ and so the element $s$ is certainly not in any triad in $M / z$. Now the 
triangle $\left\{r_{3}, t_{3}, d\right\}$ is 2-separating in $M / z$ and thus by Bixby's Lemma, $M / z, s$ must be 3 -connected up to parallel pairs. However by 4.2.9.1.1, s cannot be in a triangle in $M / z$ and thus $M / z, s$ must be 3-connected; giving a detachable pair in $M$. Therefore we may assume that the triangles $\left\{d, r_{3}, \alpha\right\}$ and $\left\{d, t_{3}, \beta\right\}$ of $M / z$ are distinct.

This now gives a cocircuit $\left\{r_{3}, t_{3}, \alpha, \beta\right\}$ in $M^{*} / d \backslash z$. By orthogonality with the two triangles $R$ and $T$ of $M^{*} / d \backslash z$, we may then assume without loss of generality that this cocircuit is $\left\{r_{3}, t_{3}, r_{2}, t_{2}\right\}$. Consider now the matroid $M^{*} / d \backslash z, s$. By 4.2.9.1.1, $S$ is coclosed in $M^{*} / d \backslash z$ and so $s$ cannot be in any triad of $M^{*} / d \backslash z$ as any such triad would necessarily contain two elements from $S$. Therefore we know that $M^{*} / d \backslash z, s$ does not have any series pairs. Suppose however that it does have some 2-separation $(P, Q)$. Note that we may apply Lemma 1.2.6 freely. We may assume that $r_{3} \in P$ and $t_{3} \in Q$, for otherwise we would have $s \in \operatorname{cl}_{M^{*} / d \backslash z}(P)$ or $s \in \operatorname{cl}_{M^{*} / d \backslash z}(Q)$ which would clearly be a contradictory situation within the 3 -connected matroid $M^{*} / d \backslash z$. For precisely the same reasons, we then must have $R \subseteq P$ and $T \subseteq Q$. Let $Y=E\left(M^{*} / d \backslash z, s\right)-X$. As $|Y| \geq 3$, we may assume that $|P \cap Y| \geq 2$. By submodularity of the connectivity function we have

$$
\begin{aligned}
& \lambda_{M^{*} / d \backslash z, s}(P \cap Y)+\lambda_{M^{*} / d \backslash z, s}(P \cup Y) \\
\leq & \lambda_{M^{*} / d \backslash z, s}(P)+\lambda_{M^{*} / d \backslash z, s}(Y) \leq 1+2=3 .
\end{aligned}
$$

So either $P \cap Y$ or $P \cup Y$ must be 2-separating in $M^{*} / d \backslash z, s$. The first possibility implies that $(X-\{z, s\}) \cup Q$ is 2-separating, but since $s \in \mathrm{cl}_{M^{*} / d \backslash z}(X-\{z, s\})$, this gives a 2-separation in the 3-connected matroid $M^{*} / d \backslash z$ by virtue of the fact that $|P \cap Y| \geq 2$. So $P \cup Y$ must be 2-separating in $M^{*} / d \backslash z, s$. Now $r_{M^{*} / d \backslash z, s}(Y)=r(M)-2$ and we know that $r_{1} \notin \mathrm{cl}_{M^{*} / d \backslash z, s}(Y)$, for otherwise $\operatorname{si}\left(M^{*} / d, r_{1}\right)$ would fail to be 3 -connected. So $r_{M^{*} / d \backslash z, s}\left(Y \cup\left\{r_{1}\right\}\right)=r(M)-1$. As $r_{2}$ is an element of the cocircuit $\left\{r_{2}, t_{2}, r_{3}, t_{3}\right\}$ of $M^{*} / d \backslash z$ and this cocircuit is disjoint from $Y \cup\left\{r_{1}\right\}$, it then follows that $r_{M^{*} / d \backslash z, s}\left(Y \cup\left\{r_{1}, r_{2}\right\}\right)=r(M)$. So $Y \cup\left\{r_{1}, r_{2}\right\}$ spans $M^{*} / d \backslash z$ and thus $s \in \operatorname{cl}_{M^{*} / d \backslash z}(P \cup Y)$, which again exposes a 2-separation in the 
3-connected matroid $M^{*} / d \backslash z$. We are left to conclude that $M^{*} / d \backslash z, s$ must be 3-connected.

Returning to our application of Tutte's Triangle Lemma, we may now safely assume that both $M^{*} / d \backslash z, s$ and $M^{*} / d \backslash z, t_{3}$ are 3 -connected. This gives triangles $\{d, s, \alpha\}$ and $\left\{d, t_{3}, \beta\right\}$ in $M / z$. Just as argued previously, if these two triangles are identical, then we find that $M / z, r_{3}$ is 3 -connected. So the triangles are distinct, and this leads us to the existence of a 4-element cocircuit $\left\{s, t_{3}, \alpha, \beta\right\}$ in $M^{*} / d \backslash z$. Checking orthogonality against triangles $R, S$ and $T$ readily shows that this cocircuit must be $\left\{s, t_{3}, t_{2}, t_{1}\right\}$. However now $T$ is a 2 -separating triangle in $M^{*} / d \backslash z, s$, a matroid which we know to be 3-connected. This final contradiction completes the proof of the lemma.

With 4.2.9.1 in hand, we henceforth assume that $X=R \cup S \cup T$. The next step is to show that the triad $S$ of $M \backslash d$ has the following property:

4.2.9.2. For all $z \in\left\{r_{1}, r_{2}, t_{1}, t_{2}\right\}$, the triad $S$ is not closed in $M \backslash d / z$.

Proof. Suppose that $S$ is closed in $M \backslash d / t_{2}$. Then by Tutte's Triangle Lemma and using symmetry between $s$ and $t_{3}$, we either have that both of $M \backslash d / t_{2}, r_{3}$ and $M \backslash d / t_{2}, t_{3}$ are 3-connected, or both of $M \backslash d / t_{2}, s$ and $M \backslash d / t_{2}, t_{3}$ are 3connected.

4.2.9.2.1. The element $d$ is not in a triangle of $M / t_{2}$ with two elements from $S$.

Proof. If $d$ is indeed in such a triangle, let $q$ be the element from $S$ which is not contained in this triangle, and note that $M / t_{2} \backslash q$ is not 3-connected up to series pairs. By Bixby's Lemma, $M / t_{2}, q$ must be 3-connected up to parallel pairs, but the existence of a parallel pair would imply that $S \cup\{d\}$ is not closed in $M / t_{2}$, and hence that $S$ is not closed in $M \backslash d / t_{2}$.

4.2.9.2.2. We may assume that $\left\{d, t_{3}, r_{1}\right\}$ is a triangle in $M / t_{2}$. 
Proof. We know for sure that $M \backslash d / t_{2}, t_{3}$ is 3 -connected, and so there must exist a triangle $\left\{t_{3}, d, \alpha\right\}$ in $M / t_{2}$. As $d$ blocks the triad $R$ of $M \backslash d$, the set $R \cup\{d\}$ is a cocircuit in $M / t_{2}$, and so by orthogonality, it follows that $\alpha \in R$. By 4.2.9.2.1, $\alpha \neq r_{3}$, and so up labelling, we may assume that $\left\{d, t_{3}, r_{1}\right\}$ is a triangle in $M / t_{2}$.

We now know that $\lambda_{M / t_{2}}\left((X \cup\{d\})-\left\{t_{2}\right\}\right)=2$. Let $W=E(M)-(X \cup$ $\{d\})$. The following result eliminates one of the possibilities that arose from our application of Tutte's Triangle Lemma.

4.2.9.2.3. $\{d, s\}$ is not contained in a triangle in $M / t_{2}$.

Proof. If it is, then as per the argument of 4.2.9.2.2, we have that $\{d, s, \alpha\}$ is a triangle in $M / t_{2}$ where $\alpha \in\left\{r_{1}, r_{2}\right\}$. By 4.2.9.2.1, $\alpha \neq r_{1}$ and this triangle is $\left\{d, s, r_{2}\right\}$; which gives a circuit $\left\{r_{1}, r_{2}, s, t_{3}\right\}$ in $M / t_{2}$. As $S$ and $R$ are triads of $M / t_{2} \backslash d$, it follows that $r_{M / t_{2} \backslash d}\left(W \cup\left\{t_{1}\right\}\right) \leq r\left(M / t_{2} \backslash d\right)-2$. But now $r_{M / t_{2} \backslash r_{3}}\left(W \cup\left\{t_{1}\right\}\right) \leq r\left(M / t_{2} \backslash r_{3}\right)-2$ so that $\lambda_{M / t_{2} \backslash r_{3}}\left(\left\{r_{1}, r_{2}, s, t_{3}, d\right\}\right)=1$. By Bixby's Lemma, $M / t_{2}, r_{3}$ must be 3-connected up to parallel pairs, hence $r_{3}$ must be contained in some triangle of $M / t_{2}$. As both $R \cup\{d\}$ and $S \cup\{d\}$ are cocircuits of $M / t_{2}$, it must be that $d$ is also contained in this triangle. The triangle cannot be a subset of $\left\{r_{1}, r_{2}, r_{3}, s, t_{3}, d\right\}$, and so must either contain $t_{1}$ or meet $W$. We know from Lemma 4.2 .3 that $X$ is fully closed in $M \backslash d$. Hence by Lemma 1.2.7, $X \cup\{d\}$ is fully closed in $M$. Therefore the triangle in question cannot meet $W$, so the triangle must be $\left\{d, r_{3}, t_{1}\right\}$.

Note now that both $W$ and $W \cup\left\{t_{1}\right\}$ are exactly 3 -separating in $M / t_{2}$, and thus $t_{1} \in \operatorname{cl}_{M / t_{2}}(X \cup\{d\}) \cap \mathrm{cl}_{M / t_{2}}(W)$. Suppose that $M / t_{2} \backslash r_{2}$ is not 3 -connected. Then, as $M / t_{2}$ has no series pairs, $M / t_{2} \backslash r_{2}$ has a nontrivial 2-separation $(P, Q)$. By Lemma 1.2.6, we may assume that the triad $\left\{d, r_{1}, r_{3}\right\} \subseteq P$. As $\left\{d, t_{3}, r_{1}\right\}$ is a triangle in $M / t_{2} \backslash r_{2}$, we may assume that $t_{3} \in P$, and, as $S \cup\{d\}$ is a cocircuit, that $s \in P$ also. But $r_{2} \in \operatorname{cl}_{M / t_{2}}(\{s, d\})$ and so then $\left(P \cup\left\{r_{2}\right\}, Q\right)$ is a 2-separation in the 3 -connected matroid $M / t_{2}$. So $M / t_{2} \backslash r_{2}$ must be 3 -connected. By 
Bixby's Lemma, $M / t_{2} \backslash r_{1}, t_{1}$ is now 3 -connected unless $t_{1}$ is in a triad $\Gamma$ of $M / t_{2} \backslash r_{2}$ with $\Gamma \cap W \neq \emptyset \neq \Gamma \cap\left\{r_{1}, r_{3}, d, s, t_{3}\right\}$. This would imply that $(X \cup\{d\})-\left\{t_{2}, r_{2}\right\}$ is not fully closed in $M / t_{2} \backslash r_{2}$. But as we have seen, $X \cup\{d\}$ is fully closed in $M$, and thus $(X \cup\{d\})-\left\{r_{2}, t_{2}\right\}$ must certainly be fully closed in $M / t_{2} \backslash r_{2}$. Therefore $M / t_{2} \backslash r_{2}, t_{1}$ is 3 -connected.

It now follows that $M$ has a cocircuit $C^{*}=\left\{t_{1}, t_{2}, r_{2}, \alpha\right\}$. As $X \cup\{d\}$ is fully closed in $M, \alpha \in\left\{r_{1}, r_{3}, s, t_{3}, d\right\}$. Recall that $C_{T}=T \cup\{d\}, C_{R}=R \cup\{d\}$ and $C_{S}=S \cup\{d\}$ are cocircuits in $M$. If $\alpha \in C_{S}$, then $r_{1} \notin C^{*} \cup C_{S} \cup C_{T}$ so that $E(M)-\left(C^{*} \cup C_{S} \cup C_{T} \cup C_{R}\right)$ is a flat of rank at most $r(M)-4$. If $\alpha \notin C_{s}$, then $\alpha=r_{1}$, which gives $s \notin C^{*} \cup C_{R} \cup C_{T}$; again implying that $E(M)-\left(C^{*} \cup C_{S} \cup C_{T} \cup C_{R}\right)=W$ is a flat of rank at most $r(M)-4$. But $W$ is exactly 3 -separating in $M$ and so we contradict the fact that $M$ is 3-connected.

With 4.2.9.2.3 in hand, we know that $M \backslash d / t_{2}, s$ cannot be 3-connected. Therefore $M \backslash d / t_{2}, r_{3}$ must be 3 -connected. It follows that there exists a triangle $\left\{r_{3}, d, \gamma\right\}$ in $M / t_{2}$.

4.2.9.2.4. $\left\{r_{2}, r_{3}, d\right\}$ is not a triangle in $M / t_{2}$.

Proof. Assume otherwise. Then $\left\{r_{1}, r_{2}, r_{3}, t_{3}\right\}$ is a circuit of $M / t_{2}$ by 4.2.9.2.2. The set $R \cup\{d\}$ is a rank-3 cocircuit of $M / t_{2}$ with $t_{3} \in$ $\mathrm{cl}_{M / t_{2}}(R \cup\{d\})$. So $R \cup\left\{d, t_{3}\right\}$ is exactly 3-separating in $M / t_{2}$. Suppose that $r_{M / t_{2}}\left(\left\{r_{1}, r_{2}, s, t_{3}\right\}\right)=4$. Then $s \in\left(\mathrm{cl}_{M / t_{2}}^{*}\left(R \cup\left\{d, t_{3}\right\}\right)-\mathrm{cl}_{M / t_{2}}\left(R \cup\left\{d, t_{3}\right\}\right)\right)$. Hence

$$
s \in\left(\mathrm{cl}_{M / t_{2}}^{*}\left(W \cup\left\{t_{1}\right\}\right)-\mathrm{cl}_{M / t_{2}}\left(W \cup\left\{t_{1}\right\}\right)\right)
$$

which gives $\lambda_{M / t_{2} \backslash s}\left(R \cup\left\{d, t_{3}\right\}\right)=1$. By Bixby's Lemma, si $\left(M / t_{2}, s\right)$ must be 3 -connected. As $M$ does not contain a detachable pair, $s$ must be in a triangle $\{\alpha, s, \beta\}$ of $M / t_{2}$ with $\alpha \in\left(X \cup\left\{d, t_{3}\right\}\right)$ and $\beta \in\left(W \cup\left\{t_{1}\right\}\right)$. As $R \cup\{d\}$ and $S \cup\{d\}$ are cocircuits, we are forced by orthogonality to have 
$\alpha=d$. This contradicts 4.2.9.2.3. We may now assume that $\left\{r_{1}, r_{2}, s, t_{3}\right\}$ is a circuit in $M / t_{2}$. Here $r_{M / t_{2}}(R \cup S \cup\{d\})=3$. But now

$$
\begin{aligned}
& \lambda_{M / t_{2}}(R \cup S \cup\{d\}) \\
= & r_{M / t_{2}}(R \cup S \cup\{d\})+r_{M / t_{2}}^{*}(R \cup S \cup\{d\})-|R \cup S \cup\{d\}|=1
\end{aligned}
$$

and we contradict the fact that $M / t_{2}$ is 3 -connected.

Now $\gamma \notin\left\{r_{1}, t_{3}\right\}$ as $M / t_{2}$ cannot contain a 4-point line by Lemma 2.2.5. Therefore the only possibility that does not contradict the fact that $X-\left\{t_{2}\right\}$ is fully closed in $M \backslash d / t_{2}$ is that $\gamma=t_{1}$. Note that $r_{M / t_{2}}\left(\left(X-\left\{t_{2}\right\}\right) \cup\{d\}\right)=4$.

4.2.9.2.5. If $s$ is contained in a triangle $T_{s}$ of $M / t_{2}$, then $T_{s}=\left\{s, t_{1}, t_{3}\right\}$.

Proof. Suppose that $T_{s}$ is a triangle containing $s$. By 4.2.9.2.3, $d \notin T_{s}$. The triangle $T_{s}$ lifts up to a circuit $T_{s} \cup\left\{t_{2}\right\}$ of $M$, while $T \cup\{d\}=\left\{t_{1}, t_{2}, t_{3}, d\right\}$ is a cocircuit of $M$. Therefore $T_{s} \cap\left\{t_{1}, t_{3}\right\} \neq \emptyset$ by orthogonality. Similarly, as $\left\{r_{3}, s, t_{3}, d\right\}$ is a cocircuit, $T_{s} \cap\left\{r_{3}, t_{3}\right\} \neq \emptyset$. So $t_{3} \in T_{s}$. Now, as $X-\left\{t_{2}\right\}$ is fully closed in $M / t_{2}$, the only possibility that does not contradict the fact that $R \cup\{d\}$ is a cocircuit is that $T_{s}=\left\{s, t_{1}, t_{3}\right\}$.

4.2.9.2.6. $\left\{r_{1}, r_{2}, s, t_{3}\right\}$ is a circuit of $M / t_{2}$.

Proof. Assume that $\left\{r_{1}, r_{2}, s, t_{3}\right\}$ is independent in $M / t_{2}$. Suppose that $s$ is in a triangle $T_{s}$ of $M / t_{2}$. Then by 4.2.9.2.5, $T_{s}=\left\{s, t_{1}, t_{3}\right\}$. Suppose also that $r_{2}$ is in a triangle $T_{r_{2}}$ of $M / t_{2}$. By Lemma 1.2.7, $\left(X-\left\{t_{2}\right\}\right) \cup\{d\}$ is fully closed in $M / t_{2}$, so that $T_{r_{2}} \subseteq\left(X-\left\{t_{2}\right\}\right) \cup\{d\}$. By 4.2.9.2.5, $s \notin T_{r_{2}}$. If $d \in T_{r_{2}}$, then it now follows that $M / t_{2}$ contains a 4-point line, contradicting Lemma 2.2.5. As $T_{r_{2}} \cup\left\{t_{2}\right\}$ is a circuit in $M$ and $T \cup\{d\}$ a cocircuit in $M$, it must be that $T_{r_{2}} \cap\left\{t_{1}, t_{3}\right\} \neq \emptyset$. Also, as $R \cup\{d\}$ is a cocircuit, $T_{r_{2}} \cap\left\{r_{1}, r_{3}\right\} \neq \emptyset$. The only possibilities that do not give a contradictory 4-point line in $M / t_{2}$ are that $T_{r_{2}}=\left\{r_{2}, r_{1}, t_{1}\right\}$ or $T_{r_{2}}=\left\{r_{2}, r_{3}, t_{3}\right\}$. But both of these cases contradict our assumption that $r_{M / t_{2}}\left(\left\{r_{1}, r_{2}, s, t_{3}\right\}\right)=4$. Thus if $\left\{r_{1}, r_{2}, s, t_{3}\right\}$ is to be independent in $M / t_{2}$, then at least one of $s$ and $r_{2}$ 
is not in a triangle of $M / t_{2}$. Assume without loss of generality that $s$ is not contained in a triangle. Consider $M / t_{2}, s$. This matroid must now have a non-trivial 2-separation $(P, Q)$. We may freely apply Lemma 1.2.6 here. Hence we may assume that the triangle $\left\{d, t_{3}, r_{1}\right\} \subseteq P$. It now cannot be that $r_{3} \in P$ as otherwise $s \in \mathrm{cl}_{M / t_{2}}^{*}(P)$, which would give a 2-separation $(P \cup\{s\}, Q)$ in $M / t_{2}$. So $r_{3} \in Q$ and thus $t_{1}, r_{2} \in Q$ also as $\left\{d, r_{3}, t_{1}\right\}$ is a triangle and $R \cup\{d\}$ is a cocircuit. But now $d \in \mathrm{cl}_{M / t_{2}, s}(Q)$, hence $r_{1} \in \mathrm{cl}_{M / t_{2}, s}^{*}(Q \cup\{d\})$, and thus $\left(X-\left\{t_{2}, s\right\}\right) \subseteq \mathrm{fcl}_{M / t_{2}, s}(Q)$ so that we may switch to a 2-separation $\left(P^{\prime}, Q^{\prime}\right)$ of $M / t_{2}, s$ in which $\left(X-\left\{t_{2}, s\right\}\right) \cup\{d\} \subseteq P^{\prime}$. But now $s \in \mathrm{cl}_{M / t_{2}}^{*}\left(P^{\prime}\right)$ and $\left(P^{\prime} \cup\{s\}, Q^{\prime}\right)$ is a 2-separation in the 3-connected matroid $M / t_{2}$. We conclude that $\left\{r_{1}, r_{2}, s, t_{3}\right\}$ is a circuit as required.

If $s$ is in a triangle of $M / t_{2}$, then by 4.2.9.2.5, this triangle is $\left\{s, t_{1}, t_{3}\right\}$. But then $\left\{t_{1}, r_{3}, d\right\} \subseteq \operatorname{cl}_{M / t_{2}}\left(\left\{r_{1}, r_{2}, s, t_{3}\right\}\right)$ and by 4.2.9.2.6 we contradict the fact that $r_{M / t_{2}}\left(\left(X-\left\{t_{2}\right\}\right) \cup\{d\}\right)=4$. So $s$ is not in such a triangle, and as $M$ does not contain a detachable pair, $M / t_{2}, s$ must have a non-trivial 2 -separation $(P, Q)$. The set $\left\{t_{3}, r_{1}, r_{2}, d\right\}$ is a 4 -point line in $M / t_{2}, s$, so we may assume that $\left\{t_{3}, r_{1}, r_{2}, d\right\} \subseteq P$. By Lemma 1.2.6, we may also assume that $r_{3} \in P$, and consequently that $t_{1} \in P$ as $R \cup\{d\}$ is a cocircuit of $M / t_{2}, s$, while $\left\{d, r_{3}, t_{1}\right\}$ is a triangle. But now $s \in \mathrm{cl}_{M / t_{2}}^{*}(P)$ giving a 2-separation $(P \cup\{s\}, Q)$ in $M / t_{2}$. We conclude that $S$ cannot be closed in $M \backslash d / t_{2}$. As our choice of $t_{2} \in\left\{r_{1}, r_{2}, t_{1}, t_{2}\right\}$ was arbitrary, the claim now follows.

We require the following result in what follows.

4.2.9.3. If $C_{1} \subseteq X$ and $C_{2} \subseteq X$ are distinct cocircuits of $M^{*} / d$, then $X \subseteq$ $C_{1} \cup C_{2}$.

Proof. Let $C_{1}$ and $C_{2}$ be such cocircuits. Note that $r_{M^{*} / d}\left(E\left(M^{*} / d\right)-X\right)=$ $r\left(M^{*} / d\right)-2$ as $X$ is an exactly 3 -separating set with rank 4 . The set $E\left(M^{*} / d\right)-\left(C_{1} \cup C_{2}\right)$ must now be a flat of rank $r\left(M^{*} / d\right)-2$. Thus if $x \in X-\left(C_{1} \cup C_{2}\right)$ for some $x$, then $x \in \operatorname{cl}_{M^{*} / d}\left(E\left(M^{*} / d\right)-X\right)$. But this implies that $X-\{x\}$ is 2-separating in $\operatorname{si}\left(M^{*} / d, x\right)$; a contradiction. 
As $X$ is fully closed in $M \backslash d$, we know now from 4.2.9.2 that $\operatorname{cl}_{M \backslash d / t_{2}}(S) \cap$ $\left\{r_{1}, r_{2}, t_{1}\right\} \neq \emptyset$. Similarly, $\mathrm{cl}_{M \backslash d / r_{2}}(S) \cap\left\{r_{1}, t_{1}, t_{2}\right\} \neq \emptyset$. Our next result specifies precisely the contents of these intersections.

4.2.9.4. We may assume that both $S \cup\left\{r_{1}, t_{1}\right\}$ and $S \cup\left\{r_{2}, t_{2}\right\}$ contain cocircuits of $M^{*} / d$.

Proof. Suppose that $t_{1} \in \operatorname{cl}_{M \backslash d / t_{2}}(S)$. Then $S \cup\left\{t_{1}\right\}$ contains a circuit in $M \backslash d / t_{2}$. As $r_{3} \in \mathrm{cl}_{M \backslash d / t_{2}}^{*}\left(\left\{r_{1}, r_{2}\right\}\right)$, the triple $\left\{t_{1}, t_{3}, s\right\}$ must be a triangle in $M \backslash d / t_{2}$. Hence $\left\{t_{1}, t_{2}, t_{3}, s\right\}$ is a circuit of $M \backslash d$. But now $T$ is both a circuit and a cocircuit of $M \backslash d / s$ and we contradict the fact that this matroid is 3connected. So $t_{1} \notin \mathrm{cl}_{M \backslash d / t_{2}}(S)$ and we may assume up to a choice of labelling that $r_{2} \in \mathrm{cl}_{M \backslash d / t_{2}}(S)$. Hence $S \cup\left\{r_{2}\right\}$ contains a cocircuit of $M^{*} / d \backslash t_{2}$ so that

$$
\begin{array}{r}
r_{M^{*} / d \backslash t_{2}}\left(E\left(M^{*} / d \backslash t_{2}\right)-\left(S \cup\left\{r_{2}\right\}\right)\right) \\
\leq r\left(M^{*} / d \backslash t_{2}\right)-1 .
\end{array}
$$

If $r_{1} \in \mathrm{cl}_{M \backslash d / t_{2}}(S)$ also, then

$$
r_{1} \notin \operatorname{cl}_{M^{*} / d \backslash t_{2}}\left(E\left(M^{*} / d \backslash t_{2}\right)-\left(S \cup\left\{r_{1}, r_{2}\right\}\right)\right) .
$$

Thus,

$$
r_{M * / d \backslash t_{2}}\left(E\left(M / d \backslash t_{2}\right)-\left(S \cup\left\{r_{1}, r_{2}\right\}\right)\right) \leq r\left(M / d \backslash t_{2}\right)-2 .
$$

But $r_{M / d \backslash t_{2}}\left(S \cup\left\{r_{1}, r_{2}\right\}\right)=3$, implying that $S \cup\left\{r_{1}, r_{2}\right\}$ is 2-separating in the 3-connected matroid $M^{*} / d \backslash t_{2}$. Therefore $\operatorname{cl}_{M \backslash d / t_{2}}(S)=S \cup\left\{r_{2}\right\}$ and so $S \cup\left\{r_{2}, t_{2}\right\}$ contains a cocircuit $C_{2}$ of $M^{*} / d$ as required. Similarly, we obtain $\operatorname{cl}_{M \backslash d / t_{1}}(S)=S \cup\left\{r_{k}\right\}$ for some $k \in\{1,2\}$, and thus we have a cocircuit $C_{1}$ of $M^{*} / d$ contained in $S \cup\left\{t_{1}, r_{k}\right\}$. Suppose that $C_{1}=C_{2}$. Then by orthogonality with the triangle $T$ of $M^{*} / d$, it must be that $C_{1}=C_{2}=\left\{r_{2}, r_{3}, s\right\}$. But this contradicts the fact $M$ contains no triangles. Hence $C_{1}$ and $C_{2}$ must be distinct. By 4.2.9.3 it follows that $k=1$ so that $S \cup\left\{r_{1}, t_{1}\right\}$ also contains a cocircuit of $M^{*} / d$. 
It now follows from 4.2.9.3 and 4.2.9.4 that both $\left\{r_{1}, r_{3}, t_{1}, t_{3}\right\}$ and $\left\{r_{2}, r_{3}, t_{2}, t_{3}\right\}$ are cocircuits in the matroid $M^{*} / d \backslash s$.

4.2.9.5. $S \cup\{d\},\left\{r_{1}, t_{1}, s, d\right\}$ and $\left\{r_{2}, t_{2}, s, d\right\}$ are cocircuits of $M^{*}$.

Proof. Note that if $\Gamma$ is a triad of $M^{*} / d \backslash s$ that meets $X-\{s\}$, then it follows that $\Gamma \subseteq X-\{s\}$. But neither $R$ nor $T$ can be a triad as such a triad would be 2 -separating. So either $|T \cap \Gamma|=1$ or $|R \cap \Gamma|=1$, but now we have a contradiction by orthogonality. Hence $M^{*} / d \backslash s$ must be without such triads and so for each $x \in X-\{s\}$, we know that $M^{*} / d \backslash s, x$ has no series pairs. Consider $M^{*} / d \backslash s, t_{2}$ and suppose that it is not 3-connected. Then it has a non-trivial 2-separation $(P, Q)$. Lemma 1.2.6 will be used freely. We may assume that the triangle $R \subseteq P$. It must now be that $t_{3} \in Q$ as otherwise $(P \cup\{s\}, Q)$ would be 2-separating in $M^{*} / d \backslash t_{2}$, and to avoid a similar contradiction we must have $t_{1} \in Q$ as $\left\{t_{1}, t_{3}, r_{1}, r_{3}\right\}$ is a cocircuit in $M^{*} / d \backslash s, t_{2}$. But now $t_{2} \in \operatorname{cl}_{M^{*} / d \backslash s}(Q)$ and $\left(P, Q \cup\left\{t_{2}\right\}\right)$ is 2separating in $M^{*} / d \backslash s$. Therefore $M^{*} / d \backslash s, t_{2}$ must be 3-connected. Similarly, each of $M^{*} / d \backslash s, t_{1}, M^{*} / d \backslash s, r_{1}$ and $M^{*} / d \backslash s, r_{2}$ are 3-connected. A virtually identical argument also gives that both $M^{*} / d \backslash s, r_{3}$ and $M^{*} / d \backslash s, t_{3}$ are 3connected. Thus we find that $d$ must be in some triangle with every element from $X-\{s\}$ in the matroid $M / s$. As both $\left\{r_{1}, t_{1}, r_{3}, t_{3}\right\}$ and $\left\{r_{2}, t_{2}, r_{3}, t_{3}\right\}$ are circuits in $M / s$, it is readily deduced that the only possible arrangement is that where $\left\{r_{1}, t_{1}, d\right\},\left\{r_{2}, t_{2}, d\right\}$ and $\left\{r_{3}, t_{3}, d\right\}$ are triangles of $M / s$. The result follows.

Observe that $S \cup\{d\}$ is both a circuit and a cocircuit of $M$.

4.2.9.6. $\sqcap_{M^{*}}\left(\left\{r_{3}, t_{3}\right\}, E(M)-(X \cup\{d\})\right)=1$.

Proof. By 4.2.9.5, $\left\{r_{1}, t_{1}, s, d\right\}$ and $\left\{r_{2}, t_{2}, s, d\right\}$ are both cocircuits of $M^{*}$. Therefore $E\left(M^{*}\right)-\left\{r_{1}, r_{2}, t_{1}, t_{2}, d, s\right\}$ is a flat of $M^{*}$ with rank at most $r\left(M^{*}\right)-2$. We know that $r_{M^{*}}(X \cup\{d\})=5$. Thus if

$$
r_{3} \notin \mathrm{cl}_{M^{*}}\left(E\left(M^{*}\right)-\left\{r_{1}, r_{2}, r_{3}, t_{1}, t_{2}, d, s\right\}\right)
$$


then $r_{M^{*}}\left(E\left(M^{*}\right)-(X \cup\{d\})\right) \leq r\left(M^{*}\right)-4$, and we contradict the fact that $M^{*}$ is 3-connected.

Consider the 3 -connected matroid $M^{*} / r_{3}$ and suppose that $\left\{r_{1}, r_{2}, s\right\}$ is not a triangle in this matroid. Let $(P, Q)$ be a 2-separation of $M^{*} / r_{1}, r_{3}$ and note that we may freely apply Lemma 1.2 .6 to this separation as $M^{*} / r_{3}, r_{1}$ has no series nor parallel pairs. We may assume that the triangle $\left\{d, s, t_{3}\right\}$ is contained in $P$. As $\left\{d, s, r_{1}, t_{1}\right\}$ is a cocircuit of $M^{*} / r_{3}$, we must then have $t_{1} \in Q$, for otherwise $\left(P \cup\left\{r_{1}\right\}, Q\right)$ would be 2-separating in $M^{*} / r_{3}$. Furthermore, as we are forced to have $t_{1} \in Q$, we must also have $t_{2} \in Q$ as $t_{1} \in \mathrm{cl}_{M^{*} / r_{1}, r_{3}}\left(\left\{t_{2}, d\right\}\right)$. This necessitates that $r_{2} \in Q$ as well because $\left\{d, s, r_{2}, t_{2}\right\}$ is a cocircuit of $M^{*} / r_{3}$. However we are now left with a situation in which $d \in \mathrm{cl}_{M^{*} / r_{3}}(Q)$ and consequently $r_{1} \in \mathrm{cl}_{M^{*} / r_{3}}^{*}\left(\mathrm{cl}_{M^{*} / r_{3}}(Q)\right)$ which gives $\left(P, Q \cup\left\{d, r_{3}\right\}\right)$ as a 2 -separation in the 3 -connected matroid $M^{*} / r_{3}$. We are left to conclude that $\left\{r_{1}, r_{2}, s\right\}$ must be a triangle of $M^{*} / r_{3}$ and $\left\{r_{1}, r_{2}, r_{3}, s\right\}$ a circuit of $M^{*}$. A corresponding argument shows that $\left\{t_{1}, t_{2}, t_{3}, s\right\}$ is also a circuit of $M^{*}$. By definition,

$$
\begin{array}{r}
\sqcap_{M^{*}}\left(S \cup\{d\}, E\left(M^{*}\right)-(X \cup\{d\})\right) \\
=3+r_{M^{*}}\left(E\left(M^{*}\right)-(X \cup\{d\})\right)-r_{M^{*}}\left(S \cup\{d\} \cup\left(E\left(M^{*}\right)-(X \cup\{d\})\right)\right) .
\end{array}
$$

We have

$$
\begin{aligned}
& r_{M^{*}}\left(\left(E\left(M^{*}\right)-(X \cup\{d\})\right) \cup\left\{r_{3}\right\}\right) \\
& \quad=r_{M^{*}}\left(E\left(M^{*}\right)-(X \cup\{d\})\right)+1,
\end{aligned}
$$

and, because $\left\{r_{1}, t_{1}, s, d\right\}$ is a cocircuit, we then have

$$
\begin{array}{r}
r_{M^{*}}\left(E\left(M^{*}\right)-(X \cup\{d\}) \cup\left\{r_{3}, d\right\}\right) \\
\quad=r_{M^{*}}\left(E\left(M^{*}\right)-(X \cup\{d\})\right)+2 .
\end{array}
$$

This shows that

$$
\begin{array}{r}
r_{M^{*}}\left(\left(E\left(M^{*}\right)-(X \cup\{d\})\right) \cup S \cup\{d\}\right) \\
=r_{M^{*}}\left(E\left(M^{*}\right)-(X \cup\{d\})\right)+2,
\end{array}
$$


and therefore that $\sqcap_{M^{*}}\left(S \cup\{d\}, E\left(M^{*}\right)-(X \cup\{d\})\right)=1$. By performing cocircuit exchange on the cocircuits $\left\{r_{1}, t_{1}, s, d\right\}$ and $\left\{r_{2}, t_{2}, s, d\right\}$ in the matroid $M^{*}$, we see that $\left\{r_{1}, r_{2}, t_{1}, t_{2}, s\right\}$ must contain a cocircuit, and by orthogonality with the quad $S \cup\{d\}$, this cocircuit must be $\left\{r_{1}, r_{2}, t_{1}, t_{2}\right\}$. It follows from this that

$$
\sqcap_{M^{*} / t_{1}}\left(S \cup\{d\}, E\left(M^{*}\right)-(X \cup\{d\})\right)=1 .
$$

But $t_{2} \in \mathrm{cl}_{M^{*} / t_{1}}(S \cup\{d\})$ and $r_{1} \in \mathrm{cl}_{M^{*} / t_{1}}\left(E\left(M^{*} / t_{1}\right)-(X \cup\{d\})\right)$. Hence

$$
\sqcap_{M^{*} / t_{1}}\left(S \cup\left\{d, t_{2}\right\},\left\{r_{1}\right\} \cup\left(E\left(M^{*} / t_{1}\right)-(X \cup\{d\})\right)\right)=1 .
$$

Now if either $r_{2} \in \mathrm{cl}_{M^{*} / t_{1}}(S \cup\{d\})$ or $r_{2} \in \mathrm{cl}_{M^{*} / t_{1}}\left(E\left(M^{*} / t_{1}\right)-(X \cup\{d\})\right)$, then $\operatorname{si}\left(M^{*} / d, t_{1}\right)$ fails to be 3 -connected. Therefore $r_{2}$ must block this potential 2-separation and it follows from Bixby's Lemma together with the fact that $r_{2}$ is not in a triangle of $M^{*} / t_{1}$ that $M^{*} / t_{1}, r_{2}$ must be 3 -connected. This completes the proof of the lemma.

Lemma 4.2.10. Let $R \subseteq X, S \subseteq X$ and $T \subseteq X$ be distinct triads of $M \backslash d$ where $S \cap T=\left\{t_{3}\right\}$. Then $|R \cap(S \cup T)| \neq 2$.

Proof. Assume otherwise. By Lemma 4.2 .9 above, $R$ must intersect both $S$ and $T$. As we have seen, if $M \backslash d$ contains a 4-point coline, then $M^{*}$ must have a restriction isomorphic to $U_{3,5}$, which contradicts Lemma 2.2.5. So no such coline arises and it follows easily from this fact that $t_{3} \notin R$. Thus we have an arrangement of the triads $S, T$ and $R$ of $M \backslash d$ such that $S=\left\{s_{1}, s_{2}, t_{3}\right\}$, $T=\left\{t_{1}, t_{2}, t_{3}\right\}$ and $R=\left\{s_{1}, t_{1}, r\right\}$.

4.2.10.1. $X=R \cup S \cup T$.

Proof. Suppose $x \in X-(R \cup S \cup T)$. As $\operatorname{co}(M \backslash x, d)$ is 3-connected, $x$ must be contained in a triad $\Gamma \subseteq X$. By Lemma 4.2.9, $\Gamma$ must intersect each of $R, S$ and $T$. As $M \backslash d$ cannot contain a 4-point coline, the only way this configuration can arise is if $\Gamma \in\left\{\left\{x, t_{3}, r\right\},\left\{x, s_{1}, t_{2}\right\},\left\{x, t_{1}, s_{2}\right\}\right\}$. Each of these possibilities contradicts Lemma 4.2.8. 
4.2.10.2. For each $x \in X$, no element of $X-\{x\}$ is in a triad of $M^{*} / d \backslash x$.

Proof. Suppose that for some $x \in X, \Gamma$ is a triad of $M^{*} / d \backslash x$ which meets $X-\{x\}$. Then $\Gamma$ lifts to a 4-element cocircuit $C$ of $M^{*} / d$. By orthogonality with the triangles in $X$, we certainly must now have $|C \cap X| \geq 3$. If $|C \cap X|=$ 3 , then $X$ fails to be coclosed in $M^{*} / d$, contradicting Lemma 4.2.3, while if $C \subseteq X$, then there must exist some $z \in X$ such that $z \in \operatorname{cl}_{M^{*} / d}\left(E\left(M^{*} / d\right)-\right.$ $X)$; implying that $\operatorname{si}\left(M^{*} / d, z\right)=\operatorname{co}(M \backslash d, z)$ has a contradictory non-trivial 2-separation.

Combining 4.2.10.2 with Lemma 1.2.14, we now deduce that $M^{*} / d \backslash x_{i}, x_{j}$ is 3 -connected for any $x_{i}, x_{j} \in X$. Thus for any such $x_{i}, x_{j}$, the triple $\left\{d, x_{i}, x_{j}\right\}$ is contained in a 4 -element cocircuit of $M^{*}$. The cocircuit $C_{1}$ containing $\left\{t_{1}, t_{2}, d\right\}$ must have its fourth element in $\left\{s_{1}, s_{2}, t_{3}\right\}$ by orthogonality with the circuit $\left\{s_{1}, s_{2}, t_{3}, d\right\}$. Similarly, the cocircuit $C_{2}$ containing $\left\{t_{1}, r, d\right\}$ has its fourth element in $\left\{s_{1}, s_{2}, t_{3}\right\}$. At least one element from $\left\{s_{1}, s_{2}, t_{3}\right\}$ is in neither $C_{1}$ nor $C_{2}$. It matters not which element for our purposes, and we may assume without loss of generality that $t_{3} \notin\left(C_{1} \cup C_{2}\right)$. But then $E\left(M^{*}\right)-\left(C_{1} \cup C_{2}\right)$ is a flat of rank $r\left(M^{*}\right)-2$ and so $r_{M^{*} / d}\left(E\left(M^{*} / d\right)-\left(C_{1} \cup C_{2}\right)\right)=r\left(M^{*} / d\right)-1$. As $t_{3} \notin\left(C_{1} \cup C_{2}\right)$, this implies that $t_{3} \in \mathrm{cl}_{M^{*} / d}\left(E\left(M^{*} / d\right)-X\right)$, which contradicts the fact that $M^{*} / d$, $t_{3}$ is 3-connected up to parallel pairs.

Proof of Theorem 4.2.1. Let $T \subseteq X$ be a triad. As $|X| \geq 4$, there exists some $x \in X-T$. By Lemma 4.2.4, $x$ is contained in some triad $S=\left\{s_{1}, s_{2}, s_{3}\right\} \subseteq$ $X$. Suppose that the triads $S$ and $T$ intersect. Then by Lemma 4.2.5, we have $|S \cap T|=1$ and $\lambda_{M \backslash d}(S \cup T)>2$. As $X$ is 3-separating, there must exist some $r \in(X-(S \cup T))$. Of course $\operatorname{co}(M \backslash d, r)$ is 3-connected and applying Lemma 4.2.4 we uncover the triad $R \subseteq X$. By Lemma 4.2.9, $R$ must intersect both $S$ and $T$. As $M \backslash d$ cannot, as we have seen, contain a 4-element coline, it must be the case that $|R \cap S|=|R \cap T|=1$. Lemma 4.2.8 together with Lemma 4.2 .10 shows that this is impossible. We are left to conclude 
that $S$ and $T$ are disjoint. If $X-(S \cup T)$ is still non-empty, Lemma 4.2.4 now implies that $X$ must contain three pairwise disjoint triads, but this contradicts Lemma 4.2.6. The only remaining possibility is that $X=S \cup T$. In particular, $S \cup T$ is 3-separating and the only triads contained in $X$ are precisely $S$ and $T$. By Lemma 2.3.2, $\sqcap(S, T) \neq 2$. If $\sqcap(S, T)=0$, then $S \cup T$ is independent and thus is a coline, contradicting the fact that $S$ and $T$ are the only triads of $S \cup T$. So $\sqcap(S, T)=1$. Let $Z=E(M \backslash d)-(S \cup T)$.

4.2.10.3. For all $\left\{s^{\prime}, s^{\prime \prime}\right\} \subseteq S$ and all $\left\{t^{\prime}, t^{\prime \prime}\right\} \subseteq T, \sqcap\left(\left\{s^{\prime}, s^{\prime \prime}\right\}, T\right)=$ $\sqcap\left(\left\{s^{\prime}, s^{\prime \prime}\right\}, Z\right)=\sqcap\left(\left\{t^{\prime}, t^{\prime \prime}\right\}, S\right)=\sqcap\left(\left\{t^{\prime}, t^{\prime \prime}\right\}, Z\right)=0$.

Proof. Suppose $\sqcap\left(\left\{s^{\prime}, s^{\prime \prime}\right\}, T\right)=1$ and let $s^{\prime \prime \prime}=S-\left\{s^{\prime}, s^{\prime \prime}\right\}$. Then $r(T \cup$ $\left.\left\{s^{\prime}, s^{\prime \prime}\right\}\right)=4$ and $r\left(Z \cup\left\{s^{\prime \prime \prime}\right\}\right)=r(Z)+1$ so that $Z \cup\left\{s^{\prime \prime \prime}\right\}$ is 3-separating, implying that $s^{\prime \prime \prime} \in \mathrm{cl}^{*}(Z)$. But then $\left(T \cup\left\{s^{\prime}, s^{\prime \prime}\right\}, Z\right)$ is a 2-separation of $M \backslash d, s^{\prime \prime \prime}$, contradicting our assumption that this matroid is 3-connected up to series pairs. So $\sqcap\left(\left\{s^{\prime}, s^{\prime \prime}\right\}, T\right)=0$. Essentially the same argument shows that $\sqcap\left(\left\{s^{\prime}, s^{\prime \prime}\right\}, Z\right)=0$ and by symmetry we also obtain $\sqcap\left(\left\{t^{\prime}, t^{\prime \prime}\right\}, S\right)=$ $\sqcap\left(\left\{t^{\prime}, t^{\prime \prime}\right\}, Z\right)=0$.

By Lemma 4.2.2, $T$ is closed in $M \backslash d$, and, by 4.2.10.3, it is also closed in $M \backslash d / s_{1}$. So $T$ cannot be contained in a 4 -element fan and by Tutte's Triangle Lemma, we may assume that $M \backslash d / s_{1}, t_{1}$ and $M \backslash d / s_{1}, t_{2}$ are 3connected. As $M$ contains no detachable pairs, we therefore have triangles $\left\{d, t_{1}, \alpha\right\}$ and $\left\{d, t_{2}, \beta\right\}$ and thus a circuit $\left\{t_{1}, t_{1}, \alpha, \beta\right\}$ in $M / s_{1}$ so that $\sqcap_{M / s_{1}}\left(\left\{t_{1}, t_{2}\right\},\{\alpha, \beta\}\right)=1$.

4.2.10.4. $\{\alpha, \beta\} \cap(S \cup T) \neq \emptyset$ and $\{\alpha, \beta\} \neq\left\{s_{2}, s_{3}\right\}$.

Proof. If $\{\alpha, \beta\} \subseteq Z$, then $\sqcap_{M}\left(\left\{t_{1}, t_{2}\right\},\{\alpha, \beta\}\right)=1$, and so $\sqcap\left(\left\{t_{1}, t_{2}\right\}, Z\right) \geq 1$, contradicting 4.2.10.3. If $\{\alpha, \beta\}=\left\{s_{2}, s_{3}\right\}$, then $\sqcap\left(S,\left\{t_{1}, t_{2}\right\} \geq 1\right.$; again a contradiction to 4.2.10.3. 
By 4.2.10.4, we may assume that $\alpha \in\left\{s_{2}, s_{3}\right\}$ and $\beta \in Z$. But now $\beta \in \mathrm{cl}_{M \backslash d / s_{1}}\left(T \cup\left\{s_{2}, s_{3}\right\}\right)$, so that $\beta \in \operatorname{cl}_{M \backslash d}(S \cup T)$, contradicting the fact established in Lemma 4.2.3 that $S \cup T=X$ is fully closed.

This final contradiction completes the proof of Theorem 4.2.1. 


\subsection{The Non-Triad Case}

In this section we deal with the case that our structured 3-separating set $X$ as identified in Lemma 4.1.1 does not contain a triad. As in the previous section, we shall refer to the main result as a theorem for the sake of clarity.

Theorem 4.3.1. Let $M$ be a 3-connected matroid with an element $d$ such that $M \backslash d$ is 3-connected and non-sequential. Let $X$ be a 3-separating set of $M \backslash d$ with $|X| \geq 4$, having the following properties:

(a) $\operatorname{co}(M \backslash d, x)$ is 3-connected for all $x \in X$,

(b) $M \backslash d / x$ is 3-connected for all $x \in X$,

(c) $\operatorname{fcl}_{M}(X \cup\{d\})$ contains no triads or triangles,

(d) $E(M \backslash d)-X$ is non-sequential in $M \backslash d$, and

(e) $X$ contains no triads of $M \backslash d$

Then either:

(i) $\operatorname{fcl}_{M \backslash d}(X) \cup\{d\}$ contains a detachable pair,

(ii) $|X|=4$ and $\operatorname{cl}(X \cup\{d\})$ contains a detachable pair,

(iii) there exists $c \in E(M)-(X \cup\{d\})$ such that $\mathrm{fcl}_{M \backslash d}(X) \cup\{d\}=X \cup\{c, d\}$ is a spike-like 3-separator,

(iv) there exists $c \in E(M)-(X \cup\{d\})$ such that $\mathrm{fcl}_{M \backslash d}(X) \cup\{d\}=X \cup\{c, d\}$ is a partial spider-like 3-separator with associated partition $\{X,\{c, d\}\}$, or

(v) there exists a set $Y \subseteq E(M)-X$ with $d \in Y$ such that fcl $\operatorname{fod}_{M \backslash d}(X) \cup\{d\}=$ $X \cup Y$ is a spider-like 3-separator with associated partition $\{X, Y\}$. 
We shall assume for the remainder of this section that we are under the hypotheses of Theorem 4.3.1 and that $\operatorname{fcl}_{M \backslash d}(X) \cup\{d\}$ does not contain a detachable pair.

Lemma 4.3.2. $E(M \backslash d)-X$ is fully closed.

Proof. If $E(M \backslash d)-X$ is not closed, then there must exist some $x \in X$ such that $M \backslash d / x$ fails to be 3 -connected. As $X$ contains no triads, it must contain a circuit of $M \backslash d$. Suppose $x \in X \cap \operatorname{cl}^{*}(E(M \backslash d)-X)$. Then $X$ cannot be a circuit and so $X-\{x\}$ contains a circuit of $M \backslash d$. But this implies that $\operatorname{co}(M \backslash d, x)$ has a 2-separation $(X-\{x\}, E(M \backslash d)-X)$.

Lemma 4.3.3. In $M \backslash d$, every element of $X$ is in a triad, and every triad that meets $X$ contains exactly one element of $E(M \backslash d)-X$.

Proof. It is clear that every element of $X$ is in a triad, as otherwise $M$ would contain a detachable pair. Let $T$ be a triad that contains some $x \in X$. If $\{x\}=T \cap X$, then $x \in \mathrm{cl}^{*}(E(M \backslash d)-X)$, which contradicts Lemma 4.3.2.

Let $T$ be a triad that meets $X$ and let $\{c\}=T-X$. The remainder of our arguments in this section are divided into two cases depending on whether the set $X \cup\{c\}$ is fully closed in $M \backslash d$ or not. We begin by analysing the situation when $X \cup\{c\}$ is indeed fully closed.

Lemma 4.3.4. If $X \cup\{c\}$ is fully closed in $M \backslash d$, then $X \cup\{c, d\}$ is either a spike-like 3-separator or a partial spider-like 3-separator with associated partition $\{X,\{c, d\}\}$.

Proof. Assume that $\mathrm{fcl}_{M \backslash d}(X \cup\{c\})=X \cup\{c\}$. By Lemma 4.3.3, each element of $X$ is contained in a triad with $c$ and exactly one other element of $X$. As $X$ does not contain any triad, it follows from this that $|X|$ must be even, and, in particular, that there is a partition of $X$ into pairs $\left\{x_{i}, x_{i}^{\prime}\right\}$ such that $\left\{x_{i}, x_{i}^{\prime}, c\right\}$ is a triad. The element $d$ blocks each of these triads in $M$, and so this partition of $X$ extends to a collection of cocircuits $\left\{x_{i}, x_{i}^{\prime}, c, d\right\}$ of 
$M$. From this partition of $X$ into pairs, we also readily deduce that for all $i \neq j$, the set $\left\{x_{i}, x_{i}^{\prime}, x_{j}, x_{j}^{\prime}\right\}$ is a cocircuit in $M \backslash d$.

4.3.4.1. For each $x \in X,\{x, c, d\}$ is contained in a 4-element circuit $C_{x}$ of $M$.

Proof. $M \backslash d / x$ is 3-connected for all $x \in X$. So, by Bixby's Lemma, $M \backslash d / x, c$ is 3-connected for all $x \in X$ unless $c$ is in a triangle of $M \backslash d / x$. But such a triangle would imply that $X \cup\{c\}$ is not fully closed in $M \backslash d$. Thus $M \backslash d / x, c$ is 3 -connected for all $x \in X$. The absence of a detachable pair in $M$ means that $M / x, c$ cannot be 3 -connected for any $x \in X$, and so $d$ must therefore be in a triangle with every element of $X$ in $M / c$. Thus for all $x \in X,\{x, c, d\}$ is contained in a 4-element circuit $C_{x}$ of $M$.

4.3.4.2. If $\{a, b\}$ is contained in a 4-element cocircuit of $M^{*} / d$ for a $\in$ $\left\{x_{i}, x_{i}^{\prime}\right\}, b \in\left\{x_{j}, x_{j}^{\prime}\right\}$ where $i \neq j$, then this cocircuit is $\left\{x_{i}, x_{i}^{\prime}, x_{j}, x_{j}^{\prime}\right\}$.

Proof. Suppose $a \in\left\{x_{1}, x_{1}^{\prime}\right\}, b \in\left\{x_{2}, x_{2}^{\prime}\right\}$ and that $\{a, b\}$ is contained in a 4-element cocircuit of $M^{*} / d$ which is not $\left\{x_{1}, x_{1}^{\prime}, x_{2}, x_{2}^{\prime}\right\}$. It follows from orthogonality that this cocircuit must contain $c$, and consequently, one of $\left\{x_{3}, x_{3}^{\prime}\right\}$ also. But now we contradict the fact that $c \in \mathrm{cl}_{M^{*} / d}\left(E\left(M^{*} / d\right)-\right.$ $(X \cup\{c\}))$.

4.3.4.3. If $|X|=6$, then $r_{M \backslash d}^{*}(X)=4$.

Proof. Clearly $X=\left\{x_{1}, x_{1}^{\prime}, x_{2}, x_{2}^{\prime}, x_{3}, x_{3}^{\prime}\right\}$ and $r_{M^{*} / d}(X) \in\{3,4\}$. Assume that $r_{M^{*} / d}(X)=3$. If both $\left\{x_{1}, x_{1}^{\prime}, x_{2}, x_{2}^{\prime}\right\}$ and $\left\{x_{2}, x_{2}^{\prime}, x_{3}, x_{3}^{\prime}\right\}$ are cocircuits of $M^{*} / d$, then $E\left(M^{*} / d\right)-X$ must be a flat $M^{*} / d$ of rank at most $r\left(M^{*} / d\right)-$ 2. But then $\lambda_{M^{*} / d}(X)=1$; a contradiction. So we may assume without loss of generality that $\left\{x_{1}, x_{1}^{\prime}, x_{2}, x_{2}^{\prime}\right\}$ is not a cocircuit in $M^{*} / d$. Take any $a \in\left\{x_{1}, x_{1}^{\prime}\right\}, b \in\left\{x_{2}, x_{2}^{\prime}\right\}$. As $r_{M^{*} / d}(X)=3$, it is easily verified that the matroid $M^{*} / d \backslash a, b$ cannot contain a non-trivial 2-separation. By 4.3.4.2, $M^{*} / d \backslash a, b$ also cannot contain a series pair. Thus $M^{*} / d \backslash a, b$ is 3 -connected 
for each $a \in\left\{x_{1}, x_{1}^{\prime}\right\}, b \in\left\{x_{2}, x_{2}^{\prime}\right\}$, and it follows that there exists a 4element cocircuit $C_{a b}$ of $M^{*}$ containing $\{a, b, d\}$ for each such $a, b$. Consider $C_{x_{1} x_{2}}$. This cocircuit meets the circuit $\left\{c, d, x_{3}, x_{3}^{\prime}\right\}$, and so by orthogonality, it must be that $C_{x_{1} x_{2}} \subseteq X \cup\{c, d\}$ with $C_{x_{1} x_{2}} \cap\left\{x_{1}, x_{1}^{\prime}, x_{2}, x_{2}^{\prime}\right\}=\left\{x_{1}, x_{2}\right\}$. Similarly, $C_{x_{1} x_{2}^{\prime}} \subseteq X \cup\{c, d\}$ with $C_{x_{1} x_{2}^{\prime}} \cap\left\{x_{1}, x_{1}^{\prime}, x_{2}, x_{2}^{\prime}\right\}=\left\{x_{1}, x_{2}^{\prime}\right\}$ and $C_{x_{1}^{\prime} x_{2}} \subseteq X \cup\{c, d\}$ with $C_{x_{1}^{\prime} x_{2}} \cap\left\{x_{1}, x_{1}^{\prime}, x_{2}, x_{2}^{\prime}\right\}=\left\{x_{1}^{\prime}, x_{2}\right\}$. Now

$$
E\left(M^{*}\right)-\left(C_{x_{1} x_{2}} \cup C_{x_{1} x_{2}^{\prime}} \cup C_{x_{1}^{\prime} x_{2}}\right)
$$

is a flat of rank at most $r\left(M^{*}\right)-3$, and so

$$
r_{M^{*}}\left(E\left(M^{*}\right)-(X \cup\{c, d\})\right) \leq r\left(M^{*}\right)-3
$$

But then $\lambda(X \cup\{c, d\}) \leq r_{M^{*}}(X \cup\{c, d\})-3=1$, contradicting the fact that $M$ is 3-connected. We conclude that $r_{M \backslash d}^{*}(X) \neq 3$ and so $r_{M \backslash d}^{*}(X)=4$.

4.3.4.4. $\left\{x_{i}, x_{i}^{\prime}, x_{j}, x_{j}^{\prime}\right\}$ is a circuit of $M \backslash d$ for each $i \neq j$.

Proof. If $|X|=4$, then the claim follows immediately. Suppose $|X|=6$ and that $\left\{x_{1}, x_{1}^{\prime}, x_{2}, x_{2}^{\prime}\right\}$ is independent. Then $x_{3} \in \operatorname{cl}_{M \backslash d}\left(\left\{x_{1}, x_{1}^{\prime}, x_{2}, x_{2}^{\prime}\right\}\right)$ because $r_{M \backslash d}(X)=4$, a fact which follows from 4.3.4.3. But this contradicts the fact that $\left\{x_{3}, x_{3}^{\prime}, c\right\}$ is a triad in $M \backslash d$. So the result holds in the case that $|X|=6$ and we may assume that $|X| \geq 8$. Again suppose that $\left\{x_{1}, x_{1}^{\prime}, x_{2}, x_{2}^{\prime}\right\}$ is independent in $M \backslash d$. By 4.3.4.2, we know that for $a \in\left\{x_{1}, x_{1}^{\prime}\right\}, b \in\left\{x_{2}, x_{2}^{\prime}\right\}$, the pair $\{a, b\}$ cannot be contained in a 4-element cocircuit of $M^{*} / d$. Thus the matroid $M^{*} / d \backslash x_{1}$ contains no triads and so by Tutte's Triangle Lemma, either $M^{*} / d \backslash x_{1}, x_{2}$ or $M^{*} / d \backslash x_{1}, x_{2}^{\prime}$ is 3-connected. Assume without loss of generality that $M^{*} / d \backslash x_{1}, x_{2}$ is 3 -connected. As $\left\{x_{1}, x_{2}\right\}$ is not a detachable pair, $M^{*}$ must have a 4 -element cocircuit $\left\{x_{1}, x_{2}, d, \alpha\right\}$ for some $\alpha$. The set $\left\{c, d, x_{3}, x_{3}^{\prime}\right\}$ is a circuit of $M^{*}$, so by orthogonality, $\alpha \in\left\{c, x_{3}, x_{3}^{\prime}\right\}$. The circuit $\left\{c, d, x_{4}, x_{4}^{\prime}\right\}$ then confirms that $\alpha=c$ so that $C_{1}=\left\{c, d, x_{1}, x_{2}\right\}$ is a cocircuit of $M^{*}$. Similarly we obtain another cocircuit $C_{1^{\prime}}$ of $M^{*}$ which must be either $\left\{c, d, x_{1}^{\prime}, x_{2}\right\}$ or $\left\{c, d, x_{1}^{\prime}, x_{2}^{\prime}\right\}$. From the existence of these two 
cocircuits we deduce that $r_{M^{*}}\left(E\left(M^{*}\right)-\left(C_{1} \cup C_{1^{\prime}}\right)\right) \leq r\left(M^{*}\right)-2$. Thus

$$
r_{M^{*} / d}\left(E\left(M^{*} / d\right)-\left\{x_{1}, x_{1}^{\prime}, x_{2}, x_{2}^{\prime}, c\right\}\right) \leq r\left(M^{*} / d\right)-1,
$$

and, as $c \in \mathrm{cl}_{M^{*} / d}\left(E\left(M^{*} / d\right)-\left\{x_{1}, x_{1}^{\prime}, x_{2}, x_{2}^{\prime}\right\}\right)$, this implies that

$$
r_{M^{*} / d}\left(E\left(M^{*} / d\right)-\left\{x_{1}, x_{1}^{\prime}, x_{2}, x_{2}^{\prime}\right\}\right) \leq r\left(M^{*} / d\right)-1 .
$$

As $M^{*} / d$ is 3 -connected, it must now be the case that $\left\{x_{1}, x_{1}^{\prime}, x_{2}, x_{2}^{\prime}\right\}$ is exactly 3 -separating and thus contains a cocircuit of $M^{*} / d$. As $\left\{x_{1}, x_{1}, x_{2}, x_{2}^{\prime}\right\}$ is not itself a cocircuit, this now implies the existence of a triad in $M^{*} / d$, contradicting the fact that $M$ contains no triangles.

4.3.4.5. If $|X| \geq 6$, then $X \cup\{c, d\}$ is a spike-like 3-separator of $M$.

Proof. By 4.3.4.1, $\{x, c, d\}$ is contained in a 4-element circuit of $M$ for each $x \in X$. Suppose that the 4-element circuit of $M$ containing $\left\{x_{1}, c, d\right\}$ has as its fourth element $\alpha \in E(M)-(X \cup\{c, d\})$. Then by orthogonality, $\left\{x_{2}, x_{2}^{\prime}, x_{3}, x_{3}^{\prime}, d\right\}$ cannot be a cocircuit in $M$, so that $\left\{x_{2}, x_{2}^{\prime}, x_{3}, x_{3}^{\prime}\right\}$ must be a cocircuit of $M$. Similarly, $\left\{x_{1}, x_{1}^{\prime}, x_{2}, x_{2}^{\prime}, d\right\}$ is forced to be a cocircuit. We also have a 4 -element circuit containing $\left\{x_{3}, c, d\right\}$, and by orthogonality with these two cocircuits, we deduce, up to labelling, that $\left\{x_{2}, x_{3}, c, d\right\}$ is a circuit of $M$. But now $d \in \operatorname{cl}(X \cup\{c\})$, implying that $\alpha \in \mathrm{cl}_{M \backslash d}(X \cup\{c\})$ when $X \cup\{c\}$ is fully closed in $M \backslash d$. Thus, the four-element circuits containing $\{x, c, d\}$ for each $x \in X$ must be themselves contained in $X$, and it follows that $d \in \operatorname{cl}(X \cup\{c\})$ and $\lambda(X \cup\{c, d\})=2$. Now, considering the matroid $M / c$, we see that $d$ must be in a triangle with every element of $X$, with this triangle being a subset of $X \cup\{d\}$. As $r_{M / c}(X)=\frac{|X|}{2}+1$ and $|X| \geq 6$, it is readily verified that the only allowable configuration of triangles in $M / c$ is where $\left\{d, x_{i}, x_{i}^{\prime}\right\}$ is a triangle for each $i$. Hence $\left\{c, d, x_{i}, x_{i}^{\prime}\right\}$ is a circuit in $M$ for each $i$ and it follows that $\left\{x_{i}, x_{i}^{\prime}, x_{j}, x_{j}^{\prime}\right\}$ is a cocircuit in $M$ for $i \neq j$. We conclude that $X \cup\{c, d\}$ is a spike-like 3-separator.

We now turn to the case where $|X|=4$. Here $X$ is both a circuit and a cocircuit of $M \backslash d$. 
4.3.4.6. If $|X|=4$, then for each $x \in X,\{x, c, d\}$ is contained within a 4-element circuit $C_{x} \subseteq X \cup\{c, d\}$.

Proof. For each $x \in X,\{x, c, d\}$ is contained in a 4-element circuit of $M$ by 4.3.4.1. Suppose that the 4-element circuit $\left\{c, d, x_{1}, \alpha\right\}$ is such that $\alpha \in E(M)-(X \cup\{c, d\})$. Then, as $X \cup\{c\}$ is fully closed in $M \backslash d$, it follows that $d \notin \operatorname{cl}(X \cup\{c\})$ and thus that each of the four circuits $C_{x}$ must meet $E(M)-(X \cup\{c, d\})$. Furthermore, each of these points of intersection must be distinct, and so we have circuits $\left\{c, d, x_{1}, y_{1}\right\},\left\{c, d, x_{1}^{\prime}, y_{1}^{\prime}\right\},\left\{c, d, x_{2}, y_{2}\right\}$ and $\left\{c, d, x_{2}^{\prime}, y_{2}^{\prime}\right\}$, together with a circuit $\left\{y_{1}, y_{1}^{\prime}, y_{2}, y_{2}^{\prime}\right\}$. Suppose now that $M \backslash x_{1}, y_{1}^{\prime}$ does not contain a series pair. Then by Lemma 1.2.6, it has a 2-separation $(F, G)$ with the triad $\left\{x_{1}^{\prime}, c, d\right\} \subseteq F$. But then $y_{1}^{\prime} \in \operatorname{cl}_{M \backslash x_{1}}(F)$ giving a 2-separation $\left(F \cup\left\{y_{1}^{\prime}\right\}, G\right)$ in the 3-connected matroid $M \backslash x_{1}$. Thus $M \backslash x_{1}, y_{1}^{\prime}$ must contain a series pair which extends to a 4-element cocircuit of $M$. By orthogonality with the circuits $\left\{x_{1}, x_{1}^{\prime}, x_{2}, x_{2}^{\prime}\right\},\left\{y_{1}, y_{1}^{\prime}, y_{2}, y_{2}^{\prime}\right\},\left\{c, d, x_{1}, y_{1}\right\}$ and $\left\{c, d, x_{1}^{\prime}, y_{1}^{\prime}\right\}$, we observe that the only allowable such cocircuit is $C_{1}=\left\{x_{1}, x_{1}^{\prime}, y_{1}, y_{1}^{\prime}\right\}$. By an identical argument, we also uncover the cocircuit $C_{2}=\left\{x_{2}, x_{2}^{\prime}, y_{2}, y_{2}^{\prime}\right\}$. Consider now the matroid $M \backslash x_{1}, y_{2}$. If $C_{3}=\left\{x_{1}, y_{1}, x_{2}, y_{2}\right\}$ is a cocircuit of $M$, then

$$
\begin{array}{r}
E(M)-\left(C_{1} \cup C_{2} \cup C_{3} \cup\left\{c, d, x_{1}, x_{1}^{\prime}\right\}\right) \\
=E(M)-(X \cup\{c, d\})
\end{array}
$$

is a flat of rank at most $r(M)-4$, implying that it must be 2-separating. So $\left\{x_{1}, y_{1}, x_{2}, y_{2}\right\}$ cannot be a cocircuit, and it follows that $M \backslash x_{1}, y_{2}$ does not contain a series pair. By Lemma 1.2.6, $M \backslash x_{1}, y_{2}$ has a 2-separation $(P, Q)$ in which $\left\{c, d, x_{1}^{\prime}\right\} \subseteq P$. Freely applying Lemma 1.2.6, we may assume that $y_{1}^{\prime} \in P$ and $y_{1} \in P$ by virtue of the circuit $\left\{c, d, x_{1}^{\prime}, y_{1}^{\prime}\right\}$ and the triad $\left\{y_{1}, y_{1}^{\prime}, x_{1}^{\prime}\right\}$. Certainly $y_{2}^{\prime} \in Q$, as otherwise $\left(P \cup\left\{y_{2}\right\}, Q\right)$ would be a 2-separation of $M \backslash x_{1}$, and it must be that $x_{2} \in Q$ for the same reason. Taking coclosure, we may then assume that the triad $\left\{x_{2}, x_{2}^{\prime}, y_{2}^{\prime}\right\}$ is contained in $Q$. Let $Y=\left\{y_{1}, y_{1}^{\prime}, y_{2}, y_{2}^{\prime}\right\}$. Now if $r_{M \backslash x_{1}, y_{2}}\left(\left\{c, d, x_{1}^{\prime}, y_{1}, y_{1}^{\prime}\right\}\right)=3$, then 
$\left\{y_{1}, y_{1}^{\prime}, x_{1}^{\prime}, d\right\}$ is a circuit which meets the cocircuit $\left\{c, d, x_{2}, x_{2}^{\prime}\right\}$ at exactly one point; a contradiction. Thus

$$
\begin{array}{r}
r_{M \backslash x_{1}, y_{2}}(P \cap(X \cup Y \cup\{c, d\}))=4 \\
\text { and } r_{M \backslash x_{1}, y_{2}}(Q \cap(X \cup Y \cup\{c, d\}))=3 .
\end{array}
$$

By Lemma 1.2.5, $(P \cap(X \cup Y \cup\{c, d\}), Q \cap(X \cup Y \cup\{c, d\}))$ is 2-separating in $\left(M \backslash x_{1}, y_{2}\right) \mid(X \cup Y \cup\{c, d\})$. But as we have seen, in this restriction,

$$
\begin{array}{r}
r_{\left(M \backslash x_{1}, y_{2}\right) \mid(X \cup Y \cup\{c, d\})}(P)+r_{\left(M \backslash x_{1}, y_{2}\right) \mid(X \cup Y \cup\{c, d\})}(Q) \\
-r_{\left(M \backslash x_{1}, y_{2}\right) \mid(X \cup Y \cup\{c, d\})}(P \cup Q) \\
=\lambda_{\left(M \backslash x_{1}, y_{2}\right) \mid(X \cup Y \cup\{c, d\})}(P)=2,
\end{array}
$$

and we arrive at a contradiction.

4.3.4.7. If $|X|=4$, then each element of $\left\{x_{1}, x_{1}^{\prime}\right\}$ is contained in a 4-element cocircuit $C_{x}$ of $M$ with $C_{x} \subseteq X \cup\{c, d\}$ and $C_{x} \cap\left\{x_{2}, x_{2}^{\prime}\right\} \neq \emptyset$.

Proof. Assume that no series pair exists within $\left\{c, d, x_{1}^{\prime}, x_{2}^{\prime}\right\}$ in the matroid $M \backslash x_{1}, x_{2}$. As $X \cup\{c\}$ is fully closed in $M \backslash d$, it follows from 4.3.4.6 and Lemma 1.2.7 that $X \cup\{c, d\}$ is fully closed in $M$. Thus, if $M \backslash x_{1}, x_{2}$ contains some series pair which meets $E(M)-(X \cup\{c, d\})$, then this series pair must be fully contained in $E(M)-(X \cup\{c, d\})$. So either $M$ has a 4-element cocircuit whose intersection with $X \cup\{c, d\}$ is $\left\{x_{1}, x_{2}\right\}$ or $M \backslash x_{1}, x_{2}$ has a non-trivial 2-separation $(P, Q)$. In the former case, $x_{1} \in \mathrm{cl}^{*}\left(E(M)-\left\{c, d, x_{1}^{\prime}, x_{2}^{\prime}\right\}\right)$, implying that $x_{1} \notin \operatorname{cl}\left(\left\{c, d, x_{1}^{\prime}, x_{2}^{\prime}\right\}\right)$, so that $\left\{c, d, x_{1}^{\prime}, x_{2}^{\prime}\right\}$ must be a circuit. In the latter case, as $\left\{c, d, x_{1}^{\prime}\right\}$ is a triad of $M \backslash x_{1}, x_{2}$, we may assume by Lemma 1.2.6 that $\left\{c, d, x_{1}^{\prime}\right\} \subseteq P$. If $x_{1}$ or $x_{2}$ is in $\operatorname{cl}(P)$, we now obtain a contradictory 2-separation in one of the 3-connected matroids $M \backslash x_{2}$ or $M \backslash x_{1}$. By 4.3.4.6 we conclude that, as in the previous case, $\left\{c, d, x_{1}^{\prime}, x_{2}^{\prime}\right\}$ must be a circuit of $M$. Suppose now that $M \backslash x_{1}, x_{2}^{\prime}$ does not contain a series pair within $\left\{c, d, x_{1}^{\prime}, x_{2}\right\}$. If $\left(P^{\prime}, Q^{\prime}\right)$ is a non-trivial 2-separation of $M \backslash x_{1}, x_{2}^{\prime}$, we may as before assume by Lemma 1.2.6 that $\left\{c, d, x_{1}^{\prime}\right\} \subseteq P^{\prime}$. But $\left\{c, d, x_{1}^{\prime}, x_{2}^{\prime}\right\}$ is a circuit in $M \backslash x_{1}$, implying that $\left(P^{\prime} \cup\left\{x_{2}^{\prime}\right\}, Q\right)$ is a 
2-separation of the 3-connected matroid $M \backslash x_{1}$. Hence $\operatorname{co}\left(M \backslash x_{1}, x_{2}^{\prime}\right)$ must be 3-connected and $M$ must have a 4-element cocircuit whose intersection with $X \cup\{c, d\}$ is $\left\{x_{1}, x_{2}^{\prime}\right\}$. As argued above, this shows that $\left\{c, d, x_{1}^{\prime}, x_{2}\right\}$ is a circuit in $M$. But both $\left\{c, d, x_{1}^{\prime}, x_{2}^{\prime}\right\}$ and $X$ are also circuits and so we contradict the fact that $r(X \cup\{c, d\})=4$. We can conclude that at least one of $M \backslash x_{1}, x_{2}$ and $M \backslash x_{1}, x_{2}^{\prime}$ must contain a series pair which is contained in $\left\{c, d, x_{1}^{\prime}, x_{2}, x_{2}^{\prime}\right\}$. Thus $x_{1}$ is in some 4-element cocircuit $C_{x}$ of $M$ with $C_{x} \cap\left\{x_{2}, x_{2}^{\prime}\right\} \neq \emptyset$ and $C_{x} \subseteq X \cup\{c, d\}$. The same argument shows that this also holds for $x_{1}^{\prime}$.

Suppose that the 4-element cocircuit $C_{x_{1}}$ containing $x_{1}$ obtained from 4.3.4.7 is such that $\left|C_{x_{1}} \cap X\right|=3$. Then, as both $\left\{c, d, x_{1}, x_{1}^{\prime}\right\}$ and $\left\{c, d, x_{2}, x_{2}^{\prime}\right\}$ are cocircuits of $M$, we have that $X \cup\{c, d\} \subseteq \operatorname{cl}^{*}\left(C_{x_{1}}\right)$. This contradicts the fact that $r^{*}(X \cup\{c, d\})=4$. Suppose next that there exists cocircuits $C_{x_{1}}=\left\{c, d, x_{1}, \alpha\right\}$ and $C_{x_{1}^{\prime}}=\left\{c, d, x_{1}^{\prime}, \beta\right\}$ where $\alpha, \beta \in\left\{x_{2}, x_{2}^{\prime}\right\}$. Then $\alpha \neq \beta$ as $r^{*}(X \cup\{c, d\}) \neq$,3 . But now

$$
\begin{array}{r}
E(M)-\left(\left\{c, d, x_{1}, x_{1}^{\prime}\right\} \cup C_{x_{1}} \cup C_{x_{1}^{\prime}}\right) \\
=E(M)-(X \cup\{c, d\})
\end{array}
$$

is a flat of rank at most $r(M)-3$, which contradicts the fact that $X \cup\{c, d\}$ is exactly 3 -separating in $M$. The only remaining possibility is that $X$ itself is a cocircuit in $M$, and in particular, that $X$ is the unique cocircuit of $M$ which meets both of $\left\{x_{1}, x_{1}^{\prime}\right\}$ and $\left\{x_{2}, x_{2}^{\prime}\right\}$. Recall from 4.3.4.1 that for each $x \in X$, there exists a 4-element circuit $\{c, d, x, \alpha\}$ of $M$ where $\alpha \in X$. If $\left\{c, d, x_{1}, x_{1}^{\prime}\right\}$ is a circuit, then $\left\{c, d, x_{2}, x_{2}^{\prime}\right\}$ must also be a circuit because $r(X \cup\{c, d\})=$, 4. In this situation $X \cup\{c, d\}$ is a spike-like 3-separator. Otherwise $X \cup\{c, d\}$ is a partial spider-like 3 -separator with associated partition $\{X,\{c, d\}\}$.

With the completion of Lemma 4.3.4, we now turn our attention to the case where $X \cup\{c\}$ fails to be fully closed in $M \backslash d$. 
Lemma 4.3.5. If $X \cup\{c\}$ is not fully closed in $M \backslash d$, then there exists a set $Y \subseteq E(M)-X$ with $d \in Y$ such that $X \cup Y$ is a spider-like 3-separator with associated partition $\{X, Y\}$.

Proof. Assume that $X \cup\{c\}$ is not fully closed in $M \backslash d$. Then there exists a sequence $\left(s_{1}, \ldots, s_{n}\right)$ such that $X \cup\left\{c, s_{1}, \ldots, s_{n}\right\}$ is a fully closed proper 3-separating subset of $M \backslash d$ where

$$
s_{n} \in \mathrm{cl}^{(*)}\left(X \cup\left\{c, s_{1}, \ldots, s_{n-1}\right\}\right) \cap \mathrm{cl}^{(*)}\left(E(M \backslash d)-\left(X \cup\left\{s_{1}, \ldots, s_{n}\right\}\right)\right) .
$$

If $s_{n} \in \operatorname{cl}\left(X \cup\left\{c, s_{1}, \ldots, s_{n-1}\right\}\right)$, then, as $M \backslash d, s_{n}$ cannot be 3-connected, $s_{n}$ must be contained in some triad of $M \backslash d$. But such a triad must intersect both $X \cup\left\{c, s_{1}, \ldots, c_{n-1}\right\}$ and $E(M)-\left(X \cup\left\{c, s_{1}, \ldots, c_{n}\right\}\right)$, which contradicts the fact that $X \cup\left\{c, s_{1}, \ldots, s_{n}\right\}$ is fully closed in $M \backslash d$. So

$$
s_{n} \in \mathrm{cl}^{*}\left(X \cup\left\{c, s_{1}, \ldots, s_{n-1}\right\}\right) \cap \mathrm{cl}^{*}\left(E(M \backslash d)-\left(X \cup\left\{c, s_{1}, \ldots, s_{n}\right\}\right)\right) .
$$

Let $c^{\prime}=s_{n}$.

4.3.5.1. $M \backslash d / c, c^{\prime}$ is 3-connected, as is $M \backslash d / x, c^{\prime}$ for all $x \in X$.

Proof. The absence of triangles in $\mathrm{fcl}_{M}(X \cup\{d\})$ implies the absence of triangles in $\mathrm{fcl}_{M \backslash d}(X)$, and so by Bixby's Lemma, $M \backslash d / c$ is 3-connected. If $M \backslash d / c, c^{\prime}$ fails to be 3 -connected, there must be some 4-element circuit $C$ of $M \backslash d$ with $\left\{c, c^{\prime}\right\} \subseteq C$. As $c$ is in a triad with two elements from $X$ in $M \backslash d$, we must have $C \cap X \neq \emptyset$. One possibility for the location of the fourth element of $C$ is within $X \cup\left\{s_{1}, \ldots, s_{n-1}\right\}$, but this contradicts the fact that

$$
c^{\prime} \in \mathrm{cl}^{*}\left(E(M \backslash d)-\left(X \cup\left\{c, s_{1}, \ldots, c^{\prime}\right\}\right)\right) .
$$

Therefore $C$ must meet $E(M \backslash d)-\left(X \cup\left\{c, s_{1}, \ldots, c^{\prime}\right\}\right)$, contradicting the fact that $X \cup\left\{c, s_{1}, \ldots, c^{\prime}\right\}$ is fully closed in $M \backslash d$. The proof that $M \backslash d / x, c^{\prime}$ is 3-connected for all $x \in X$ proceeds similarly. 
As $M \backslash d / c, c^{\prime}$ is 3-connected and $\operatorname{fcl}(X \cup\{d\})$ does not contain a detachable pair, $M / c^{\prime}$ must contain a triangle $\{c, d, \alpha\}$. Similarly, every element of $x$ must be in a triangle with $d$ in $M / c^{\prime}$. Suppose to begin with that $\alpha \in X$. Note that for all $x \in X-\{\alpha\}$, it must be the case that $x \notin \operatorname{cl}(\{c, d, \alpha\})$. This is because the existence of a 4-point line in $M / c^{\prime}$ would, as $\operatorname{cl}_{M \backslash d / c^{\prime}}(X \cup\{c\})=\operatorname{cl}_{M \backslash d}(X \cup\{c\})$, imply the existence of a 4point line in $M$, giving a contradictory triangle in $M \backslash d$. Let $x_{1}, x_{2}, x_{3}$ be distinct elements of $X-\{\alpha\}$. Then there are triangles $\left\{d, x_{1}, g_{1}\right\},\left\{d, x_{2}, g_{2}\right\}$ and $\left\{d, x_{3}, g_{3}\right\}$ in $M / c^{\prime}$. If $d \in \operatorname{cl}_{M / c^{\prime}}(X)$, then $c \in \mathrm{cl}_{M / c^{\prime}}(X)$, contradicting the fact that $c \in \mathrm{cl}_{M \backslash d / c^{\prime}}^{*}\left(E\left(M \backslash d / c^{\prime}\right)-X\right)$. So $d \notin \mathrm{cl}_{M / c^{\prime}}(X)$ and the elements $g_{1}, g_{2}, g_{3}$ must be distinct with

$$
\left\{g_{1}, g_{2}, g_{3}\right\} \subseteq\left(\mathrm{cl}_{M / c^{\prime}}(X \cup\{c\})-(X \cup\{c, d\})\right)
$$

But now $\left\{g_{1}, g_{2}, g_{3}\right\}$ is a triangle in $M / c^{\prime}$, and therefore, as $\operatorname{cl}_{M \backslash d / c^{\prime}}(X \cup\{c\})=$ $\operatorname{cl}_{M \backslash d}(X \cup\{c\})$, a triangle in $M$. We may now assume that $\alpha \notin X$. Here $d \in \mathrm{cl}_{M / c^{\prime}}\left(E\left(M / c^{\prime}\right)-X\right)$.

4.3.5.2. For each $x \in X,\{d, x\}$ is contained in a triangle $T_{x}$ of $M / c^{\prime}$ where $\left|T_{x} \cap X\right|=2$.

Proof. Every element of $X$ is certainly in a triangle with $d$ in $M / c^{\prime}$ by 4.3.5.1. By 4.3.2, $X \cap\left(\operatorname{cl}_{M \backslash d / c^{\prime}}\left(E\left(M \backslash d / c^{\prime}\right)-X\right)\right)=\emptyset$. As $d \in \mathrm{cl}_{M / c^{\prime}}\left(E\left(M / c^{\prime}\right)-X\right)$, it follows that $X \cap \mathrm{cl}_{M / c^{\prime}}\left(E\left(M / c^{\prime}\right)-X\right)=\emptyset$, and thus the triangles containing $d$ and $x$ for each $x \in X$ must have two points in $X$.

From 4.3.5.2 it follows that $\alpha \in \mathrm{cl}_{M \backslash d / c^{\prime}}(X \cup\{c\})$, and as

$$
\left(M \backslash d / c^{\prime}\right)\left|\left(X \cup\left\{c, s_{1}, \ldots, s_{n-1}\right\}\right)=(M \backslash d)\right|\left(X \cup\left\{c, s_{1}, \ldots, s_{n-1}\right\}\right),
$$

we have that $\alpha \in \mathrm{cl}_{M \backslash d}(X \cup\{c\})$. We may thus assume that $\alpha=s_{1}$.

4.3.5.3. $\left\{s_{1}, \ldots, s_{n}\right\}=\left\{\alpha, c^{\prime}\right\}$. 
Proof. Suppose that $n>2$. Then $\operatorname{si}\left(M / c^{\prime}, s_{2}\right)$ is 3 -connected, so $s_{2}$ must be in a triangle $T^{\prime}$ of $M / c^{\prime}$. Now $T^{\prime}$ must meet $E(M)-\left(X \cup\left\{c, d, \alpha, s_{2}\right\}\right)$ so that $T^{\prime}$ is a triangle of $M \backslash d$ unless $T^{\prime} \cap(X \cup\{d, c, \alpha\})=\{d\}$. But then $d \notin \mathrm{cl}_{M}^{*}(X \cup\{c\})$ implying that $T$ is a contradictory triad in $M$.

4.3.5.4. $\left\{c, \alpha, c^{\prime}, d\right\}$ is a circuit and a cocircuit of $M$.

Proof. The set $\left\{c, \alpha, c^{\prime}\right\}$ is a triad in $M \backslash d$, for otherwise, $M \backslash d, \alpha$ is 3connected. The element $d$ must block this triad in $M$ and so $\left\{c, \alpha, c^{\prime}, d\right\}$ is a cocircuit of $M$. As $\{c, d, \alpha\}$ is a triangle of $M / c^{\prime}$, the set $\left\{c, \alpha, c^{\prime}, d\right\}$ is also a circuit of $M$.

Note that

$$
\begin{gathered}
\alpha \in \operatorname{cl}_{M \backslash d}(E(M \backslash d)-(X \cup\{c, \alpha\})) \\
-\operatorname{cl}_{M \backslash d}\left(E(M \backslash d)-\left(X \cup\left\{c, \alpha, c^{\prime}\right\}\right)\right),
\end{gathered}
$$

so that $c^{\prime} \in \mathrm{cl}_{M \backslash d}\left(E(M \backslash d)-\left(X \cup\left\{c, c^{\prime}\right\}\right)\right.$. Therefore each of the triads of $M \backslash d$ obtained from 4.3.3 must contain $c$. Consider such a triad $\Gamma$. By 4.3.5.2, there exists a number of triangles in $M / c^{\prime}$; each of which contains $d$ as well as two elements from $X$. Suppose that $T^{\prime}$ is such a triangle where $T^{\prime} \cap X \cap \Gamma=\emptyset$. Then $\left(T^{\prime} \cap X\right) \cup\{c, \alpha\}$ is a 4-element circuit of $M$ whose intersection with the cocircuit $\Gamma \cup\{d\}$ is $\{c\}$; a contradiction by orthogonality. This now implies that $X$ is both a circuit and a cocircuit with $|X|=4$. In particular, labelling $X$ as $\left\{x_{1}, x_{2}, x_{3}, x_{4}\right\}$ where $\left\{x_{1}, x_{2}, d\right\}$ and $\left\{x_{3}, x_{4}, d\right\}$ are triangles of $M / c^{\prime}$, we have that up to our choice of labelling, $\left\{x_{1}, x_{3}, c, d\right\}$ and $\left\{x_{2}, x_{4}, c, d\right\}$ are cocircuits of $M$. Now

$$
E(M)-\left(\left\{x_{1}, x_{3}, c, d\right\} \cup\left\{x_{1}, x_{2}, x_{3}, x_{4}\right\} \cup\left\{c, d, c^{\prime}, \alpha\right\}\right)
$$

is a flat of rank at most $r(M)-3$. This implies that $r\left(X \cup\left\{c, d, c^{\prime}, \alpha\right\}\right)=5$ as $X \cup\left\{c, d, c^{\prime}, \alpha\right\}$ is exactly 3 -separating, and therefore that $\sqcap\left(X,\left\{c, d, c^{\prime}, \alpha\right\}\right)=$ 1. Consider $M / \alpha$. Certainly this matroid is 3 -connected as $\left\{c, d, c^{\prime}, \alpha\right\}$ is a 
closed quad. Now the set $X$ is a quad of $M / \alpha$ and so $\operatorname{si}(M / \alpha, x)$ is 3 connected for all $x \in X$. If we are not to have a detachable pair, every element of $X$ must be in a triangle of $M / \alpha$ and thus in a 4-element circuit with $\alpha$ in $M$. Checking orthogonality with the cocircuits $\left\{x_{1}, x_{3}, c, d\right\}$ and $\left\{x_{2}, x_{4}, c, d\right\}$, the only possibility is that $\left\{x_{1}, x_{2}, c, \alpha\right\}$ and $\left\{x_{3}, x_{4}, c, \alpha\right\}$ are the circuits in question. All that remains now is to show that $\left\{x_{1}, x_{3}, c^{\prime}, \alpha\right\}$ and $\left\{x_{2}, x_{4}, c^{\prime}, \alpha\right\}$ are cocircuits. The matroid $M / \alpha$ is 3 -connected and $\operatorname{co}(M / \alpha, x)$ is 3-connected by virtue of the fact that $X$ is a quad in $M / \alpha$. Thus $\{\alpha, x\}$ is contained in a 4-element cocircuit $C_{x}$ for each $x \in X$. These cocircuits have two elements in $X$ and two in $\left\{c, d, c^{\prime}, \alpha\right\}$ by orthogonality. Suppose that for some $x \in X$, $c^{\prime} \notin C_{x}$. Then

$$
E(M)-\left(C_{x} \cup\left\{x_{1}, x_{3}, c, d\right\} \cup\left\{x_{1}, x_{2}, x_{3}, x_{4}\right\} \cup\left\{c, d, c^{\prime}, \alpha\right\}\right)
$$

must be a flat of rank at most $r(M)-4$. This contradicts the previously established fact that $r\left(E(M)-\left(X \cup\left\{c, d, c^{\prime}, \alpha\right\}\right)\right)=r(M)-3$. So $c^{\prime} \in C_{x}$ for each $x \in X$. A virtually identical argument shows that we must have $x_{3} \in C_{x_{1}}$ and $x_{4} \in C_{x_{2}}$. Now $X \cup\left\{c, d, c^{\prime}, \alpha\right\}$ is now a spider-like 3-separator with associated partition $\left(X,\left\{c, d, c^{\prime}, \alpha\right\}\right)$ as required.

With that, the proof of Theorem 4.3.1 is complete. 


\section{Chapter 5}

\section{Putting the Pieces Together}

The groundwork that has been laid down in Chapter 3 and Chapter 4 will be brought together into a much more digestible form in Theorem 5.2.1. From that point, our main result, Theorem 1.0.1, is a straightforward extension. Before arriving at that stage however, we still need to prove a few more preparatory lemmas.

\subsection{Further Lemmas}

Lemma 5.1.1. Let $M$ be a 3-connected matroid which contains no triangles or triads. If $M$ contains a vertical 3-separation, then there exists a vertical 3-separation $(P,\{r\}, Q)$ of either $M$ or $M^{*}$ where $P \cup\{r\}$ is fully closed.

Proof. Suppose that $(X,\{e\}, Y)$ is a vertical 3-separation of $M$ where $X \cup\{e\}$ is not fully closed. As $M$ does not contain any triangles or triads, $M$ is certainly non-sequential. As such, we may assume that $Y$ has no sequential ordering. Therefore there must exist some sequence $\left(y_{1}, \ldots, y_{n}\right)$ of elements of $Y$ such that $X \cup\left\{e, y_{1}, \ldots, y_{n}\right\}$ is fully closed. Setting $P$ as $X \cup\left\{e, y_{1}, \ldots, y_{n-1}\right\}$, setting $r$ as $y_{n}$, and setting $Q$ as $Y-\left\{y_{1}, \ldots, y_{n}\right\}$, we note that both $P$ and $Q$ have rank three or more and each must contain circuits 
by virtue of the fact that $Y$ does not have a sequential ordering. Therefore $(P,\{r\}, Q)$ is either a vertical 3-separation of $M$ or a cyclic 3-separation of $M$, according to whether $r \in \operatorname{cl}(P)$ or $r \in \mathrm{cl}^{*}(P)$ respectively.

Lemma 5.1.2. Let $M$ be a 3-connected matroid which contains no triangles or triads where $|E(M)|>9$. If $M$ contains a vertical 3-separation, then $M$ contains a detachable pair.

Proof. Suppose that $M$ has some vertical 3-separation. By Lemma 5.1.1, there exists a vertical 3-separation $(P,\{r\}, Q)$ of either $M$ or $M^{*}$ where $P \cup$ $\{r\}$ is fully closed. As shall become clear, the distinction is irrelevant for our purposes, but for the sake of completeness, let $M^{\prime}$ be the element of $\left\{M, M^{*}\right\}$ for which $(P,\{r\}, Q)$ is vertical. Certainly $M^{\prime} \backslash r$ is 3-connected by Bixby's Lemma and the fact that $M^{\prime}$ contains no triads. By Lemma 1.2.8, $P$ is fully closed in $M^{\prime} \backslash r$. It follows from this observation that $P$ cannot contain any triads of $M^{\prime} \backslash r$, as $r$ would need to block any such triad in $M^{\prime}$; giving the contradiction that $r \in \mathrm{cl}_{M^{\prime}}^{*}(P)$ when we know that $r \in \mathrm{cl}_{M^{\prime}}(Q)$. Applying Lemma 1.2.23 to the 3-separation $(P, Q)$, we conclude that there exists some $p \in P$ such that $M^{\prime} \backslash r, p$ is 3-connected, giving a detachable pair in $M$.

Lemma 5.1.3. Let $M$ be a 3-connected matroid with $|E(M)|>9$ such that $M$ contains no triangles or triads. Then either $M$ contains a detachable pair, or, for all $e \in E(M)$, both $M \backslash e$ and $M /$ e are 3 -connected.

Proof. By Lemma 5.1.2 together with its dual, if $M$ does not have a detachable pair, then $M$ cannot contain any vertical or cyclic 3-separations. It now follows from Lemma 1.2.11 together with the fact that $M$ contains no triangles or triads that both $M \backslash e$ and $M / e$ must be 3-connected for every $e \in E(M)$.

Lemma 5.1.4. Let $M$ be a 3-connected matroid with the property that both $M \backslash e$ and $M / e$ are 3-connected for every $e \in E(M)$. Let $(X, Y)$ be an exact 3-separation of $M$ where both $X$ and $Y$ are fully closed. Then either $M$ contains a detachable pair, or else for every $x \in X$ and every $y \in Y$, there 
exists a 4-element circuit $C$ in $M$ with $\{x, y\} \subseteq C$, as well as a 4-element cocircuit $C^{*}$ in $M$ with $\{x, y\} \subseteq C^{*}$.

Proof. Assume that $M$ contains no detachable pair and take any $x \in X$, $y \in Y$.

5.1.4.1. If $Z \subseteq X-\{x\}$ or $Z \subseteq Y-\{y\}$, and $|Z| \geq 2$, then $\lambda_{M / x, y}(Z) \neq$ $1 \neq \lambda_{M \backslash x, y}(Z)$.

Proof. Suppose that $Z \subseteq X-\{x\}$ where $|Z| \geq 2$ and $\lambda_{M \backslash x, y}(Z)=1$. We know that the matroid $M \backslash x$ is 3-connected. Therefore, as $|Z| \geq 2$, it must be that $Z$ is exactly 3 -separating in $M \backslash x$. As $\lambda_{M \backslash x}(Z)=r_{M \backslash x}(Z)+r_{M \backslash x}^{*}(Z)-$ $|Z|=2$, this now implies that $y \in \operatorname{cl}_{M \backslash x}^{*}(Z)$. However, $Z \subseteq X-\{x\}$ and $X-\{x\}$ is fully closed in $M \backslash x$ by Lemma 1.2 .8 , and so we arrive at a contradiction. Thus, whenever $Z \subseteq X-\{x\}$ and $|Z| \geq 2$ it must be that $\lambda_{M \backslash x, y}(Z) \neq 1$, and, similarly, $\lambda_{M / x, y}(Z) \neq 1$. Essentially the same argument shows that this also holds for any $Z \subseteq Y-\{y\}$.

Let $(P, Q)$ be a 2-separation of $M / x, y$. By 5.1.4.1, $(P, Q)$ must cross $(X-\{x\}, Y-\{y\})$, giving four non-empty intersections $P \cap(X-\{x\}), P \cap$ $(Y-\{y\}), Q \cap(X-\{x\})$ and $Q \cap(Y-\{y\})$. As both $X$ and $Y$ are fully closed in $M$, we certainly know that both $|X-\{x\}|$ and $|Y-\{y\}|$ exceed two. Therefore we may assume that $|P \cap(X-\{x\})| \geq 2$. By submodularity of the connectivity function, together with the fact, which follows from 5.1.4.1, that $\lambda_{M / x, y}(X-\{x\})=2$, we have

$$
\begin{array}{r}
\lambda_{M / x, y}(P \cap(X-\{x\}))+\lambda_{M / x, y}(P \cup(X-\{x\})) \\
\leq \lambda_{M / x, y}(P)+\lambda_{M / x, y}(X-\{x\}) .
\end{array}
$$

Thus

$$
\lambda_{M / x, y}(P \cap(X-\{x\}))+\lambda_{M / x, y}(P \cup(X-\{x\})) \leq 3,
$$

so that

$$
\lambda_{M / x, y}(P \cap(X-\{x\}))+\lambda_{M / x, y}(Q \cap(Y-\{y\})) \leq 3
$$


Therefore at least one of $P \cap(X-\{x\})$ and $Q \cap(Y-\{y\})$ must be 2separating in $M / x, y$. By 5.1.4.1, $P \cap(X-\{x\})$ cannot be 2-separating, and so $Q \cap(Y-\{y\})$ must be. Thus, again using 5.1.4.1, we see that $Q \cap(Y-\{y\})$ must be a singleton. Consequently, $|P \cap(Y-\{y\})| \geq 2$, and repeating the same submodularity argument as above, we are forced into a situation where at least one of $P \cap(Y-\{y\})$ and $Q \cap(X-\{x\})$ is 2-separating in $M / x, y$. Once again, the only possibility that does not contradict 5.1.4.1 is that $|Q \cap(X-\{x\})|=1$. Thus $|Q|=2$ so that $Q$ is a parallel pair of $M / x, y$. The matroid $M$ obviously does not contain any triangles and so $Q$ must be lifted in such a way that $Q \cup\{x, y\}$ is a 4-element circuit of the matroid $M$ as required. The fact that $\{x, y\}$ is also contained in a 4-element cocircuit of $M$ follows in the same manner simply by examining the matroid $M \backslash x, y$ as opposed to $M / x, y$.

\subsection{Proof of Main Theorem}

We approach a proof of Theorem 1.0.1.

Theorem 5.2.1. Let $M$ be a 3-connected matroid which contains no triangles or triads where $|E(M)|>10$. If $M$ does not contain a detachable pair, then $M$ contains a fully closed exactly 3-separating set $X$ such that either:

(i) $X$ is a twisted 3-separator,

(ii) $X$ is a partial spider-like 3 -separator,

(iii) $X$ is a spider-like 3-separator, or

(iv) $X$ is a spike-like 3-separator.

Proof. Assume that $M$ does not contain a detachable pair, and let $d$ be any element of $E(M)$. By Lemma 5.1.3, the matroid $M \backslash d$ is 3-connected. We know from Lemma 3.1.1 that $M \backslash d$ cannot be sequential, and, furthermore, 
from Lemma 3.2.1 that $M \backslash d$ is not internally 4-connected. Thus there must exist some 3 -separation $(P, Q)$ of $M \backslash d$ such that $|P| \geq 4$ and $|Q| \geq 4$ where $P$ has no possible sequential ordering. Consider now the 3 -separating set $Q$ and apply Lemma 4.1.1. We deduce the existence of a 3-separating set $Q^{\prime} \subseteq Q$ of $M \backslash d$ with the properties that $\left|Q^{\prime}\right| \geq 4$, and that the matroids $M \backslash d / q^{\prime}$ and $\operatorname{co}\left(M \backslash d, q^{\prime}\right)$ are 3-connected for all $q^{\prime} \in Q^{\prime}$. The set $Q^{\prime}$ either contains a triad of $M \backslash d$ or it does not. In the former case, Theorem 4.2.1 shows that (i), (ii) or (iv) must hold. In the latter case, Theorem 4.3.1 implies that (ii), (iii) or (iv) holds. With that we conclude the proof of the theorem.

Lemma 5.2.2. Let $M$ be a 3-connected matroid which contains no triangles or triads where $|E(M)|>12$. If $M$ does not contain a detachable pair, then $M$ is a spike.

Proof. Assume that $M$ does not contain any detachable pair. By Theorem 5.2.1, there exists a fully closed exactly 3 -separating set $X$ which is one of four possible types given by outcomes (i)-(iv). Note that in each of the four cases, every element from $X$ is contained in a four-element circuit $C$ with $C \subseteq X$ as well as a four-element cocircuit $C^{*}$ with $C^{*} \subseteq X$. It follows from this that both $X$ and $Y=E(M)-X$ are fully closed in $M$. Equipped with this fact, and combining it with Lemma 5.1.3, we are now free to use Lemma 5.1.4 to put necessary constraints on the configuration of points in $Y$.

Suppose to begin with that 5.2.1 (ii) holds so that $X$ is a partial spiderlike 3-separator. Let $\left\{\left\{p_{1}, p_{2}, p_{3}, p_{4}\right\},\left\{q_{1}, q_{2}\right\}\right\}$ be the associated partition of $X$. Let $y_{1}$ be any element of $Y$. By Lemma 5.1.4, there must exist some 4-element circuit $C_{1}$ which contains both $y_{1}$ and $q_{1}$. As both $\left\{q_{1}, q_{2}, p_{1}, p_{3}\right\}$ and $\left\{q_{1}, q_{2}, p_{2}, p_{4}\right\}$ are cocircuits of $M$, it follows from orthogonality and the fact that $X$ is closed that $C_{1} \cap X=\left\{q_{1}, q_{2}\right\}$. Let $\left\{y_{2}\right\}=C_{1} \cap\left(Y-\left\{y_{1}\right\}\right)$. Now again apply Lemma 5.1.4, this time with respect to the points $y_{1}$ and $p_{1}$. Using the same argument, we find that there exists some $y_{3} \in Y-\left\{y_{1}, y_{2}\right\}$ such that $C_{2}=\left\{y_{1}, y_{3}, p_{1}, p_{3}\right\}$ is a circuit. Repeating this process a third time 
then gives the existence of a $y_{4} \in Y-\left\{y_{1}, y_{2}, y_{3}\right\}$ such that $\left\{y_{3}, y_{4}, q_{1}, q_{2}\right\}$ is a circuit, as are $\left\{y_{2}, y_{4}, p_{1}, p_{3}\right\}$ and $\left\{y_{2}, y_{4}, p_{2}, p_{4}\right\}$. Indeed, it is easily seen that $\left\{y_{1}, y_{2}, y_{3}, y_{4}\right\}$ is a quad of $M$, and, in fact, that $P \cup\left\{y_{1}, y_{2}, y_{3}, y_{4}\right\}$ is a spiderlike 3 -separator. We have currently identified ten elements in the matroid $M$. By assumption there exists another element $\alpha \in Y$. Using the now familiar argument of Lemma 5.1.4 with respect to $\alpha$ and the element $p_{1} \in X$, we deduce the existence of a 4 -element circuit $C_{\alpha}$ where $\left\{\alpha, p_{1}, p_{3}\right\} \subseteq C_{\alpha}$. As $M$ contains no triangles, nor any restriction isomorphic to $U_{3,5}$, it follows that the fourth element $\beta$ of $C_{\alpha}$ is such that $\beta \notin X \cup\left\{y_{1}, y_{2}, y_{3}, y_{4}, \alpha\right\}$. However, by applying Lemma 5.1 .4 with respect to the elements $p_{1}$ and $y_{1}$, we can readily deduce that $C_{1}^{*}=\left\{p_{1}, p_{2}, y_{1}, y_{2}\right\}$ must be a cocircuit of $M$. But now $\left|C_{\alpha} \cap C_{1}^{*}\right|=1$ and we arrive at a contradiction. The matroid $M$ cannot be extended beyond its current construction which consists of ten points as is required by our hypothesis.

Suppose instead then that (iii) of Theorem 5.2.1 holds, giving $X$ as a spider-like 3-separator. Let $\{P, Q\}$ be the associated partition of $X$. The argument now proceeds precisely as in the partial spider-like case. We locate the appropriate circuits and cocircuits of $M$ using Lemma 5.1.4 and once again end up with a maximal-sized matroid which does not exceed twelve elements. We omit the details. In this case, a maximal matroid has exactly twelve elements. Once more, $Y$ is necessarily a quad, and the matroid $M$ we obtain is such that both $Y \cup P$ and $Y \cup Q$ are spider-like 3-separators. In the case that (i) of Theorem 5.2.1 holds, and that $X$ is a twisted 3-separator, one can once again readily produce an analogous argument. A maximal matroid is constructed that contains ten elements where $Y$ is again a quad attached to $X$ in such a manner as to give the appropriate circuits and cocircuits as necessitated by Lemma 5.1.4.

The only possibility that remains is that $X$ is a spike-like 3 -separator with associated partition $\left\{X_{1}, \ldots, X_{n}\right\}$. Again, using the strategy of implementing Lemma 5.1.4, we find that, as $M$ contains no triangles nor any $U_{3,5}$ restrictions, for each $X_{i} \in\left\{X_{1}, \ldots, X_{n}\right\}$, there exists a pair $\left\{y_{i}, y_{i}^{\prime}\right\}=Y_{i} \subseteq Y$ 
such that $X_{i} \cup Y_{i}$ is both a 4-element circuit, as well as a 4-element cocircuit. The matroid $M$ is thus a spike and our proof is complete.

Note that the maximal ten and twelve element matroids constructed in the non-spike cases in the proof of the above result all reduce to a very small class of matroids where the ground-set can be partitioned into an exact 3separation $(X, Y)$ where $Y$ is a quad and $X$ is either spider-like, partial spider-like, or twisted. See [10] for a complete survey of this collection of sporadic matroids. We are now ready to prove our main theorem.

Proof of Theorem 1.0.1. If the matroid $M$ contains a triangle or a triad, then by Theorem 2.1.1, the result holds. Otherwise, the theorem follows directly from Lemma 5.2.2. 


\section{Bibliography}

[1] Akkari, S., Oxley, J. G., Some local extremal connectivity results for matroids. Combin. Probab. Comput. 2 (1993) 367-384.

[2] Bixby, R. E., On Reid's characterization of the ternary matroids. J. Combin. Theory Ser. B 26 (1979) 174-204.

[3] Bixby, R. E., A simple theorem on 3-connectivity. Linear Algebra Appl. 45 (1982) 123-126.

[4] Clark, B., Fragility and excluded minors, PhD thesis, Victoria University of Wellington, 2014.

[5] Geelen, J. F., Gerards, A. M. H., and Kapoor, A., The excluded minors for GF(4)-representable matroids. J. Combin. Theory Ser. B 79 (2000) 247-299.

[6] Geelen, J. F., Gerards, A. M. H., and Whittle, G., Solving Rota's Conjecture. Notices Amer. Math. Soc. 61 736-743.

[7] Kahn, J., On the uniqueness of matroid representations over GF(4), Bull. London Math. Soc. 20 (1988) 5-10.

[8] Geelen, J. F., Whittle, G., Inequivalent representations of matroids over prime fields. Adv. in Appl. Math. 51 (2013) 1-175.

[9] Mayhew, D., Newman, M., Whittle, G., On excluded minors for realrepresentability. J. Combin. Theory Ser. B 99 (2009) 685-689. 
[10] Miller, J., Matroids in which every pair of elements belongs to both a 4circuit and a 4-cocircuit. MSc thesis, Victoria University of Wellington, 2014.

[11] Oxley, J. G., Matroid Theory, Second Edition, Oxford University Press, New York, 2011.

[12] Oxley, J. G., Semple, C., and Vertigan, D. L., Generalized Delta - Y exchanges and k-regular matroids J. Combin. Theory Ser. B 79 (2000) $1-65$.

[13] Oxley, J. G., Vertigan, D. L., and Whittle, G. P., On inequivalent representations of matroids over finite fields. J. Combin. Theory Ser. B 67 (1996) 325-343.

[14] Oxley, J. G., Wu, H., On the structure of 3-connected matroids and graphs, European J. Combin. 21 (2000), 667-688

[15] Rota, G. -C., Combinatorial theory, old and new. In Actes du Congrés International des Mathématiciens (Nice 1970), Tom 3, pp. 229-233. Gauthier-Villars, Paris (1971).

[16] Seymour, P. D., Matroid representation over GF(3). J. Combin. Theory Ser. B 26 (1979), 159-173.

[17] Seymour, P. D., Decomposition of regular matroids. J. Combin. Theory Ser. B 28 (1980), 305-359.

[18] Tutte, W. T., A homotopy theorem for matroids, I, II. Trans. Amer. Math. Soc. 88 (1958) 144-174.

[19] Tutte, W. T., Connectivity in matroids. Canad. J. Math. 18 (1966) 1301-1324.

[20] Vámos, P., On the representation of independence structures (unpublished manuscript). 
[21] Whitney, H., On the abstract properties of linear dependence. Amer. J. Math. 57 (1935), 509-533.

[22] Whittle, G. P., Stabilizers of classes of representable matroids. J. Combin. Theory Ser. B 77 (1999), 39-72.

[23] Whittle, G. P., Williams, A. D., On preserving matroid 3-connectivity relative to a fixed basis. European J. Combin. 34 (2013), 957-967. 